\title{
Triptycene-Based Luminescent Materials in Homoconjugated Charge Transfer Systems: Synthesis, Electronic Structures, AIE Activity and Highly Tunable Emissions
}

Puyi Lei,${ }^{\dagger}$ Songhe Zhang,${ }^{\dagger}$ Niu Zhang,${ }^{\dagger}$ Xiaodong Yin, ${ }^{\dagger}$ Nan Wang,${ }^{\dagger}$ and Pangkuan Chen ${ }^{*}, \dagger$

${ }^{\dagger}$ Beijing Key Laboratory of Photoelectronic/Electrophotonic Conversion Materials,

Key Laboratory of Cluster Science of the Ministry of Education, School of Chemistry and Chemical Engineering, Beijing Institute of Technology of China, Beijing, 102488, People's Republic of China. ^Analysis \& Testing Centre, Beijing Institute of Technology, 102488, People’s Republic of China. 


\section{Experimental Section}

Materials and General Methods. $n$-Butyllithium, phosphinic acid, potassium carbonate, pyridine, $o$-phenylendiamine, $o$-dichlorobenzene, cuprous iodide, trifluoroacetic anhydride, 18-crown-6, carbazole, phenothiazine, diisopropylethylamine, sodium $t$-butoxide, xantphos and tribenzylidene acetone dipalladium(II) were purchased from Energy Chemical. Tetrahydrofuran, dichloromethane, petroleum ether, methanol and ethyl acetate were purchased from Sinopharm Chemical Reagent Co., Ltd. Chemicals were used without further purification unless otherwise noted. Anhydrous solvents were distilled from commercial sources with sodium/benzopheneone or calcium hydride.

All commercial chemicals were used without further purification. ${ }^{1} \mathrm{H}$ NMR spectra were recorded on a $400 \mathrm{MHz}$ Bruker spectrometer. ${ }^{13} \mathrm{C}$ NMR spectra were recorded on the 101 or $176 \mathrm{MHz}$ Bruker spectrometers. ${ }^{11} \mathrm{~B}$ NMR spectra were recorded on a 128 $\mathrm{MHz}$ Bruker spectrometer. ${ }^{11} \mathrm{~B}$ NMR spectra were acquired with boron-free quartz NMR tubes and the spectra were referenced externally to $\mathrm{BF}_{3} \cdot \mathrm{Et}_{2} \mathrm{O}(\delta=0)$. High resolution mass spectral data were obtained via ESI on an Agilent (Q-TOF 6520) analyzer.

UV-visible absorption spectra were recorded on a Cary 300 UV-Vis spectrophotometer. Luminescent spectra were recorded on an Edinburgh Instrument FLS980 or Lengguang Tech F97PrO spectrophotometer. Fluorescent quantum efficiencies were determined using a Hamamatsu C11347-11 Quantaurus-QY spectrometer.

X-ray single crystals were obtained on a Bruker D8 X-ray single crystal Venture diffractometer. SAINT5.0 and SADABS programs are used for the reduction and absorption correction of crystal data. The resolution and refinement of the crystal structure are obtained on the SHELXTL-97 software. Using the direct or Patterson methods, all nonhydrogen source coordinates are obtained by using the differential Fourier method and the least square method. Then the geometric method and the difference value are used. The hydrogen atom coordinates were obtained by Fourier method, and the crystal structure was obtained. The CCDC numbers of 2011196 (CzCz), and 2011197 (Pz-Pz) are deposited.

DFT calculations were performed with the Gaussian 09 program. Geometries and electronic properties were calculated by means of hybrid density functional B3LYP and CAM-B3LYP with the basis set of 6-31G*. The input files and orbital representations were generated with Gaussview 5.0 (scaling radii of $75 \%$, isovalue $=0.02$ ). Excitation data were calculated using TD-DFT (B3LYP/6-31G* and B3LYP/6-311G**). The true minimum of resulting structures was confirmed to be stationary points through vibrational frequency analysis with absorbance of imaginary frequencies. 


\section{Synthetic Procedures}

\subsection{Synthesis of 1}<smiles>Nc1ccc2c(c1)C(=O)C1C=CNC=C1C2=O</smiles>

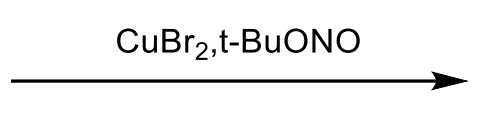<smiles>O=C1c2ccc(Br)cc2C(=O)C2C=C(Br)C=CC12</smiles>

Compound 1 was synthesized via the previously reported procedure. ${ }^{1}$

\subsection{Synthesis of 2}<smiles>O=C1C2=CC(Br)=CC(=O)C2C(=O)c2cc(Br)ccc21</smiles>

1

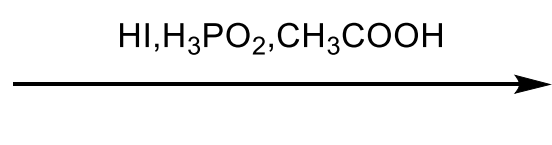

Compound 2 was synthesized via the previously reported procedure. ${ }^{2}$

\subsection{Synthesis of 3}<smiles>Brc1ccc2cc3ccc(Br)cc3cc2c1</smiles>

2

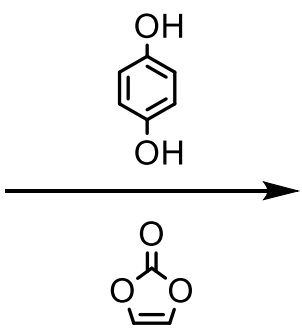

2<smiles>Brc1ccc2cc3ccc(Br)cc3cc2c1</smiles>

A mixture of 2 (600 mg, $1.79 \mathrm{mmol}$ ), hydroquinone (10 mg, 5\%), vinylene carbonate $(2 \mathrm{~mL}, 31.6 \mathrm{mmol})$ in anhydrous xylene $(5 \mathrm{~mL})$ was added into a pressure vessel, degassed and heated at $190{ }^{\circ} \mathrm{C}$ for $3 \mathrm{~d}$. The reaction was concentrated and purified by column chromatography on silica gel (petroleum ether /ethyl acetate $=10: 1, \mathrm{v} / \mathrm{v}$ ) to give 3 as a white solid (324.3 mg, $44 \%) .{ }^{1} \mathrm{H}$ NMR (400 MHz, Methanol- $\left.d_{4}\right) \delta$ 7.65-7.57 (m, 2H), $7.42(\mathrm{ddd}, J=7.9,4.8,1.9 \mathrm{~Hz}, 2 \mathrm{H}), 7.34(\mathrm{dd}, J=11.4,8.0 \mathrm{~Hz}, 2 \mathrm{H}), 4.97$ (q, $J=$ $2.1 \mathrm{~Hz}, 2 \mathrm{H}), 4.81(\mathrm{q}, J=1.9 \mathrm{~Hz}, 2 \mathrm{H}) \cdot{ }^{13} \mathrm{C} \mathrm{NMR}\left(101 \mathrm{MHz}, \mathrm{CDCl}_{3}\right) \delta 140.1,139.3$, $136.7,135.8,130.5,130.2,129.3,128.6,128.1,127.2,121.0,120.8,76.1$. 


\subsection{Synthesis of 4}

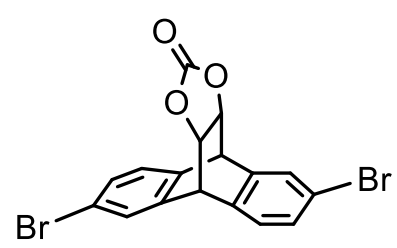

3

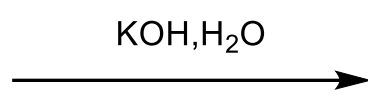

4

To a solution of $3(1.689 \mathrm{~g}, 4 \mathrm{mmol})$ in ethanol $(15 \mathrm{~mL})$ was added dropwise a aqueous solution of $\mathrm{KOH}(825 \mathrm{mg}, 12 \mathrm{mmol}, 9 \mathrm{~mL})$. The reaction was stirred at $100{ }^{\circ} \mathrm{C}$ overnight. After the reaction was complete, it was cooled to r.t, and extracted with $\mathrm{CH}_{2} \mathrm{Cl}_{2}(3 \times 30 \mathrm{~mL})$ and washed with water $(3 \times 30 \mathrm{~mL})$. The combined organic layer was dried over anhydrous sodium sulfate. The crude product was filtered under reduced vacuum to afford 4 as a maroon solid $(1.428 \mathrm{~g}, 90 \%)$ without further purification. ${ }^{1} \mathrm{H}$ NMR (400 MHz, $\left.\mathrm{CDCl}_{3}\right) \delta 7.38(\mathrm{dd}, J=12.9,1.8 \mathrm{~Hz}, 2 \mathrm{H}), 7.20(\mathrm{dt}, J=7.9,1.4 \mathrm{~Hz}$, $2 \mathrm{H}), 7.17-7.08(\mathrm{~m}, 2 \mathrm{H}), 4.23(\mathrm{~d}, J=8.5 \mathrm{~Hz}, 2 \mathrm{H}), 3.93-3.74(\mathrm{~m}, 2 \mathrm{H})$.

\subsection{Synthesis of 5}

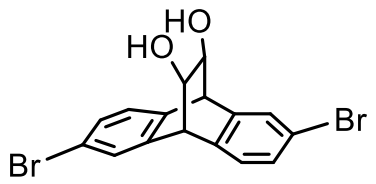

4

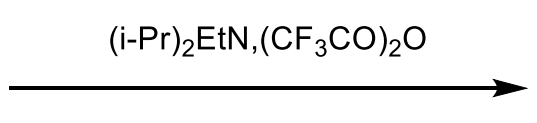

A mixture of dry $\mathrm{CH}_{2} \mathrm{Cl}_{2}(90 \mathrm{~mL})$, DMSO (40 mL) was degassed, cooled to $-60{ }^{\circ} \mathrm{C}$ and kept stirring for 20 min under $\mathrm{N}_{2}$, and then anhydrous trifluoroacetic anhydride (40 $\mathrm{mL}$ ) was added dropwise. After cooled for $1 \mathrm{~h}$, a solution of 4 (7.690 g, $19.4 \mathrm{mmol})$ dissolved in dry $\mathrm{CH}_{2} \mathrm{Cl}_{2}(90 \mathrm{~mL})$ and DMSO $(40 \mathrm{~mL})$ was added dropwise. After cooled for another hour, $\mathrm{N}$-ethyldiisopropylamine $(100 \mathrm{~mL})$ was added dropwise. After cooled for $1 \mathrm{~h}$, the reaction mixture was allowed to warm up to r.t. and kept stirring overnight. The reaction was then quenched by hydrochloric acid $(100 \mathrm{~mL}, 1 \mathrm{M})$ and extracted with $\mathrm{CH}_{2} \mathrm{Cl}_{2}(3 \times 30 \mathrm{~mL})$ and washed with brine and water. The combined organic layer was dried over anhydrous sodium sulfate. The crude product was purified by column chromatography on silica gel (petroleum ether/dichloromethane $=1: 1$, v/v) to give 5 as an orange solid $(5.77 \mathrm{~g}, 75 \%) .{ }^{1} \mathrm{H}$ NMR $\left(400 \mathrm{MHz}, \mathrm{CDCl}_{3}\right) \delta 7.63(\mathrm{~d}, J=1.8 \mathrm{~Hz}, 2 \mathrm{H})$, $7.51(\mathrm{~d}, J=1.9 \mathrm{~Hz}, 2 \mathrm{H}), 7.34(\mathrm{~d}, J=8.0 \mathrm{~Hz}, 2 \mathrm{H}), 4.93(\mathrm{~s}, 2 \mathrm{H})$. 


\subsection{Synthesis of 6}

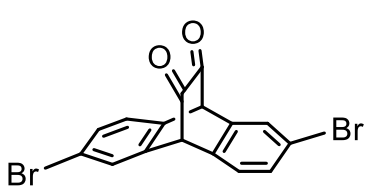

5
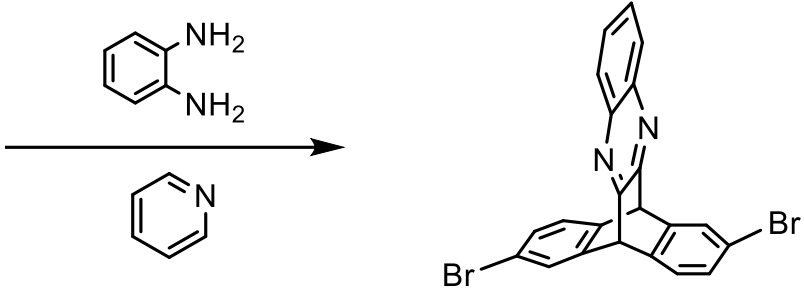

6

To a solution of $o$-phenylenediamine $(1.298 \mathrm{~g}, 12 \mathrm{mmol})$ in anhydrous methanol $(60 \mathrm{~mL})$ and pyridine $(12.9 \mathrm{~mL})$ was added dropwise a solution of 5 (4.745 g, $12 \mathrm{mmol})$ in dry methanol $(30 \mathrm{~mL})$. The reaction was stirred at $65{ }^{\circ} \mathrm{C}$ overnight under $\mathrm{N}_{2}$. After the reaction was complete, it was cooled to r.t, filtered under reduced vacuum and washed with methanol to afford 6 as a pale solid $(3.31 \mathrm{~g}, 59 \%) .{ }^{1} \mathrm{H}$ NMR $(400 \mathrm{MHz}$, $\left.\mathrm{CDCl}_{3}\right) \delta 8.00-7.82(\mathrm{~m}, 2 \mathrm{H}), 7.67(\mathrm{~d}, J=3.0 \mathrm{~Hz}, 4 \mathrm{H}), 7.54-7.35(\mathrm{~m}, 2 \mathrm{H}), 7.31-7.27$ $(\mathrm{m}, 2 \mathrm{H}), 5.60-5.52(\mathrm{~m}, 2 \mathrm{H})$.

\subsection{Synthesis of $\mathrm{Cz}-\mathrm{Br}$}

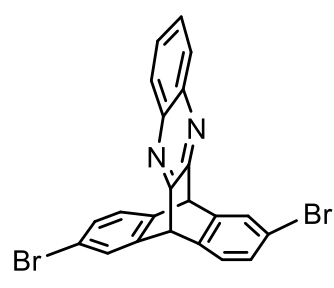

6

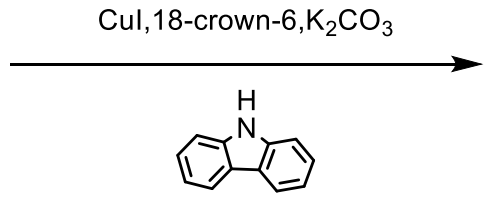

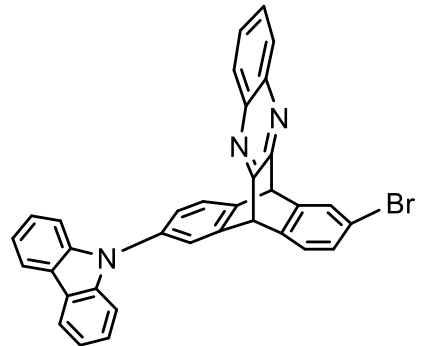

$\mathrm{Cz}-\mathrm{Br}$

6 (1.0 g, 2.15mmol), carbazole (400 mg, 1.1 equiv), anhydrous $\mathrm{K}_{2} \mathrm{CO}_{3}(595 \mathrm{mg}, 2$ equiv), cuprous iodide (20 mg, 5\%) and 18-crown-6 (57 mg, 10\%) were dissolved in dry $o$-dichlorobenzene $(15 \mathrm{~mL})$. The mixture was degassed and was then heated to $180{ }^{\circ} \mathrm{C}$ and refluxed overnight under $\mathrm{N}_{2}$. After the reaction was complete, the reaction was cooled to r.t, concentrated and purified by column chromatography on silica gel (petroleum ether /ethyl acetate $=15: 1, \mathrm{v} / \mathrm{v}$ ) to give $\mathbf{C z - B r}$ as a pale solid $(723 \mathrm{mg}, 61 \%)$. ${ }^{1} \mathrm{H}$ NMR $\left(400 \mathrm{MHz}, \mathrm{CDCl}_{3}\right) \delta 8.11(\mathrm{~d}, J=7.7 \mathrm{~Hz}, 2 \mathrm{H}), 7.98(\mathrm{tt}, J=7.0,3.5 \mathrm{~Hz}, 2 \mathrm{H})$, 7.80-7.69 (m, 5H), 7.46 (d, $J=7.9 \mathrm{~Hz}, 1 \mathrm{H}), 7.39-7.26(\mathrm{~m}, 8 \mathrm{H}), 5.71(\mathrm{~d}, J=24.0,2 \mathrm{H})$. ${ }^{13} \mathrm{C}$ NMR $\left(101 \mathrm{MHz}, \mathrm{CDCl}_{3}\right) \delta 156.3,144.1,143.6,140.8,140.5,139.5,139.4,136.5$, $130.3,129.9,129.7,128.7,126.7,126.4,126.0,125.7,123.8,123.4,120.6,120.3,120.1$, 109.7, 54.7. ESI-HRMS (m/z): calcd. for $\mathrm{C}_{34} \mathrm{H}_{20} \mathrm{BrN}_{3}[\mathrm{M}+\mathrm{H}]^{+}$550.0913, found 550.0898 . 


\subsection{Synthesis of Pz-Br}

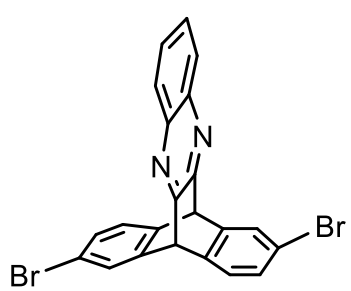

6

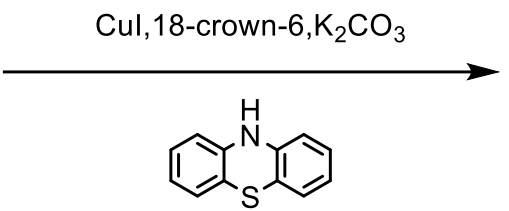

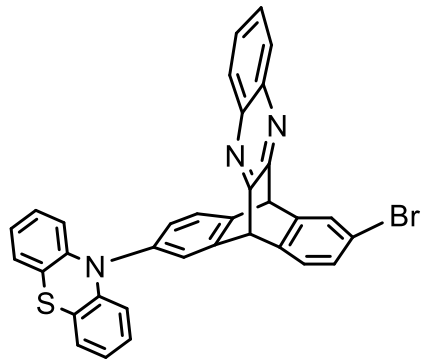

$\mathrm{Pz}-\mathrm{Br}$

6 (1.0 g, $2.15 \mathrm{mmol}$ ), phenothiazine (471 mg, 1.1 equiv), anhydrous $\mathrm{K}_{2} \mathrm{CO}_{3}$ (595 $\mathrm{mg}, 2$ equiv), cuprous iodide (20 mg, 5\%) and 18-crown-6 (57 mg, 10\%) were dissolved in dry o-dichlorobenzene $(15 \mathrm{~mL})$. The mixture was degassed, heated to $180{ }^{\circ} \mathrm{C}$ and refluxed overnight under $\mathrm{N}_{2}$ in dark. After the reaction was complete, the reaction was cooled to r.t, concentrated and purified by column chromatography on silica gel (petroleum ether /ethyl acetate $=15: 1, \mathrm{v} / \mathrm{v}$ ) to give $\mathbf{P z}-\mathbf{B r}$ as a yellowish solid (556 mg, 44\%). ${ }^{1} \mathrm{H}$ NMR (400 MHz, $\left.\mathrm{CDCl}_{3}\right) \delta 7.96$ (ddd, $J=10.6,7.0,3.8 \mathrm{~Hz}, 2 \mathrm{H}$ ), $7.74(\mathrm{~d}, J=1.8 \mathrm{~Hz}, 1 \mathrm{H}), 7.72-7.68(\mathrm{~m}, 3 \mathrm{H}), 7.55(\mathrm{~d}, J=1.9 \mathrm{~Hz}, 1 \mathrm{H}), 7.43(\mathrm{~d}, J=7.9$ $\mathrm{Hz}, 1 \mathrm{H}), 7.31$ (dd, $J=7.9,1.9 \mathrm{~Hz}, 1 \mathrm{H}), 7.18(\mathrm{dd}, J=7.9,2.0 \mathrm{~Hz}, 1 \mathrm{H}), 7.07-6.98(\mathrm{~m}$, 2H), 6.86-6.73 (m, 4H), 6.21-6.11 (m, 2H), $5.69(\mathrm{~s}, 1 \mathrm{H}), 5.64(\mathrm{~s}, 1 \mathrm{H})$. ESI-HRMS $(\mathrm{m} / \mathrm{z})$ : calcd. for $\mathrm{C}_{34} \mathrm{H}_{20} \mathrm{BrN}_{3} \mathrm{~S}[\mathrm{M}+\mathrm{H}]^{+} 582.0634$, found 582.0643 .

\subsection{Synthesis of $\mathrm{Mes}_{2} \mathrm{BF}$}

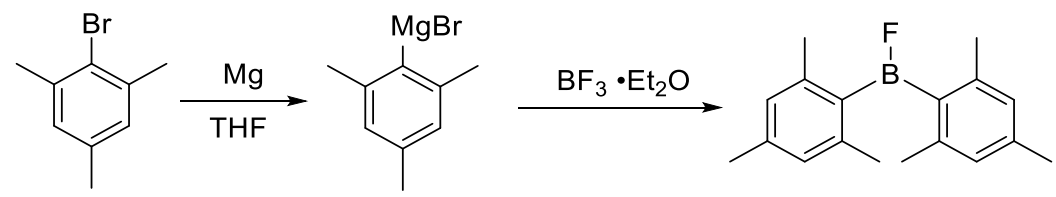

Compound Mes2BF was synthesized via the previously reported procedure. ${ }^{3}$

\subsection{Synthesis of 7}<smiles>Brc1ccc(Br)cc1</smiles><smiles>Cc1ccc(Br)cc1</smiles>

7

Compound 7 was synthesized via the previously reported procedure. ${ }^{4}$ 


\subsection{Synthesis of 8}

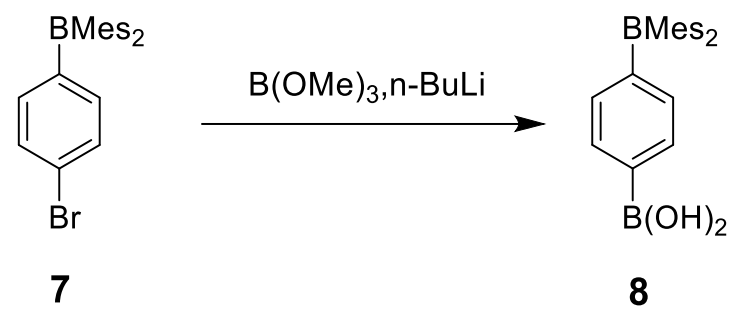

Compound $\mathbf{8}$ was synthesized via the previously reported procedure. ${ }^{5}$

\subsection{Synthesis of Cz-BMes}

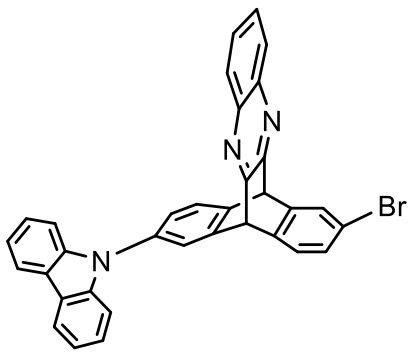

$\mathrm{Cz}-\mathrm{Br}$

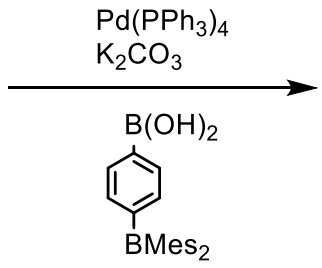

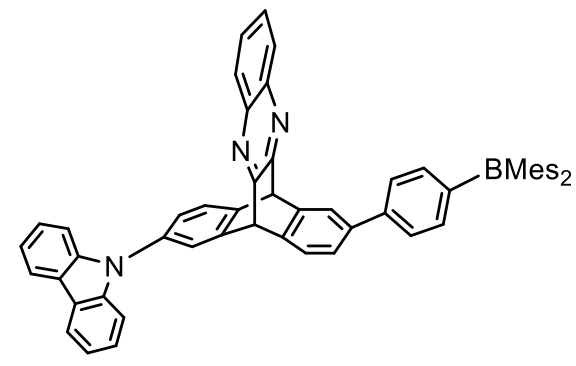

Cz-BMes

Cz-Br (55 mg, $0.1 \mathrm{mmol}$ ), tetrakis(triphenylphosphine)palladium(0) (18.5 mg, 5\%) and 8 (45 mg, 1.2 equiv) was dissolved in toluene $(6 \mathrm{~mL})$. The mixture was degassed, then a solution of $\mathrm{K}_{2} \mathrm{CO}_{3}$ ( $83 \mathrm{mg}, 6$ equiv) dissolved in water $(3 \mathrm{~mL})$ was added dropwise under $\mathrm{N}_{2}$. The mixture was then heated to $110{ }^{\circ} \mathrm{C}$ and refluxed overnight under $\mathrm{N}_{2}$. After the reaction was complete, the reaction was cooled to r.t, filtrated, concentrated and purified by column chromatography on silica gel (petroleum ether /ethyl acetate $=15: 1, \mathrm{v} / \mathrm{v}$ ) to give $\mathbf{C z}-\mathbf{B M e s}$ as a pale solid (23 mg, 29\%). Melting point: $470 \mathrm{~K} .{ }^{1} \mathrm{H}$ NMR $\left(400 \mathrm{MHz}, \mathrm{CDCl}_{3}\right) \delta 8.11(\mathrm{~d}, J=7.7 \mathrm{~Hz}, 2 \mathrm{H}), 7.99(\mathrm{dq}, J=11.0,3.7$ $\mathrm{Hz}, 2 \mathrm{H}), 7.90(\mathrm{~d}, J=1.5 \mathrm{~Hz}, 1 \mathrm{H}), 7.79-7.74(\mathrm{~m}, 2 \mathrm{H}), 7.72-7.64(\mathrm{~m}, 3 \mathrm{H}), 7.59-7.52(\mathrm{~m}$, 4H), 7.47 (dd, $J=7.7,1.7 \mathrm{~Hz}, 1 \mathrm{H}), 7.39-7.23(\mathrm{~m}, 7 \mathrm{H}), 7.24(\mathrm{~d}, J=1.4 \mathrm{~Hz}, 1 \mathrm{H}), 6.83$ $(\mathrm{s}, 4 \mathrm{H}), 5.84(\mathrm{~s}, 1 \mathrm{H}), 5.75(\mathrm{~s}, 1 \mathrm{H}), 2.31(\mathrm{~s}, 6 \mathrm{H}), 2.02(\mathrm{~s}, 12 \mathrm{H}) .{ }^{13} \mathrm{C}$ NMR $(101 \mathrm{MHz}$, $\left.\mathrm{CDCl}_{3}\right) \delta 141.1,140.8,138.7,137.0,129.5,128.7,128.2,126.7,125.9,125.5,123.8$, 123.4, 120.3, 120.1, 109.8, 23.5, 21.2. ${ }^{11} \mathrm{~B}$ NMR $\left(225 \mathrm{MHz}, \mathrm{CDCl}_{3}\right) \delta 28$. ESI-HRMS $(\mathrm{m} / \mathrm{z})$ : calcd. for $\mathrm{C}_{58} \mathrm{H}_{46} \mathrm{BN}_{3}[\mathrm{M}+\mathrm{H}]^{+} 796.3858$, found 796.3847 . 


\subsection{Synthesis of Pz-BMes}

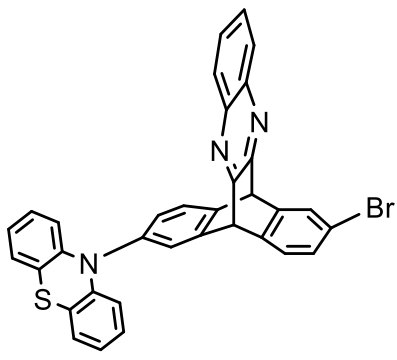

$\mathrm{Pz}-\mathrm{Br}$

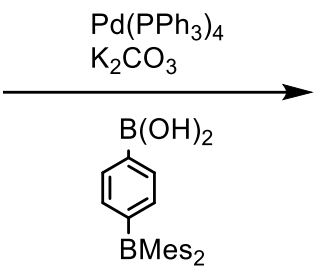

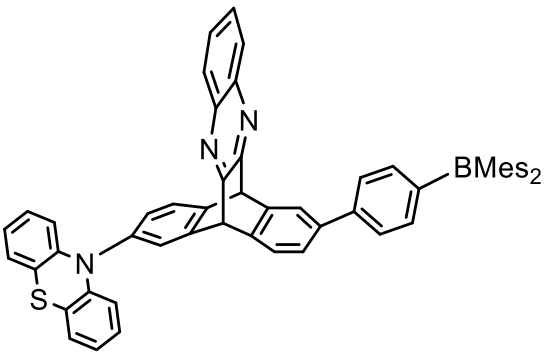

Pz-BMes

A similar procedure for $\mathbf{C z}$-BMes was performed to give Pz-BMes as an yellowish solid (19 mg, 28\%). Melting point: $\left.466 \mathrm{~K} .{ }^{1} \mathrm{H} \mathrm{NMR} \mathrm{(400} \mathrm{MHz,} \mathrm{CDCl}_{3}\right) \delta 7.97(\mathrm{td}, J=$ 7.2, 3.6 Hz, 2H), $7.87(\mathrm{~d}, J=1.6 \mathrm{~Hz}, 1 \mathrm{H}), 7.77-7.59(\mathrm{~m}, 4 \mathrm{H}), 7.59-7.51(\mathrm{~m}, 5 \mathrm{H}), 7.46$ $(\mathrm{dd}, J=7.6,1.8 \mathrm{~Hz}, 1 \mathrm{H}), 7.18(\mathrm{dd}, J=7.8,2.0 \mathrm{~Hz}, 1 \mathrm{H}), 7.01(\mathrm{dd}, J=4.2,2.6 \mathrm{~Hz}, 2 \mathrm{H})$, 6.90-6.70 (m, H), 6.23-6.11 (m, 2H), $5.75(\mathrm{~d}, J=35.0 \mathrm{~Hz}, 2 \mathrm{H}), 2.31(\mathrm{~s}, 6 \mathrm{H}), 2.01(\mathrm{~s}$, 12H). ${ }^{13} \mathrm{C} \mathrm{NMR}\left(101 \mathrm{MHz}, \mathrm{CDCl}_{3}\right) \delta 139.8,137.7,136.0,129.2,128.5,127.6,127.2$, 125.8, 125.6, 124.9, 121.7, 115.8, 76.2, 28.7, 22.4, 21.7, 20.2, 13.1. ${ }^{11}$ B NMR (128 $\left.\mathrm{MHz} \mathrm{CDCl}_{3}\right) \delta 26$. ESI-HRMS (m/z): calcd. for $\mathrm{C}_{58} \mathrm{H}_{46} \mathrm{BN}_{3} \mathrm{~S}[\mathrm{M}+\mathrm{H}]^{+} 828.3578$, found 828.3580 .

\subsection{Synthesis of $\mathrm{Cz}-\mathrm{Cz}$}

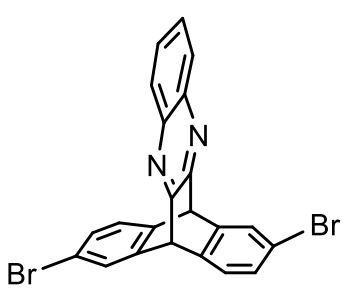

6

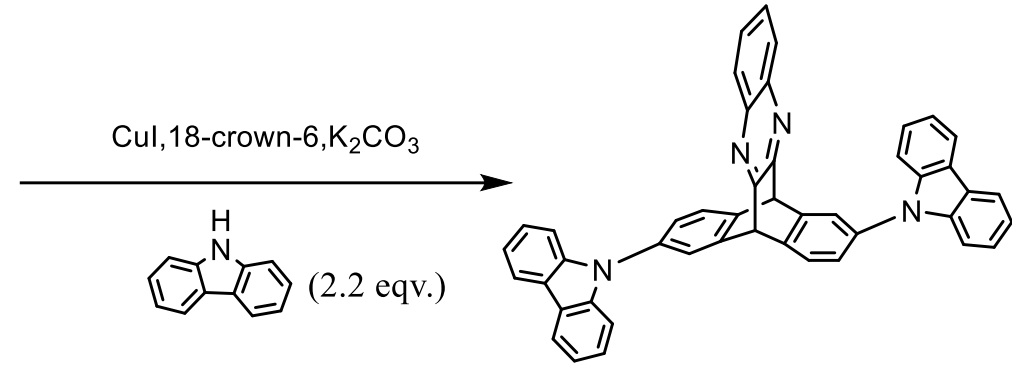

$\mathrm{Cz}-\mathrm{Cz}$

A similar procedure for $\mathbf{C z - B r}$ was performed to give $\mathbf{C z}-\mathbf{C z}$ as a yellowish solid (14 mg, 9\%). Melting point: $571 \mathrm{~K} .{ }^{1} \mathrm{H} \mathrm{NMR}\left(400 \mathrm{MHz}, \mathrm{CDCl}_{3}\right) \delta 8.13(\mathrm{~d}, J=7.6 \mathrm{~Hz}$, $4 \mathrm{H}), 8.03(\mathrm{dd}, J=6.2,3.2 \mathrm{~Hz}, 2 \mathrm{H}), 7.85-7.77(\mathrm{~m}, 4 \mathrm{H}), 7.73(\mathrm{dd}, J=6.2,3.4 \mathrm{~Hz}, 2 \mathrm{H})$, 7.49-7.32 (m, 10H), 7.29 (dd, $J=7.8,4.0 \mathrm{~Hz}, 4 \mathrm{H}), 5.84(\mathrm{~s}, 2 \mathrm{H}) .{ }^{13} \mathrm{C}$ NMR $(101 \mathrm{MHz}$, $\left.\mathrm{CDCl}_{3}\right) \delta 156.6,143.8,140.8,140.8,139.5,136.5,129.7,128.7,126.5,126.0,125.6$, $123.9,123.4,120.4,120.2,109.8,55.1$. ESI-HRMS (m/z): calcd. for $\mathrm{C}_{46} \mathrm{H}_{28} \mathrm{~N}_{4}[\mathrm{M}+\mathrm{H}]^{+}$ 637.2387, found 637.2372. 


\subsection{Synthesis of Pz-Pz}

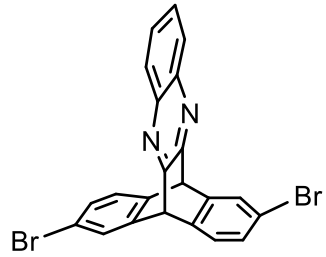

6
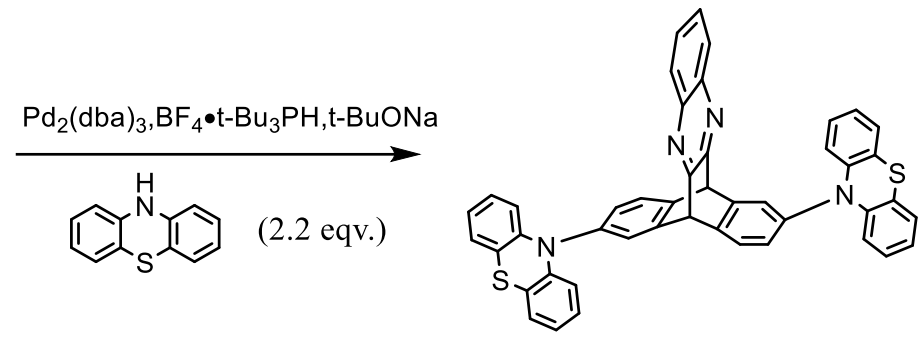

Pz-Pz

6 (46.4 mg, $0.1 \mathrm{mmol}$ ), phenothiazine (43.8 mg, 2.2 equiv), $t$-BuONa (29 mg, 3 equiv), tris(dibenzylidineacetone)dipalladium(0) (4.6 $\mathrm{mg}, 5 \%)$ and tri-tertbutylphosphonium tetrafluoroborate $(3 \mathrm{mg}, 10 \%)$ were dissolved in dry toluene $(6 \mathrm{~mL})$. The mixture was degassed, heated to $107{ }^{\circ} \mathrm{C}$ and refluxed $20 \mathrm{~h}$ under $\mathrm{N}_{2}$. After the reaction was complete, the reaction was cooled to r.t, filtrated, concentrated and purified by column chromatography on silica gel (petroleum ether/ethyl acetate $=10: 1$, v/v first; then dichloromethane) to give Pz-Pz as a brown solid (22 mg, 29\%). Melting point: $558 \mathrm{~K} .{ }^{1} \mathrm{H} \mathrm{NMR}\left(400 \mathrm{MHz}, \mathrm{CDCl}_{3}\right) \delta 7.98(\mathrm{dd}, J=6.2,3.4 \mathrm{~Hz}, 2 \mathrm{H}), 7.71(\mathrm{dd}, J$ $=6.8,3.8 \mathrm{~Hz}, 4 \mathrm{H}), 7.58(\mathrm{~d}, J=1.6 \mathrm{~Hz}, 2 \mathrm{H}), 7.18(\mathrm{dd}, J=8.0,2.0 \mathrm{~Hz}, 2 \mathrm{H}), 7.12-7.02$ $(\mathrm{m}, 4 \mathrm{H}), 6.85(\mathrm{dt}, J=5.8,2.4 \mathrm{~Hz}, 8 \mathrm{H}), 6.37-6.24(\mathrm{~m}, 4 \mathrm{H}), 5.72(\mathrm{~s}, 2 \mathrm{H}) .{ }^{13} \mathrm{C}$ NMR $(101$ $\left.\mathrm{MHz}, \mathrm{CDCl}_{3}\right) \delta 156.8,144.5,143.8,140.6,140.4,139.4,129.6,128.7,127.7,127.1$, 127.0, 126.9, 125.7, 123.1, 122.4, 117.6, 55.0. ESI-HRMS (m/z): calcd. for $\mathrm{C}_{46} \mathrm{H}_{28} \mathrm{~N}_{4} \mathrm{~S}_{2}$ $[\mathrm{M}+\mathrm{H}]^{+} 701.1828$, found 701.1821 .

\subsection{Synthesis of Cz-BPI}

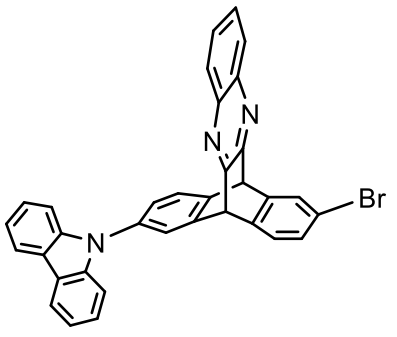

$\mathrm{Cz}-\mathrm{Br}$

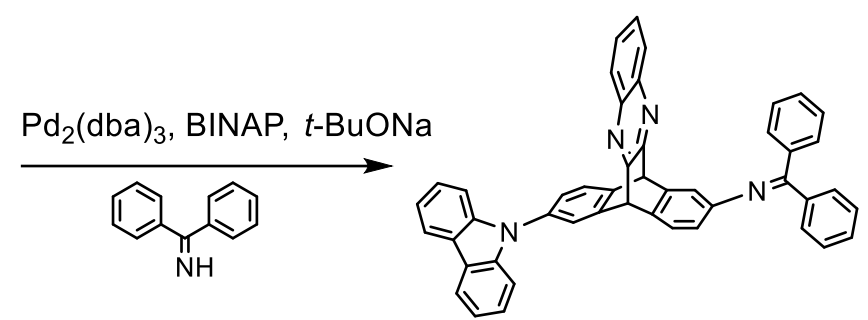

Cz-BPI

Cz-Br (55 mg, $0.1 \mathrm{mmol}$ ), benzophenonimine (20 mg, $18.5 \mu \mathrm{L}, 1.1$ equiv), $t$-BuONa (20 mg, 2.0 equiv), tris(dibenzylidineacetone)dipalladium(0) (4.6 mg, 5\%) and BINAP(bis(diphenulphosphino-1,1'-binaphthalene, $10 \mathrm{mg}, 15 \%$ ) were dissolved in dry toluene $(6 \mathrm{~mL})$. The mixture was degassed, heated to $107^{\circ} \mathrm{C}$ and refluxed $40 \mathrm{~h}$ under $\mathrm{N}_{2}$. After the reaction was complete, the reaction was cooled to r.t, filtrated, washed with $\mathrm{CH}_{2} \mathrm{Cl}_{2}$, brine and water. The combined organic layer was dried over anhydrous sodium sulfate, concentrated and purified by column chromatography on silica gel (petroleum ether/ethyl acetate $=15: 1, \mathrm{v} / \mathrm{v}$ ) to give Cz-BPI as a yellow solid (57 mg, 
88\%). ${ }^{1} \mathrm{H}$ NMR (400 MHz, $d_{6}$-DMSO) $\delta 8.23(\mathrm{~d}, J=7.7 \mathrm{~Hz}, 2 \mathrm{H}), 8.03-7.93(\mathrm{~m}, 2 \mathrm{H})$, 7.90 (dd, $J=4.9,2.9 \mathrm{~Hz}, 2 \mathrm{H}), 7.83-7.75(\mathrm{~m}, 2 \mathrm{H}), 7.65-7.59(\mathrm{~m}, 2 \mathrm{H}), 7.57-7.50$ (m, 1H), 7.50-7.34 (m, 6H), 7.33-7.18 (m, 8H), 7.14 (dd, $J=7.3,2.0 \mathrm{~Hz}, 2 \mathrm{H}), 6.46$ (dd, $J$ $=7.9,2.0 \mathrm{~Hz}, 1 \mathrm{H}), 5.89(\mathrm{~s}, 2 \mathrm{H}) .{ }^{13} \mathrm{C} \mathrm{NMR}\left(101 \mathrm{MHz}, d_{6}\right.$-DMSO) $\delta 168.2,157.5,150.1$, 144.9, 140.6, 139.2, 139.1, 136.6, 136.1, 135.6, 133.2, 131.5, 130.1, 129.2, 128.9, 129.1, $128.4,127.0,126.7,125.8,125.3,123.6,123.2$, 120.9, 120.6, 118.7, 110.2, 65.5, 55.4, $54.0,53.4,29.5$.

\subsection{Synthesis of $\mathrm{Cz}-\mathrm{NH}_{2}$}

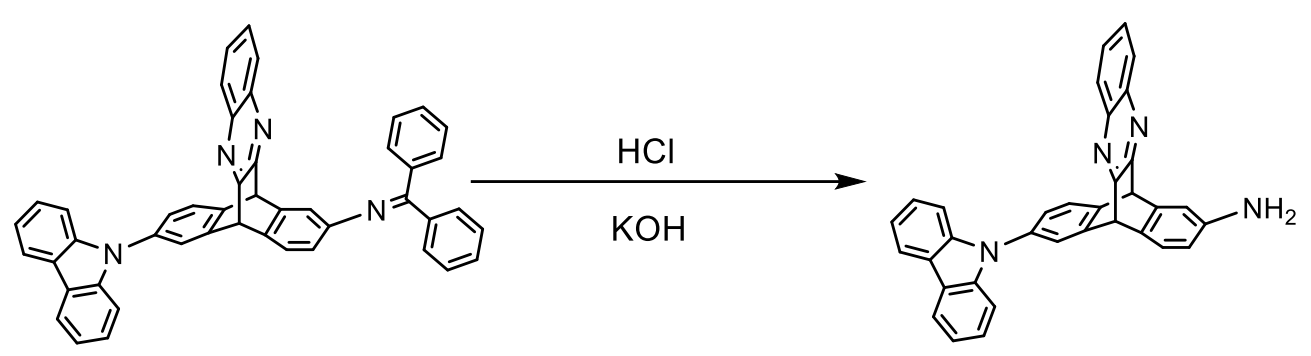

Cz-BPI

$\mathrm{Cz}-\mathrm{NH}_{2}$

Cz-BPI (57 mg, $0.088 \mathrm{mmol}$ ) was dissolved in 1M HCl/THF solution (1:1, v/v, 5 $\mathrm{mL}$ ) and stirred at room temperature for $4 \mathrm{~h}$. THF was removed under reduced pressure and the aqueous layer was basified to a $\mathrm{pH}=10-14$ using a solution of $\mathrm{KOH}(1 \mathrm{M})$. The aqueous layer was extracted with $\mathrm{CH}_{2} \mathrm{Cl}_{2}$ there times, and the combined organic layer was dried over anhydrous sodium sulfate, concentrated and purified by column chromatography on silica gel (dichloromethane, then methanol) to give $\mathbf{C z}-\mathbf{N H}_{\mathbf{2}}$ as a yellow solid (33 mg, 77\%). ${ }^{1} \mathrm{H}$ NMR $\left(400 \mathrm{MHz}, \mathrm{CDCl}_{3}\right) \delta 8.04(\mathrm{~d}, J=7.7 \mathrm{~Hz}, 2 \mathrm{H})$, $7.88(\mathrm{tt}, J=6.7,3.3 \mathrm{~Hz}, 2 \mathrm{H}), 7.71-7.53(\mathrm{~m}, 4 \mathrm{H}), 7.33-7.20(\mathrm{~m}, 7 \mathrm{H}), 7.16(\mathrm{~s}, 1 \mathrm{H}), 6.90$ $(\mathrm{d}, J=2.2 \mathrm{~Hz}, 1 \mathrm{H}), 6.39$ (dd, $J=8.0,2.3 \mathrm{~Hz}, 1 \mathrm{H}), 5.53(\mathrm{~d}, J=16.6 \mathrm{~Hz}, 2 \mathrm{H}), 3.63(\mathrm{~m}$, $2 \mathrm{H})$. ESI-HRMS (m/z): calcd. for $\mathrm{C}_{34} \mathrm{H}_{22} \mathrm{~N}_{4}[\mathrm{M}+\mathrm{H}]^{+} 487.1917$, found 487.1920.

\subsection{Synthesis of Cz-Npa}

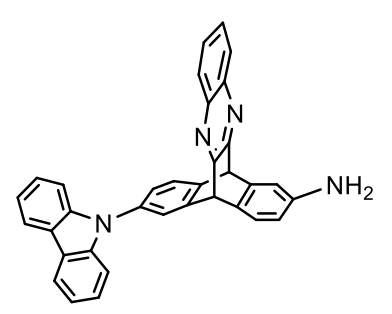

$\mathrm{Cz}-\mathrm{NH}_{2}$

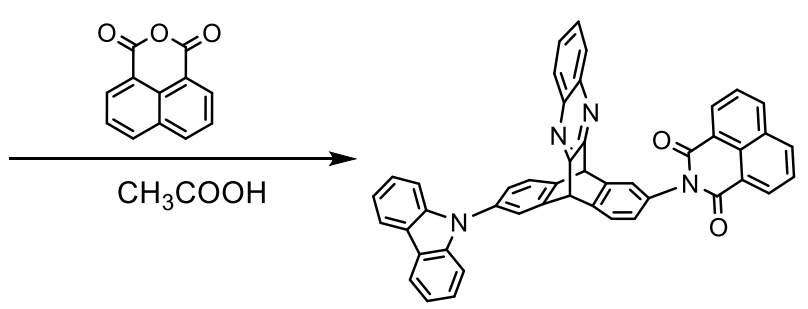

Cz-Npa

Cz-NH2 (91 mg, $0.187 \mathrm{mmol}$ ), 1,8-naphthalic anhydride (37 mg, $0.187 \mathrm{mmol}, 1$ equiv) was dissolved in acetic acid $(5 \mathrm{~mL})$ and refluxed for $5 \mathrm{~h}$. The reaction was cooled to r.t. and $10 \mathrm{~mL}$ water was added. The precipitates were recovered by filtration, and the crude product was dried under vacuo and purified by column chromatography on 
silica gel (dichloromethane, then methanol) to obtain Cz-Npa as a pale solid (66 mg, $53 \%)$. Melting point: $483 \mathrm{~K} .{ }^{1} \mathrm{H}$ NMR $\left(400 \mathrm{MHz}, \mathrm{CDCl}_{3}\right) \delta 8.62(\mathrm{dd}, J=7.2,1.0 \mathrm{~Hz}$, $2 \mathrm{H}), 8.30(\mathrm{dd}, J=8.2,0.8 \mathrm{~Hz}, 2 \mathrm{H}), 8.10(\mathrm{~d}, J=7.6 \mathrm{~Hz}, 2 \mathrm{H}), 8.02-7.93(\mathrm{~m}, 2 \mathrm{H}), 7.82$ $(\mathrm{dd}, J=8.2,7.2 \mathrm{~Hz}, 2 \mathrm{H}), 7.79-7.68(\mathrm{~m}, 5 \mathrm{H}), 7.46(\mathrm{~d}, J=7.9 \mathrm{~Hz}, 1 \mathrm{H}), 7.40-7.22(\mathrm{~m}$, $8 \mathrm{H}), 5.71(\mathrm{~d}, J=21.0 \mathrm{~Hz}, 2 \mathrm{H}) .{ }^{13} \mathrm{C} \mathrm{NMR}\left(101 \mathrm{MHz}, \mathrm{CDCl}_{3}\right) \delta 159.2,156.8,143.5$, 143.0, 140.7, 140.2, 139.7, 139.2, 137.9, 131.1, 130.0, 129.7, 129.0, 128.9, 128.8, 127.1, 126.2, 124.0, 122.0, 121.4, 121.1, 120.9, 120.2, 116.1, 110.3, 51.0. ESI-HRMS (m/z): calcd. For $\mathrm{C}_{46} \mathrm{H}_{27} \mathrm{~N}_{4} \mathrm{O}_{2}[\mathrm{M}+\mathrm{H}]^{+} 667.2129$, found 667.2131 .

\subsection{Synthesis of $\mathrm{Cz}-\mathrm{NpaBr}$}

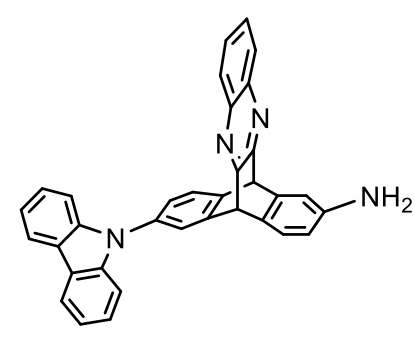

$\mathrm{Cz}-\mathrm{NH}_{2}$

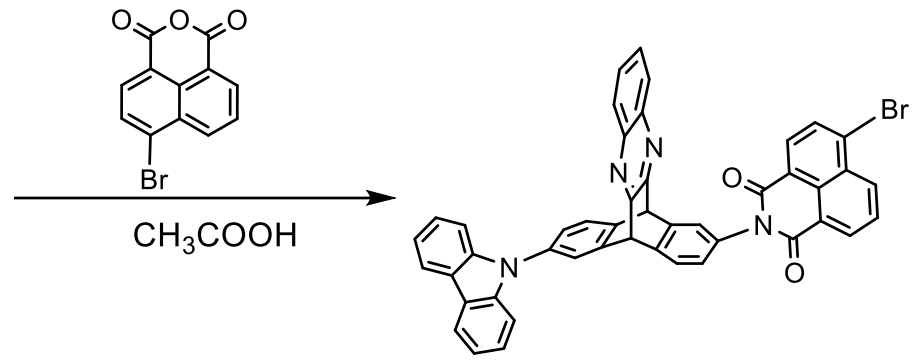

$\mathrm{Cz}-\mathrm{NpaBr}$

A similar procedure for $\mathbf{C z}-\mathbf{N p a}$ was performed to give $\mathbf{C z}-\mathbf{N p a B r}$ as a yellowish solid (50 mg, 35\%). Melting point: $493 \mathrm{~K} .{ }^{1} \mathrm{H}$ NMR (400 MHz, $\left.\mathrm{CDCl}_{3}\right) \delta 8.96(\mathrm{~d}, J=$ 8.0, 2H), $8.72(\mathrm{~d}, J=7.8 \mathrm{~Hz}, 2 \mathrm{H}), 8.45-8.38(\mathrm{~m}, 1 \mathrm{H}), 8.11(\mathrm{~d}, J=7.6 \mathrm{~Hz}, 2 \mathrm{H}), 7.98$ (ddd, $J=10.2,5.0,3.4 \mathrm{~Hz}, 2 \mathrm{H}), 7.79-7.65(\mathrm{~m}, 5 \mathrm{H}), 7.45(\mathrm{~d}, J=7.8 \mathrm{~Hz}, 1 \mathrm{H}), 7.38-$ $7.22(\mathrm{~m}, 8 \mathrm{H}), 5.75-5.64(\mathrm{~d}, J=20.8 \mathrm{~Hz}, 2 \mathrm{H}) .{ }^{13} \mathrm{C} \mathrm{NMR}\left(101 \mathrm{MHz}, \mathrm{CDCl}_{3}\right) \delta 159.7$, 153.3, 144.3, 143.7, 140.8, 140.4, 139.5, 139.4, 138.9, 136.5, 128.3, 127.7, 126.7, 126.6, 126.4, 125.6, 125.4, 125.2, 124.6, 123.8, 123.4, 120.6, 120.3, 120.1, 119.0, 118.9, 121.3, 109.9, 54.9. ESI-HRMS (m/z): calcd. for $\mathrm{C}_{46} \mathrm{H}_{25} \mathrm{BrN}_{4} \mathrm{O}_{2}[\mathrm{M}+\mathrm{H}]^{+}$745.1234, found 745.1228 .

\subsection{Synthesis of Pz-BPI}

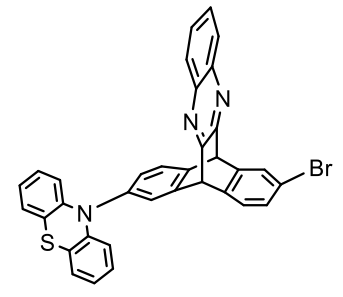

$\mathrm{Pz}-\mathrm{Br}$

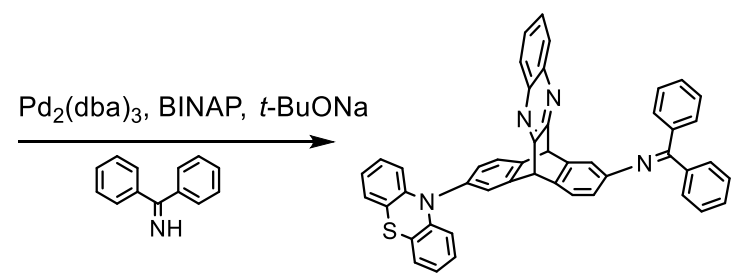

Pz-BPI

A similar procedure for $\mathbf{C z - B P I}$ was performed to give Pz-BPI as a yellowish solid $(60 \mathrm{mg}, 78 \%) .{ }^{1} \mathrm{H} \mathrm{NMR}\left(400 \mathrm{MHz}, d_{6}\right.$-DMSO) $\delta 8.28(\mathrm{~d}, J=7.3 \mathrm{~Hz}, 2 \mathrm{H}), 8.10-8.00$ $(\mathrm{m}, 2 \mathrm{H}), 7.90(\mathrm{dd}, J=4.9,2.9 \mathrm{~Hz}, 2 \mathrm{H}), 7.78-7.71(\mathrm{~m}, 2 \mathrm{H}), 7.69-7.62(\mathrm{~m}, 2 \mathrm{H}), 7.57$ (d, $J=7.1 \mathrm{~Hz}, 1 \mathrm{H}), 7.55-7.38(\mathrm{~m}, 6 \mathrm{H}), 7.34-7.28(\mathrm{~m}, 4 \mathrm{H}), 7.27-7.21(\mathrm{~m}, 4 \mathrm{H}), 7.16(\mathrm{dd}, J$ $=7.1,2.1 \mathrm{~Hz}, 2 \mathrm{H}), 6.76(\mathrm{dd}, J=6.9,2.1 \mathrm{~Hz}, 1 \mathrm{H}), 5.83(\mathrm{~d}, J=16.1 \mathrm{~Hz}, 2 \mathrm{H})$. 


\subsection{Synthesis of $\mathrm{Pz}-\mathrm{NH}_{2}$}

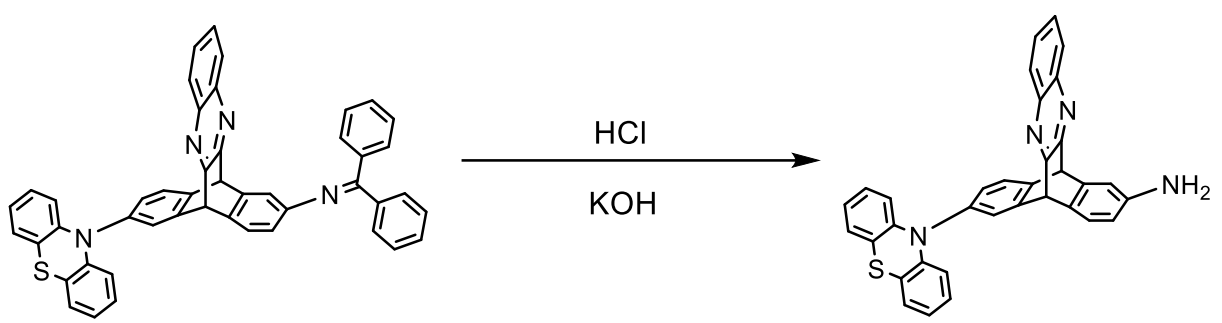

Pz-BPI

$\mathrm{Pz}-\mathrm{NH}_{2}$

A similar procedure for $\mathbf{C z}-\mathbf{N H}_{\mathbf{2}}$ was performed to give $\mathbf{P z}-\mathbf{N H}_{\mathbf{2}}$ as an yellowish solid ( $35 \mathrm{mg}, 79 \%) .{ }^{1} \mathrm{H}$ NMR (400 MHz, $\mathrm{CDCl}_{3}$ ) $\delta$ 8.01-7.96 (m, 2H), 7.81 (d, $J=1.9$ $\mathrm{Hz}, 1 \mathrm{H}), 7.72-7.68(\mathrm{~m}, 2 \mathrm{H}), 7.59(\mathrm{~d}, J=2.1 \mathrm{~Hz}, 1 \mathrm{H}), 7.47$ (d, $J=7.2 \mathrm{~Hz}, 2 \mathrm{H}), 7.31$ $(\mathrm{dd}, J=7.9,2.0 \mathrm{~Hz}, 2 \mathrm{H}), 7.18(\mathrm{dd}, J=7.7,2.2 \mathrm{~Hz}, 1 \mathrm{H}), 7.11-7.02(\mathrm{~m}, 2 \mathrm{H}), 6.90(\mathrm{~d}, J$ $=1.3 \mathrm{~Hz}, 2 \mathrm{H}), 6.29-6.20(\mathrm{~m}, 2 \mathrm{H}), 5.79(\mathrm{~s}, 1 \mathrm{H}), 5.64(\mathrm{~d}, J=16.2 \mathrm{~Hz}, 2 \mathrm{H}), 3.38(\mathrm{~s}, 2 \mathrm{H})$. ${ }^{13} \mathrm{C}$ NMR (101 MHz, $d_{6}$-DMSO) $\delta 173.3,165.4,157.1,145.1,140.5,136.6,134.6$, $130.4,126.7,123.2,120.8,110.2,53.4$.

\subsection{Synthesis of Pz-Npa}

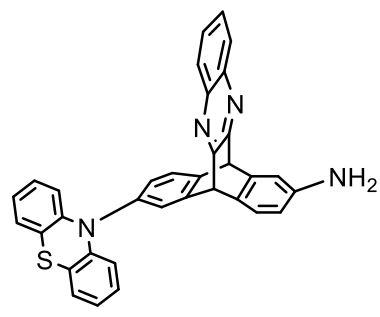

$\mathrm{Pz}-\mathrm{NH}_{2}$

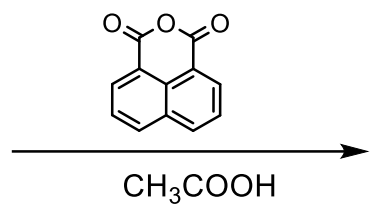

Pz-Npa

A similar procedure for $\mathbf{C z}-\mathbf{N p a}$ was performed to give $\mathbf{P z}-\mathbf{N p a}$ as a yellowish solid (39 mg, 29\%). Melting point: $473 \mathrm{~K} .{ }^{1} \mathrm{H} \mathrm{NMR}\left(400 \mathrm{MHz}, \mathrm{CDCl}_{3}\right) \delta 8.56(\mathrm{~d}, J=7.6 \mathrm{~Hz}$, $2 \mathrm{H}), 8.27-8.23(\mathrm{~d}, J=8.0 \mathrm{~Hz}, 2 \mathrm{H}), 7.88(\mathrm{~m}, 2 \mathrm{H}), 7.79-7.72(\mathrm{~m}, 3 \mathrm{H}), 7.68-7.59(\mathrm{~m}$, 4H), $7.47(\mathrm{~s}, 1 \mathrm{H}), 7.35(\mathrm{~d}, J=8.0 \mathrm{~Hz}, 1 \mathrm{H}), 7.26-7.22(\mathrm{dd}, J=7.6,1.6 \mathrm{~Hz}, 1 \mathrm{H}), 7.10$ (dd, $J=7.8,2.0 \mathrm{~Hz}, 1 \mathrm{H}), 6.98-6.89(\mathrm{~m}, 2 \mathrm{H}), 6.75-6.69(\mathrm{~m}, 3 \mathrm{H}), 6.11-6.07(\mathrm{~m}, 1 \mathrm{H})$, $5.65-5.51(\mathrm{~d}, J=19.8 \mathrm{~Hz}, 2 \mathrm{H}) .{ }^{13} \mathrm{C}$ NMR $\left(101 \mathrm{MHz}, d_{6}\right.$-DMSO) $\delta 159.3,156.7,144.4$, 143.7, 143.0, 140.2, 139.5, 137.8, 137.5, 136.9, 133.2, 130.3, 128.9, 128.4, 127.4, 126.1, 125.8, 124.0, 122.1, 122.0, 117.8, 52.9. ESI-HRMS (m/z): calcd. for $\mathrm{C}_{46} \mathrm{H}_{26} \mathrm{~N}_{4} \mathrm{O}_{2} \mathrm{~S}$ $[\mathrm{M}+\mathrm{H}]^{+}$699.1849, found 699.1842. 


\subsection{Synthesis of Pz-NpaBr}

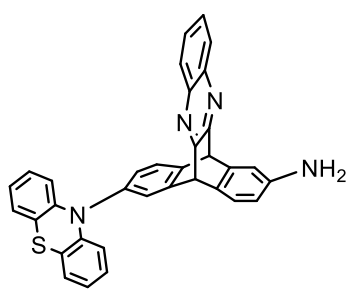

Pz-NH2

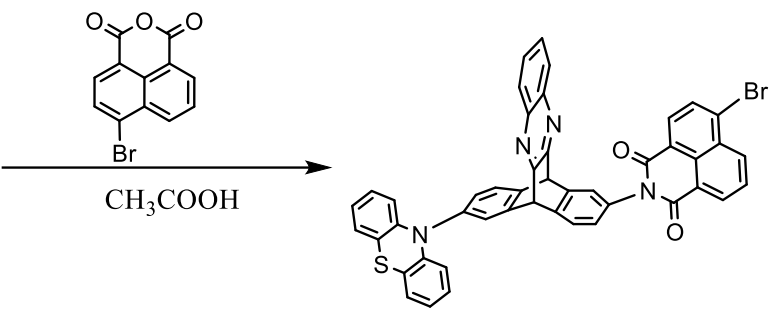

Pz-NpaBr

A similar procedure for $\mathbf{C z}-\mathbf{N p a}$ was performed to give $\mathbf{P z}-\mathbf{N p a B r}$ as a yellowish solid (55 mg, 37\%). Melting point: $485 \mathrm{~K} .{ }^{1} \mathrm{H} \mathrm{NMR}\left(400 \mathrm{MHz}, \mathrm{CDCl}_{3}\right) \delta 8.88(\mathrm{~m}, 2 \mathrm{H})$, $8.43(\mathrm{~d}, J=7.8 \mathrm{~Hz}, 1 \mathrm{H}), 8.05(\mathrm{~d}, J=7.6 \mathrm{~Hz}, 1 \mathrm{H}), 7.89(\mathrm{~m}, 3 \mathrm{H}), 7.84-7.76(\mathrm{~m}, 1 \mathrm{H})$, $7.72-7.61(\mathrm{~m}, 3 \mathrm{H}), 7.54-7.45(\mathrm{~m}, 1 \mathrm{H}), 7.39(\mathrm{~d}, J=8.0 \mathrm{~Hz}, 1 \mathrm{H}), 7.22-7.10(\mathrm{~m}, 1 \mathrm{H})$, $7.03(\mathrm{dd}, J=7.8,2.0 \mathrm{~Hz}, 1 \mathrm{H}), 6.90-6.83(\mathrm{~m}, 2 \mathrm{H}), 6.73-6.64(\mathrm{~m}, 4 \mathrm{H}), 6.11-6.07(\mathrm{~m}$, 2H), $5.55(\mathrm{~d}, J=21.0 \mathrm{~Hz}, 2 \mathrm{H}) .{ }^{13} \mathrm{C}$ NMR $\left(101 \mathrm{MHz}, \mathrm{CDCl}_{3}\right) \delta 160.4,157.6,145.4$, $143.8,143.1,140.3,139.0,137.0,136.9,136.8,135.4,133.4,131.7,131.6,130.8,129.9$, $128.9,128.8,128.2$, 127.3, 126.1, 125.8, 124.4, 122.6, 121.9, 120.9, 117.9, 57.4. ESIHRMS (m/z): calcd. for $\mathrm{C}_{46} \mathrm{H}_{25} \mathrm{BrN}_{4} \mathrm{O}_{2} \mathrm{~S}[\mathrm{M}+\mathrm{H}]^{+} 777.0954$, found 777.0949.

\section{References:}

[1] Yan-Zuo Lin, Chiung Hui Huang, Yuan Jay Chang, Chia-Wei Yeh, Tsung-Mei Chin, Kai-Ming Chi, Po-Ting Chou, Motonori Watanabe, Tahsin J. Chow. Anthracene based organic dipolar compounds for sensitized solar cells. Tetrahedron, 2014, 70(2): 262-269.

[2] Đani Škalamera, Jelena Veljković, Lucija Ptiček, Matija Sambol, Kata Mlinarić-Majerski, Nikola Basarić. Synthesis of asymmetrically disubstituted anthracenes. Tetrahedron, 2017, 73(40): 5892-5899.

[3] Cally Li, Soren K. Mellerup, Xiang Wang and Suning Wang. Accessing Two-Stage Regioselective Photoisomerization in Unsymmetrical N,C-Chelate Organoboron Compounds: Reactivity of B(ppz)(Mes)Ar. Organometallics, 2018, 37, 3360-3367.

[4] Wen-Li Jia, Dong-Ren Bai, T McCormick, Qin-De Liu, Michael Motala, Rui-Yao Wang, Corey Seward, Ye Tao, Suning Wang. Three-coordinate organoboron compounds $\mathrm{BAr}_{2} \mathrm{R}(\mathrm{Ar}=$ mesityl, $\mathrm{R}=7$ azaindolyl- or 2,2'-dipyridylamino-functionalized aryl or thienyl) for electroluminescent devices and supramolecular assembly. Chemistry - A European Journal, 2004, 10, 994-1006.

[5] Xiaofei Xu, Shanghui Ye, Bairong He, Bin Chen, Jiayun Xiang, Jian Zhou, Ping Lu, Zujin Zhao, Huayu Qiu. Dimesitylboryl-functionalized fluorene derivatives: Promising luminophors with good electron-transporting ability for deep blue organic light-emitting diodes. Dyes and Pigments, 2014, 101, 136-141. 


\section{Characterization by NMR and HRMS Spectroscopy}

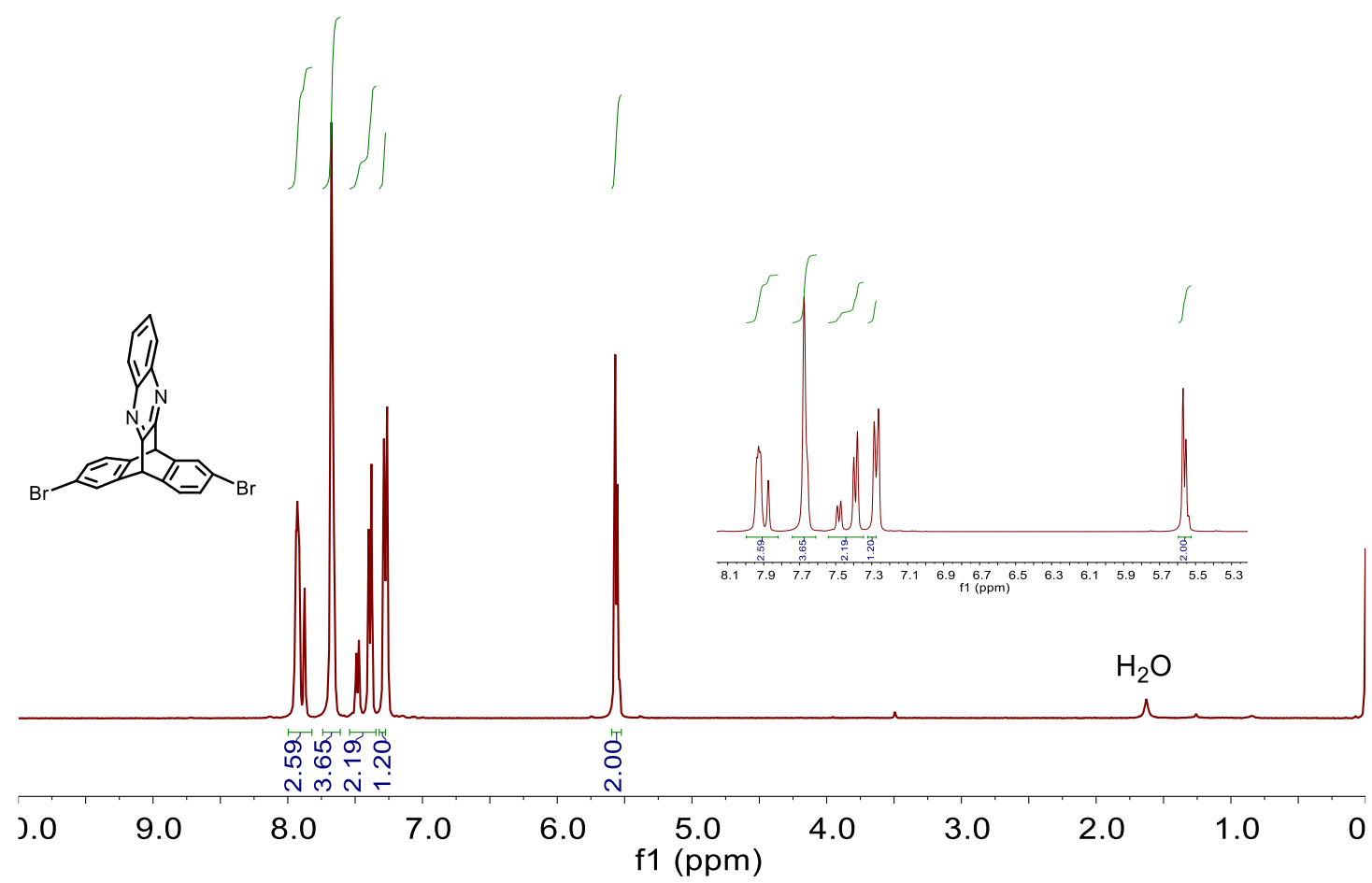

Figure S1. ${ }^{1} \mathrm{H}$ NMR (400 MHz, MeOH-d $)$ spectrum of 6.

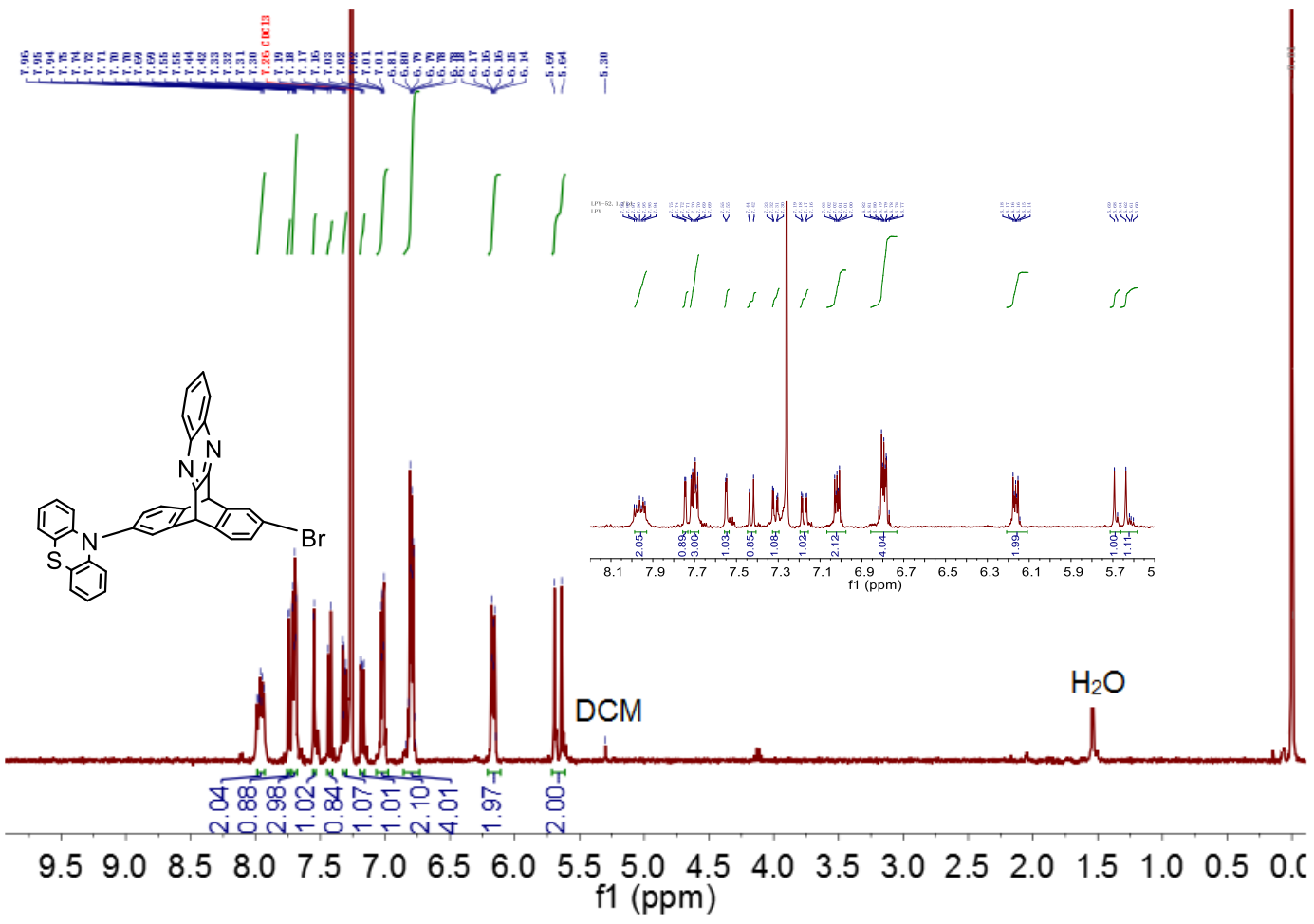

Figure S2. ${ }^{1} \mathrm{H}$ NMR (400 MHz, $\left.\mathrm{CDCl}_{3}\right)$ spectrum of Pz-Br. 


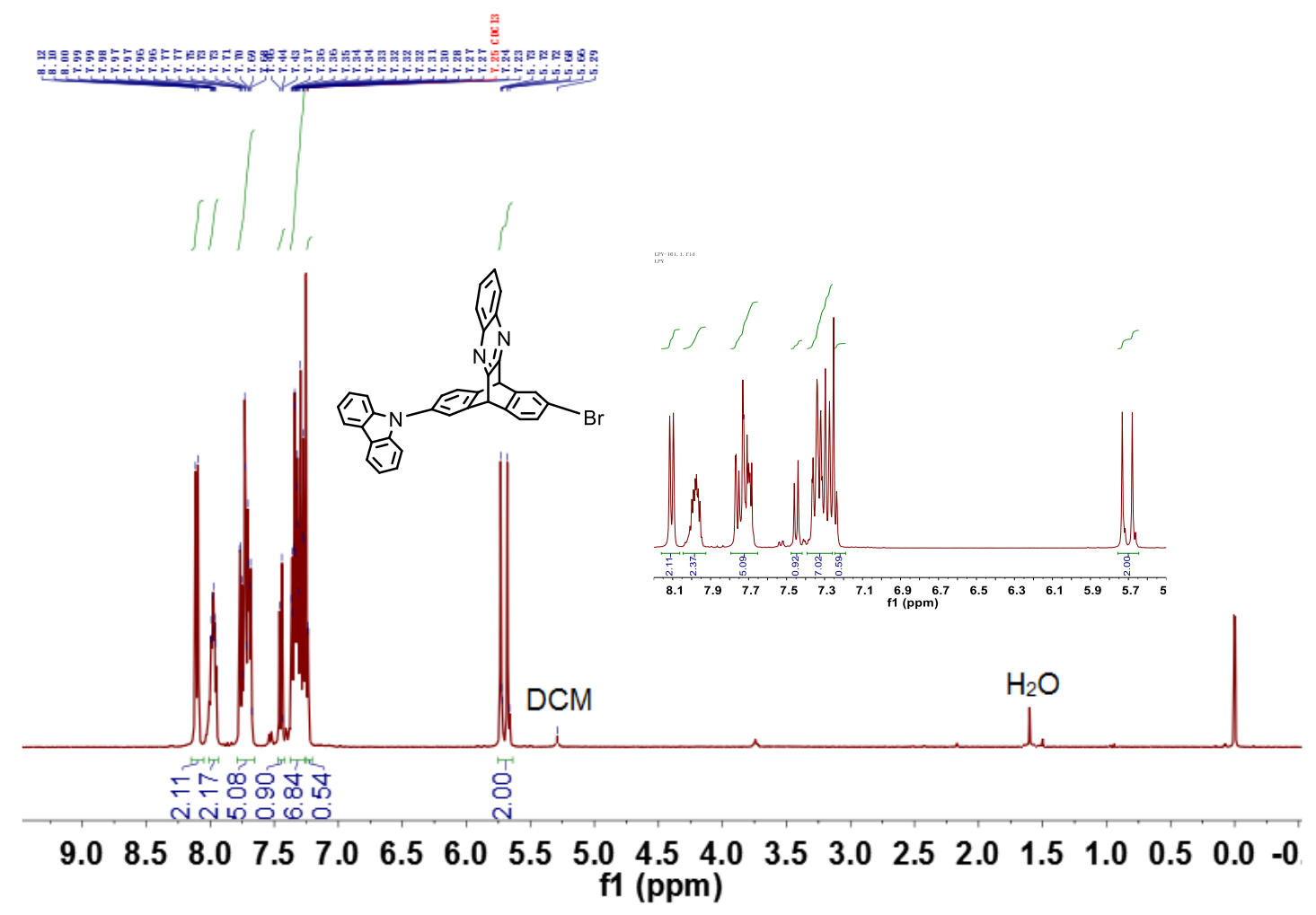

Figure S3. ${ }^{1} \mathrm{H}$ NMR (400 MHz, $\left.\mathrm{CDCl}_{3}\right)$ spectrum of $\mathbf{C z - B r}$.

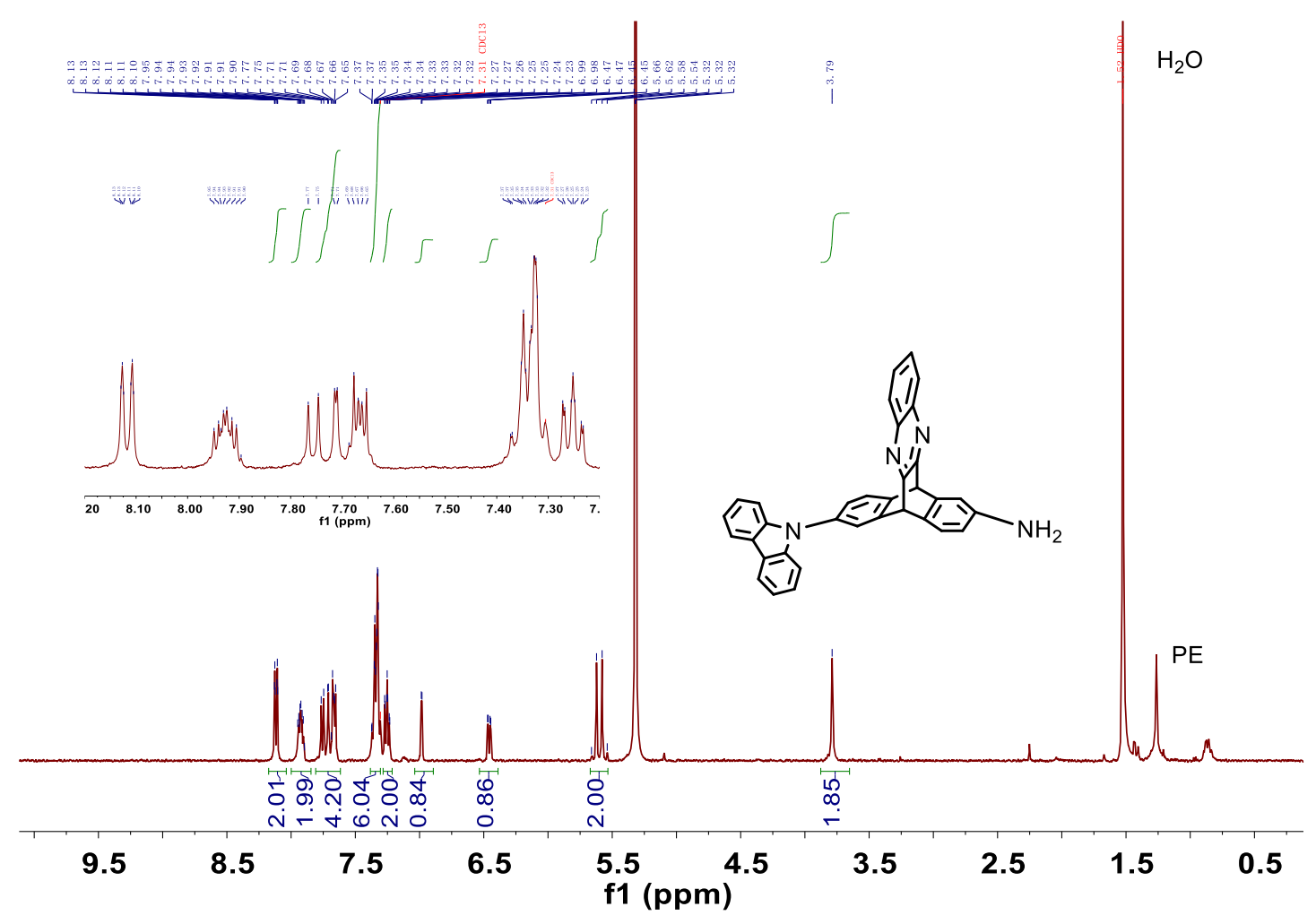

Figure S4. ${ }^{1} \mathrm{H}$ NMR $\left(400 \mathrm{MHz}, \mathrm{CDCl}_{3}\right)$ spectrum of $\mathbf{C z}-\mathbf{N H}_{2}$. 


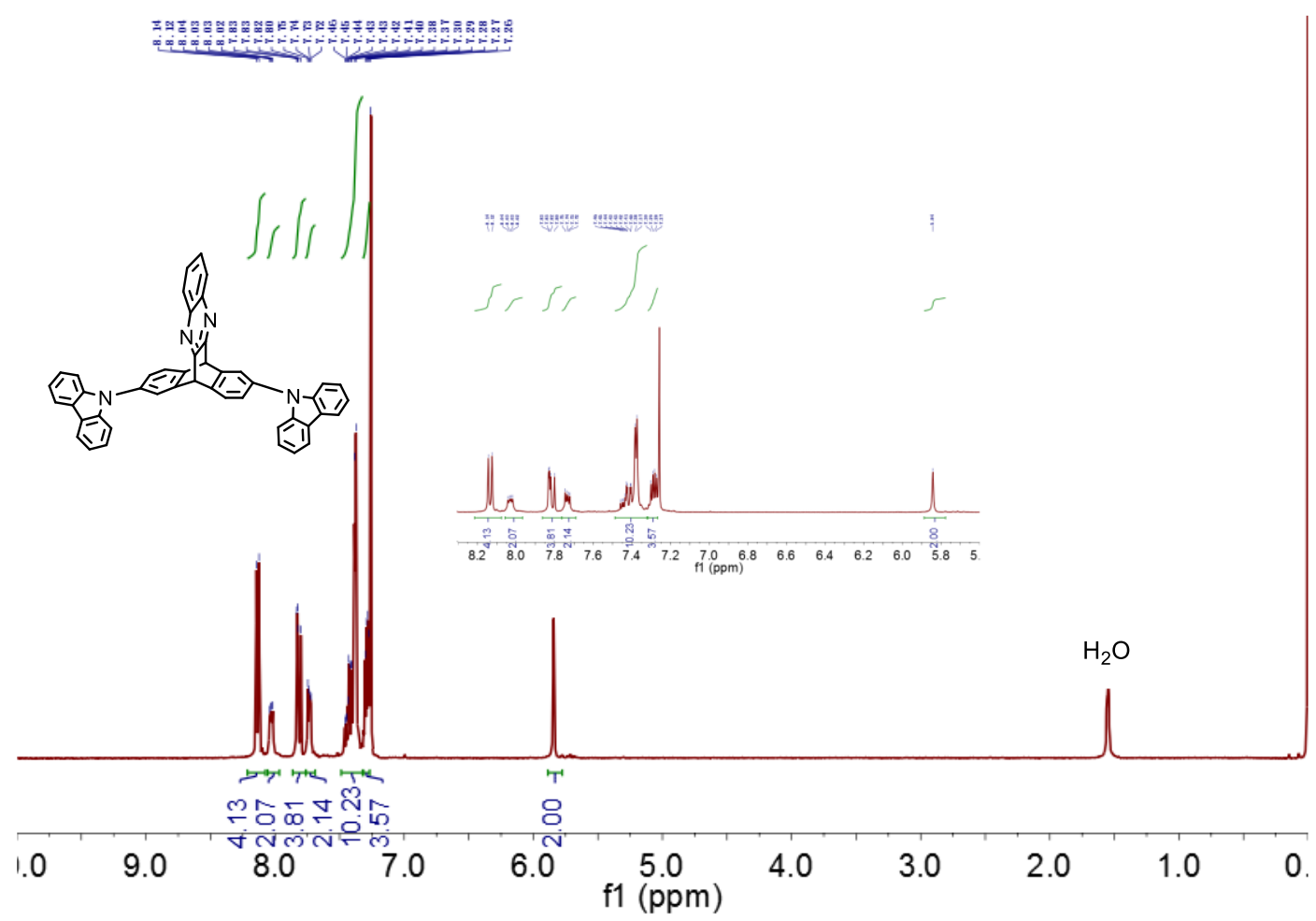

Figure S5. ${ }^{1} \mathrm{H}$ NMR $\left(400 \mathrm{MHz}, \mathrm{CDCl}_{3}\right)$ spectrum of $\mathbf{C z}-\mathbf{C z}$.

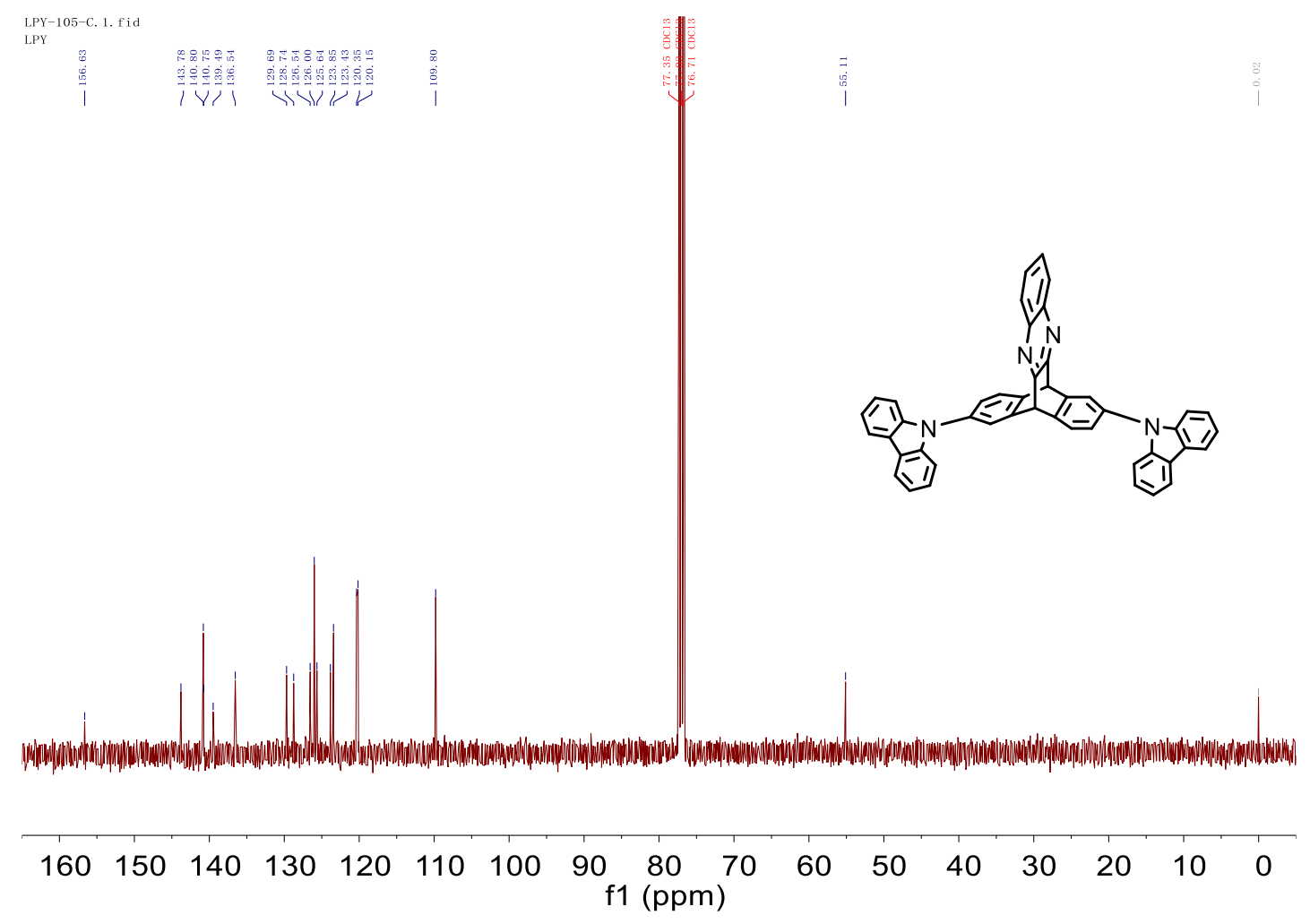

Figure S6. ${ }^{13} \mathrm{C}$ NMR $\left(176 \mathrm{MHz}, \mathrm{CDCl}_{3}\right)$ spectrum of $\mathbf{C z}-\mathbf{C z}$. 


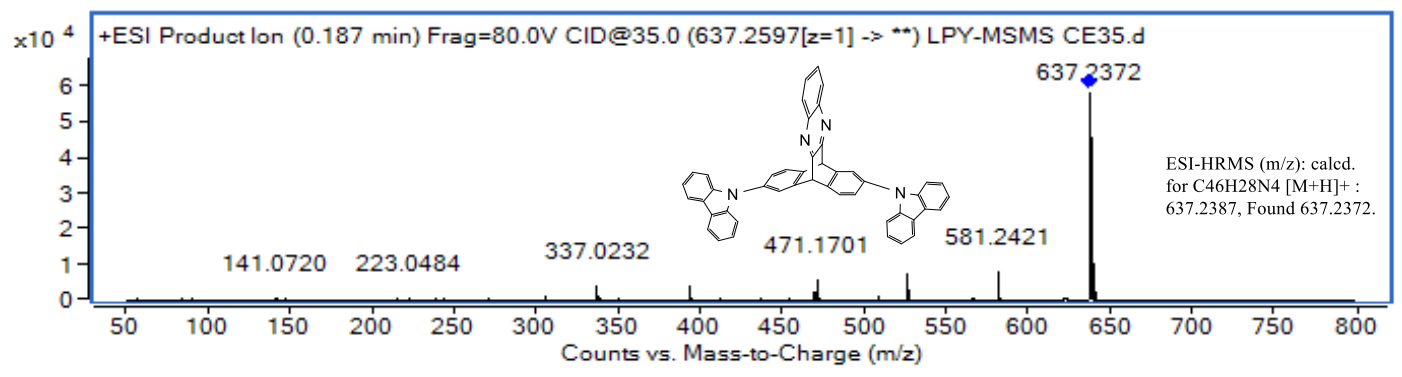

Figure S7. ESI-HRMS spectrum for $\mathbf{C z}-\mathbf{C z}$.

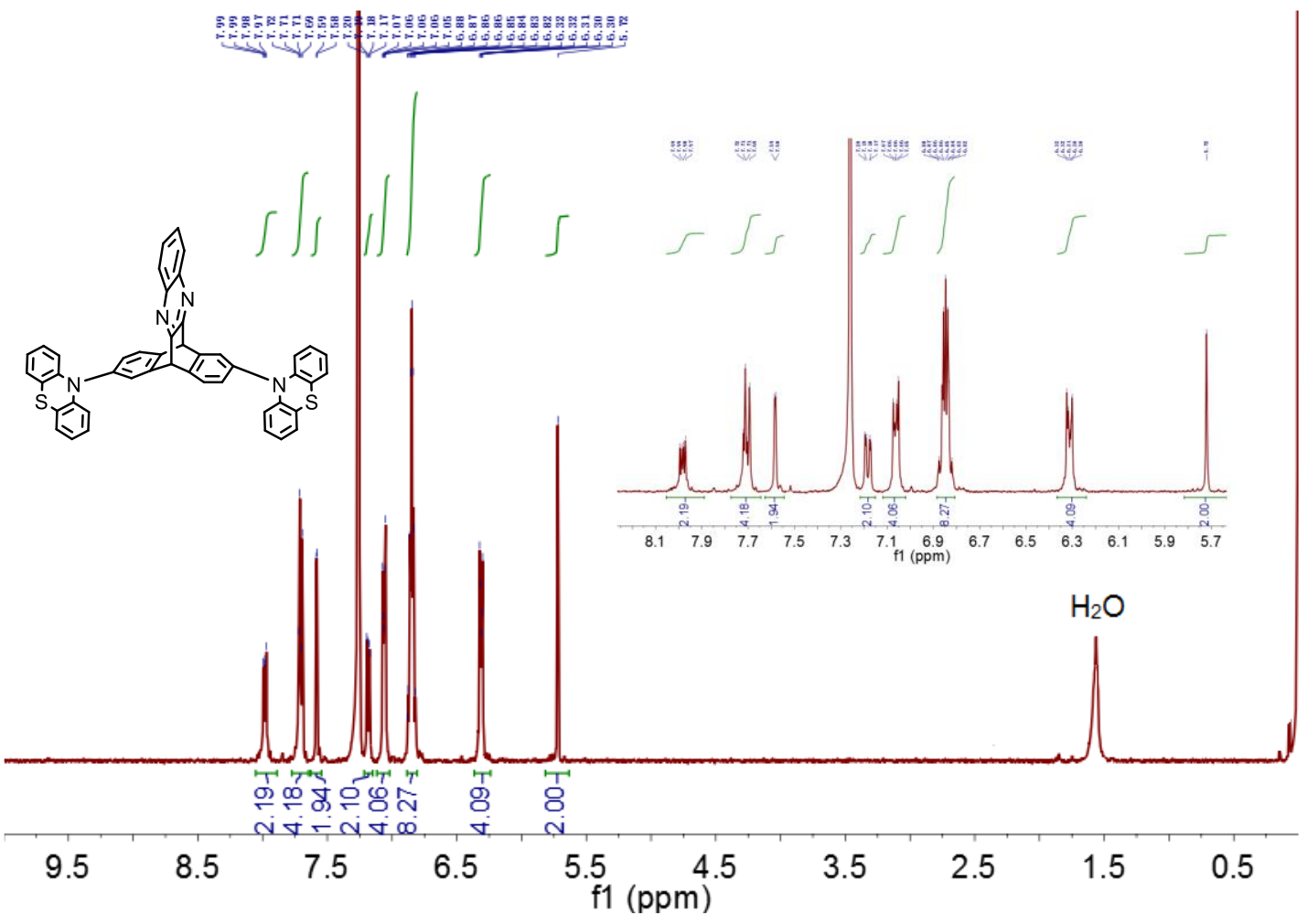

Figure S8. ${ }^{1} \mathrm{H}$ NMR $\left(400 \mathrm{MHz}, \mathrm{CDCl}_{3}\right.$ ) spectrum of Pz-Pz. 


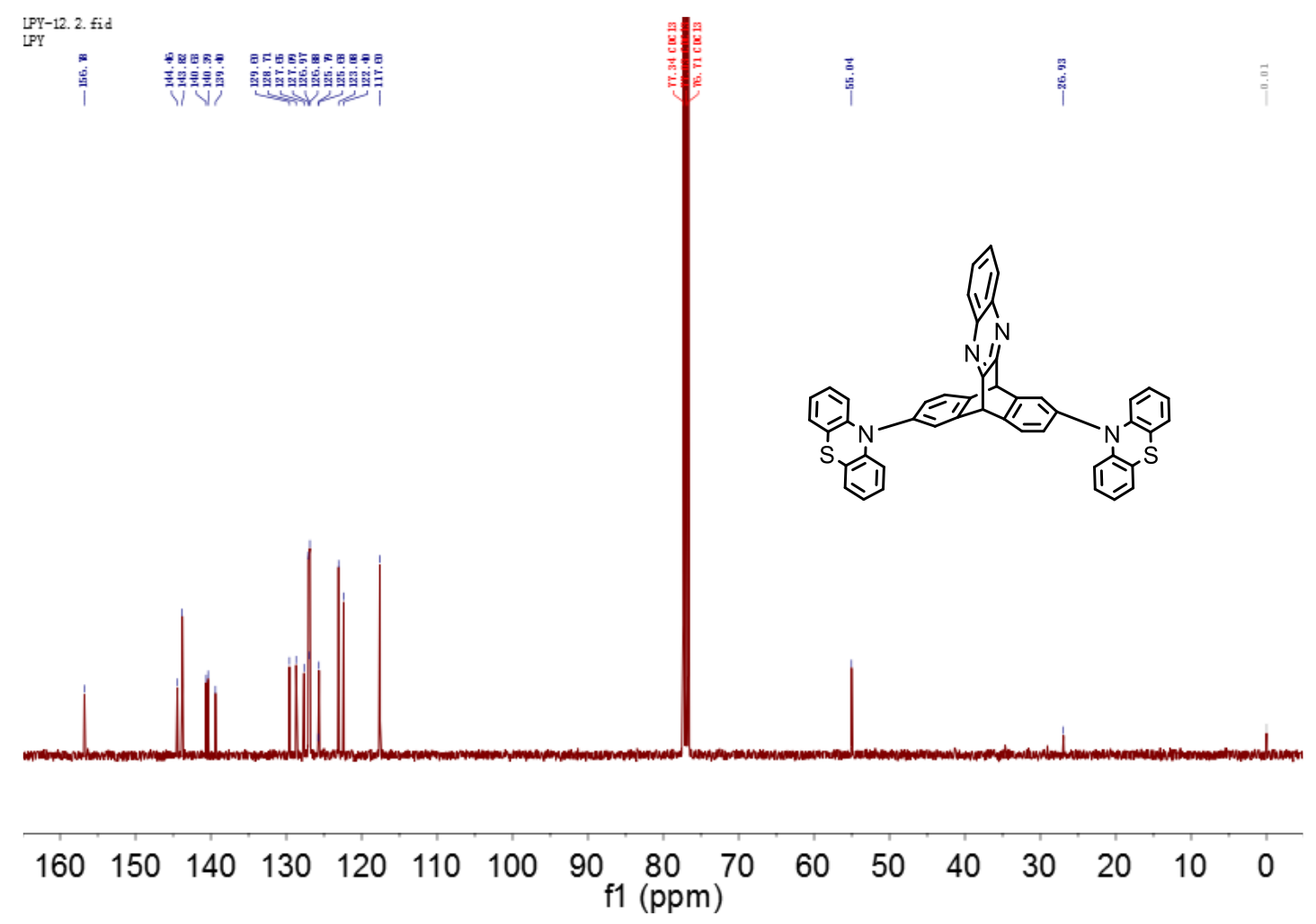

Figure S9. ${ }^{13} \mathrm{C}$ NMR (101 MHz, $\left.\mathrm{CDCl}_{3}\right)$ spectrum of Pz-Pz.

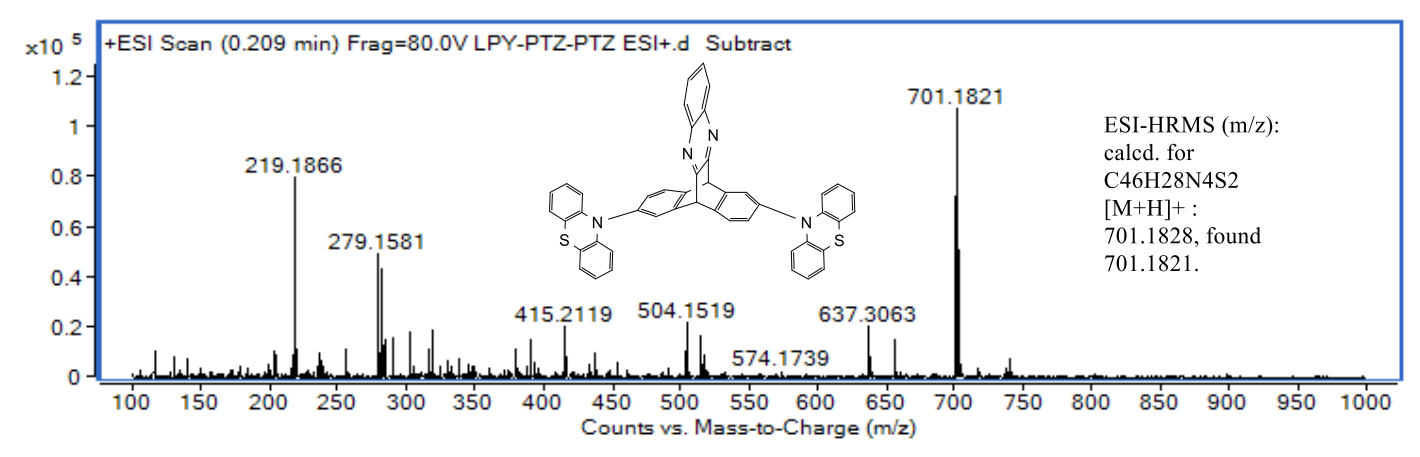

Figure S10. ESI-HRMS spectrum for Pz-Pz. 


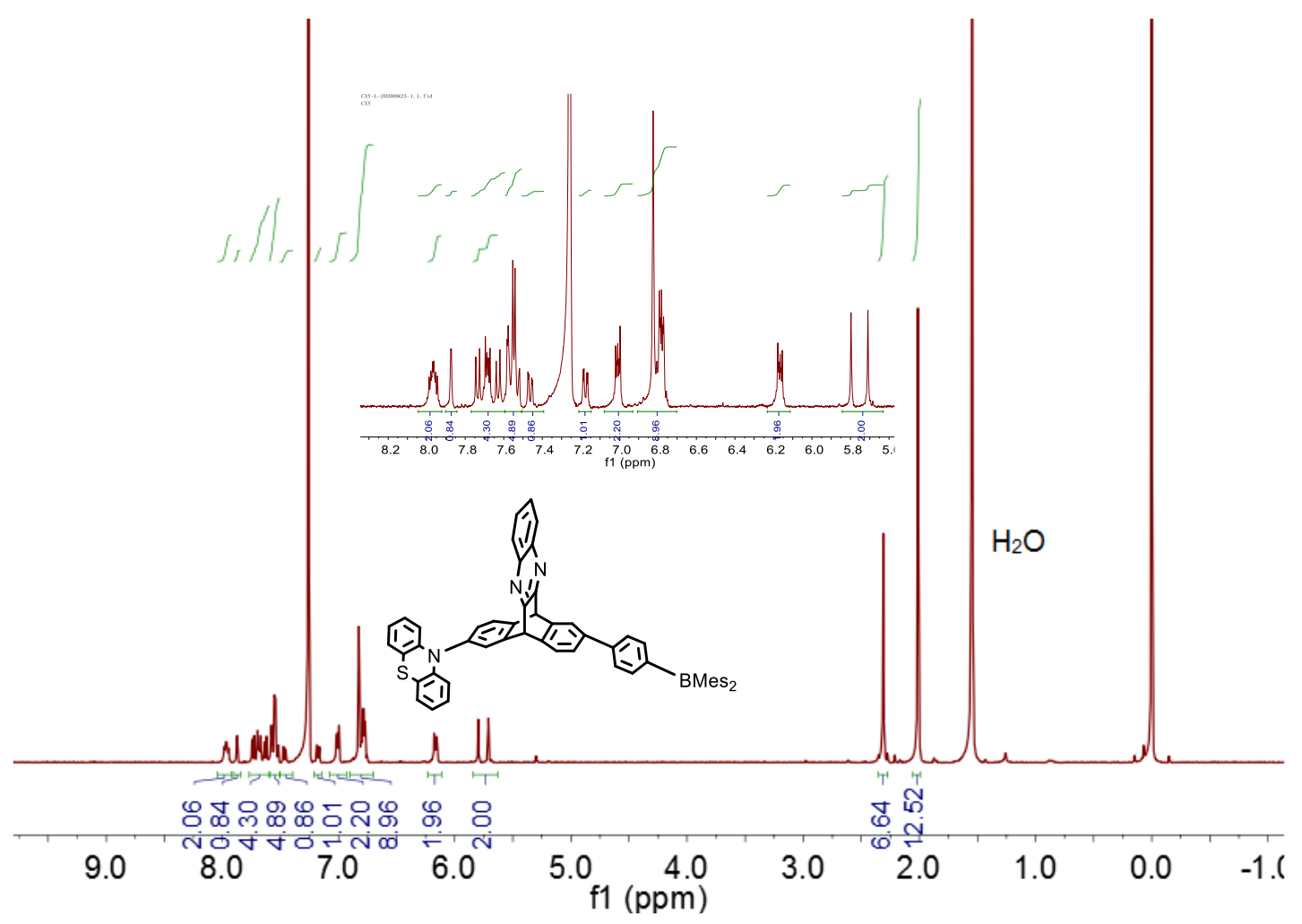

Figure S11. ${ }^{1} \mathrm{H}$ NMR (400 MHz, $\left.\mathrm{CDCl}_{3}\right)$ spectrum of Pz-BMes.
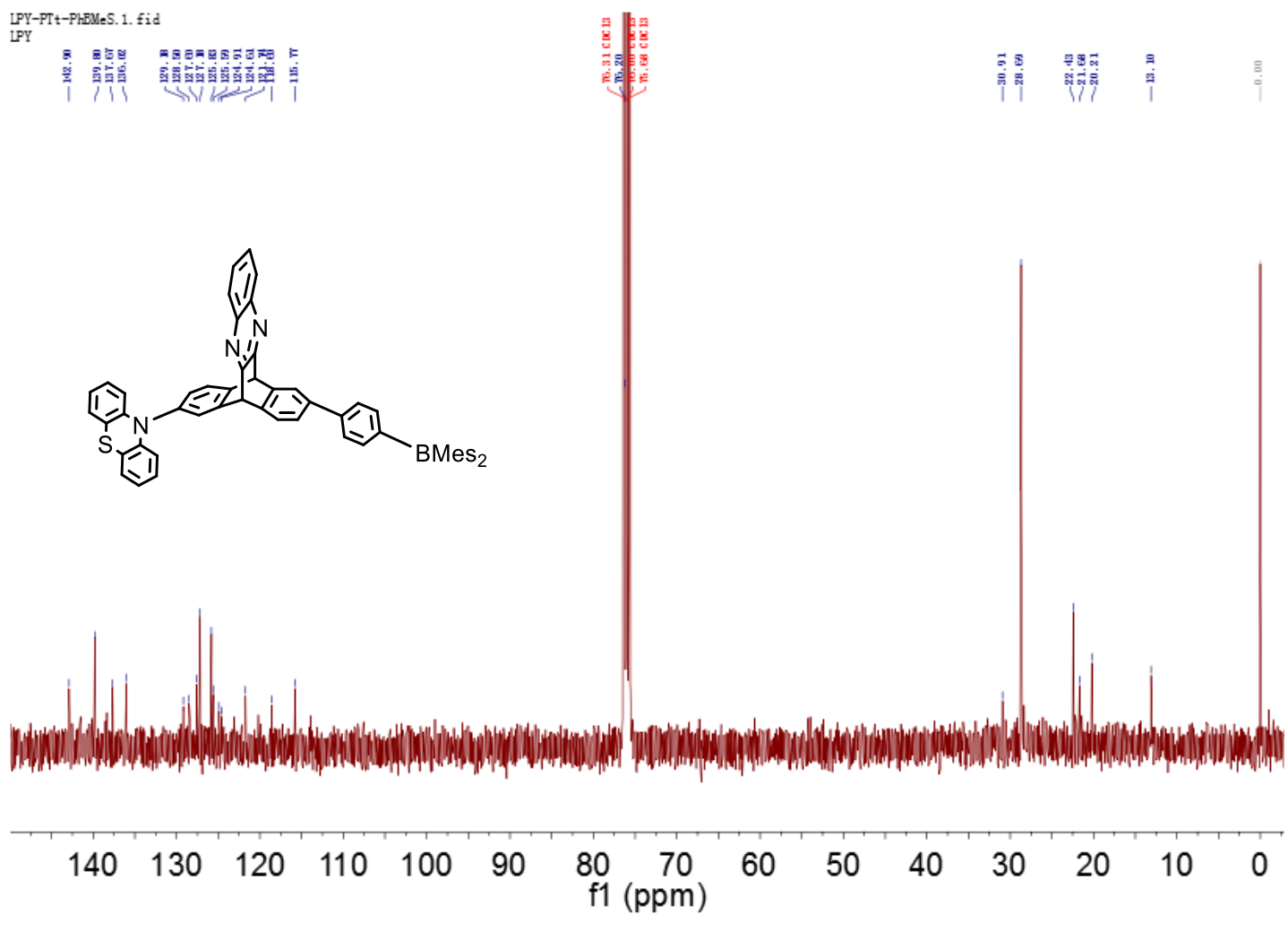

Figure S12. ${ }^{13} \mathrm{C}$ NMR (101 MHz, $\left.\mathrm{CDCl}_{3}\right)$ spectrum of Pz-BMes. 


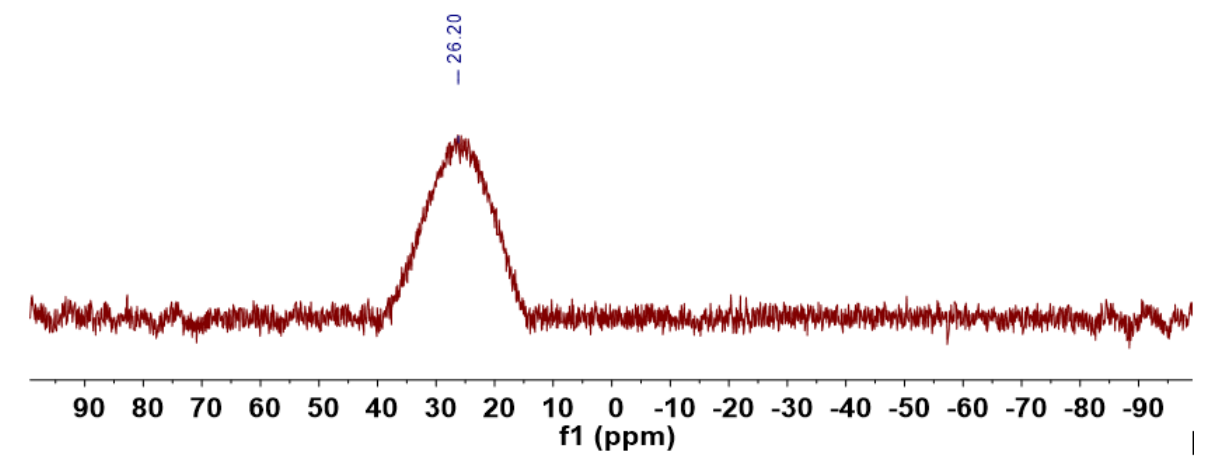

Figure S13. ${ }^{11}$ B NMR (225 MHz, $\left.\mathrm{CDCl}_{3}\right)$ spectrum of Pz-BMes.

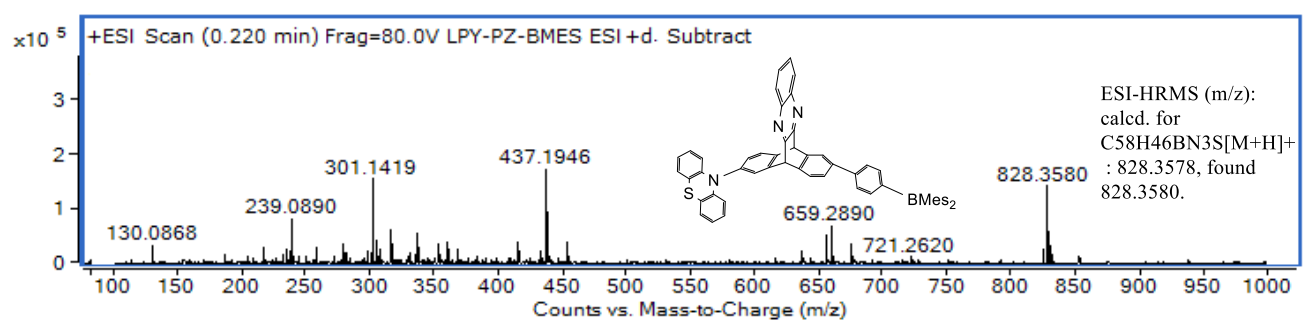

Figure S14. ESI-HRMS spectrum for Pz-BMes.

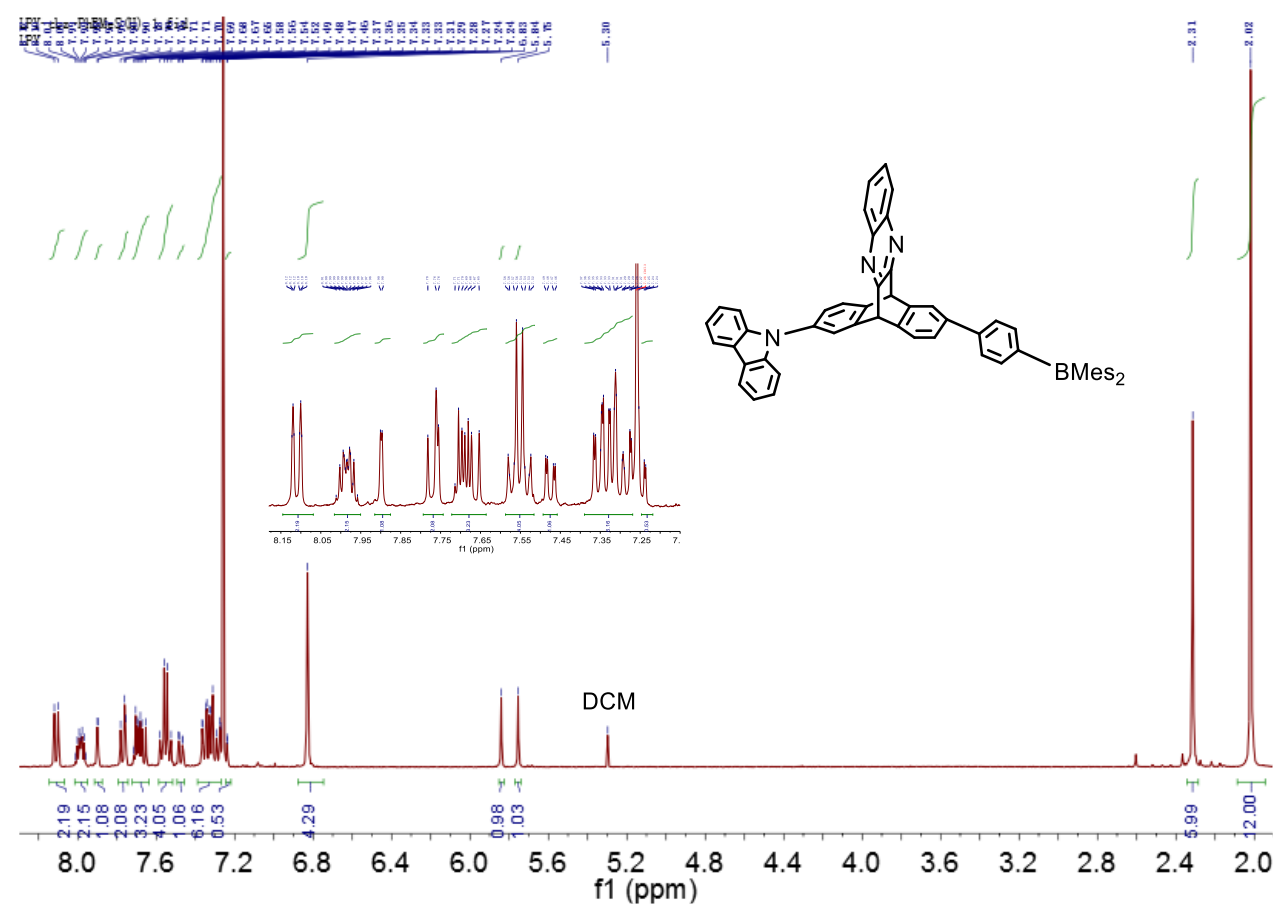

Figure S15. ${ }^{1} \mathrm{H}$ NMR (400 MHz, $\left.\mathrm{CDCl}_{3}\right)$ spectrum of $\mathbf{C z - B M e s}$. 


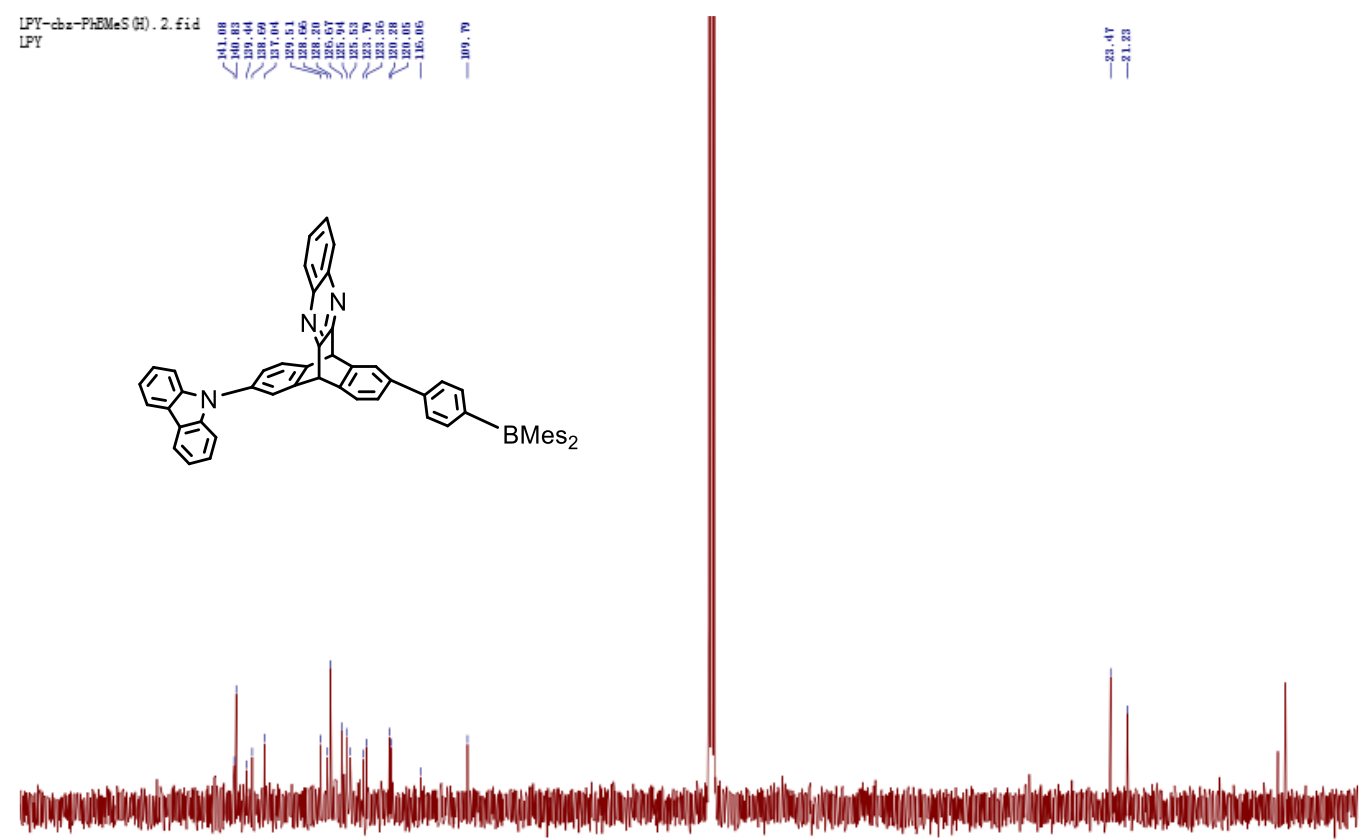

\begin{tabular}{lllllllllllllllllllll}
\hline 0 & 160 & 150 & 140 & 130 & 120 & 110 & 100 & 90 & $\underset{\mathrm{f} 1}{(\mathrm{ppm})} \mathbf{8 0}$ & 70 & 60 & 50 & 40 & 30 & 20 & 10 & 0 & -1
\end{tabular}

Figure S16. ${ }^{13} \mathrm{C}$ NMR (101 MHz, $\left.\mathrm{CDCl}_{3}\right)$ spectrum of Cz-BMes.

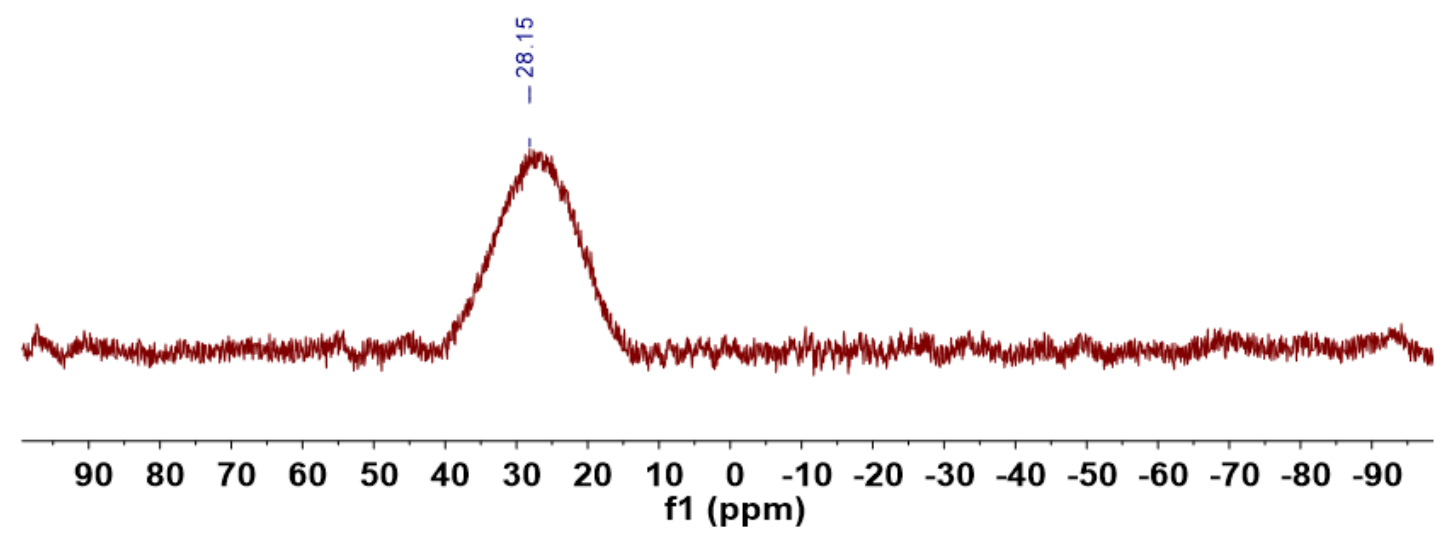

Figure S17. ${ }^{11} \mathrm{~B}$ NMR $\left(225 \mathrm{MHz}, \mathrm{CDCl}_{3}\right)$ spectrum of Cz-BMes.

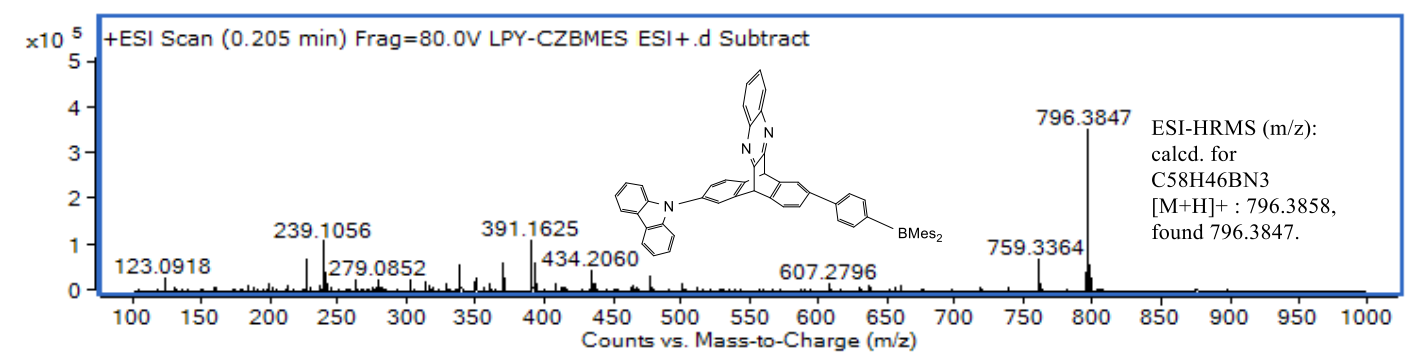

Figure S18. ESI-HRMS spectrum for Cz-BMes. 


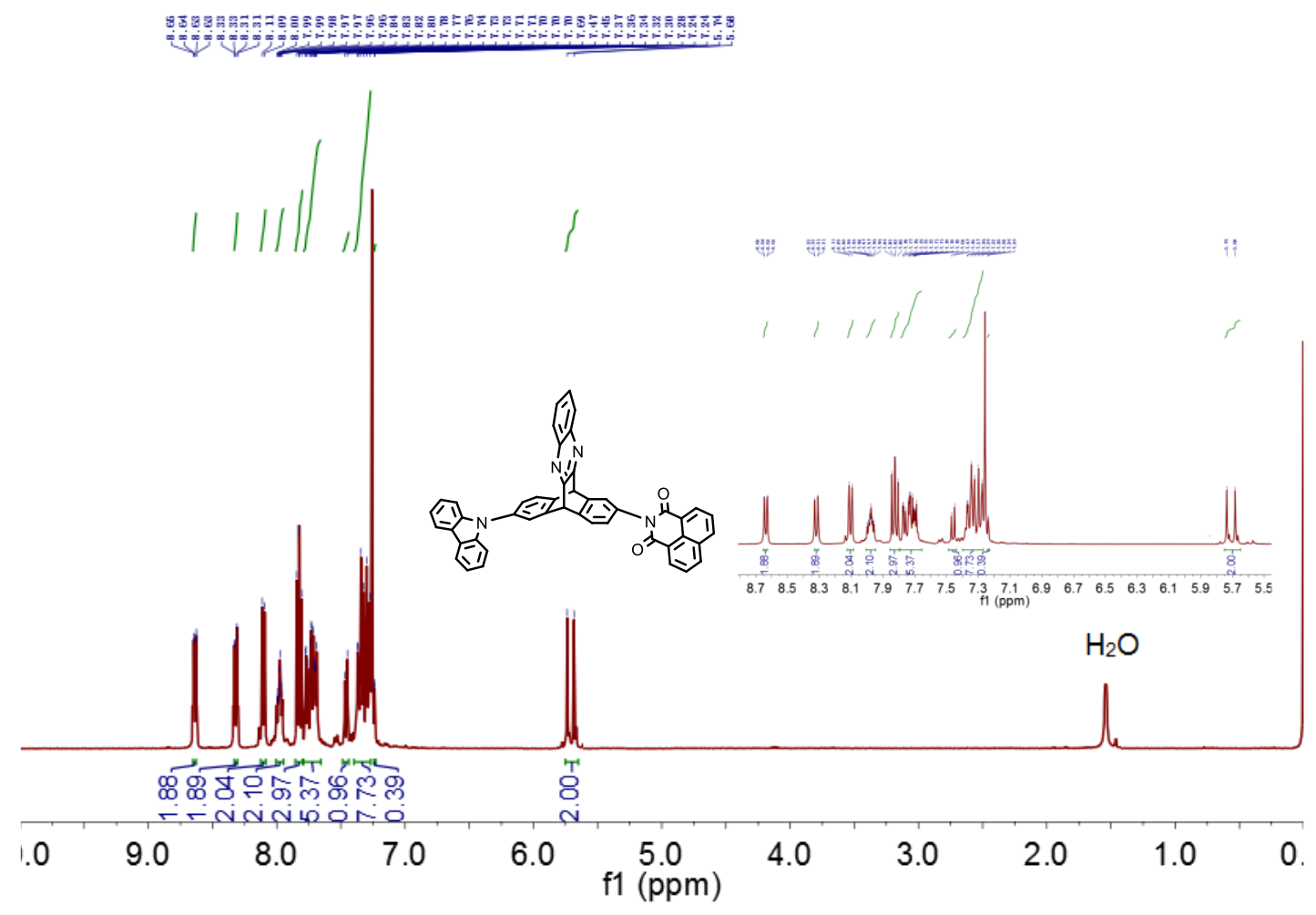

Figure S19. ${ }^{1} \mathrm{H}$ NMR (400 MHz, $\left.\mathrm{CDCl}_{3}\right)$ spectrum of $\mathbf{C z}-\mathbf{N p a}$.

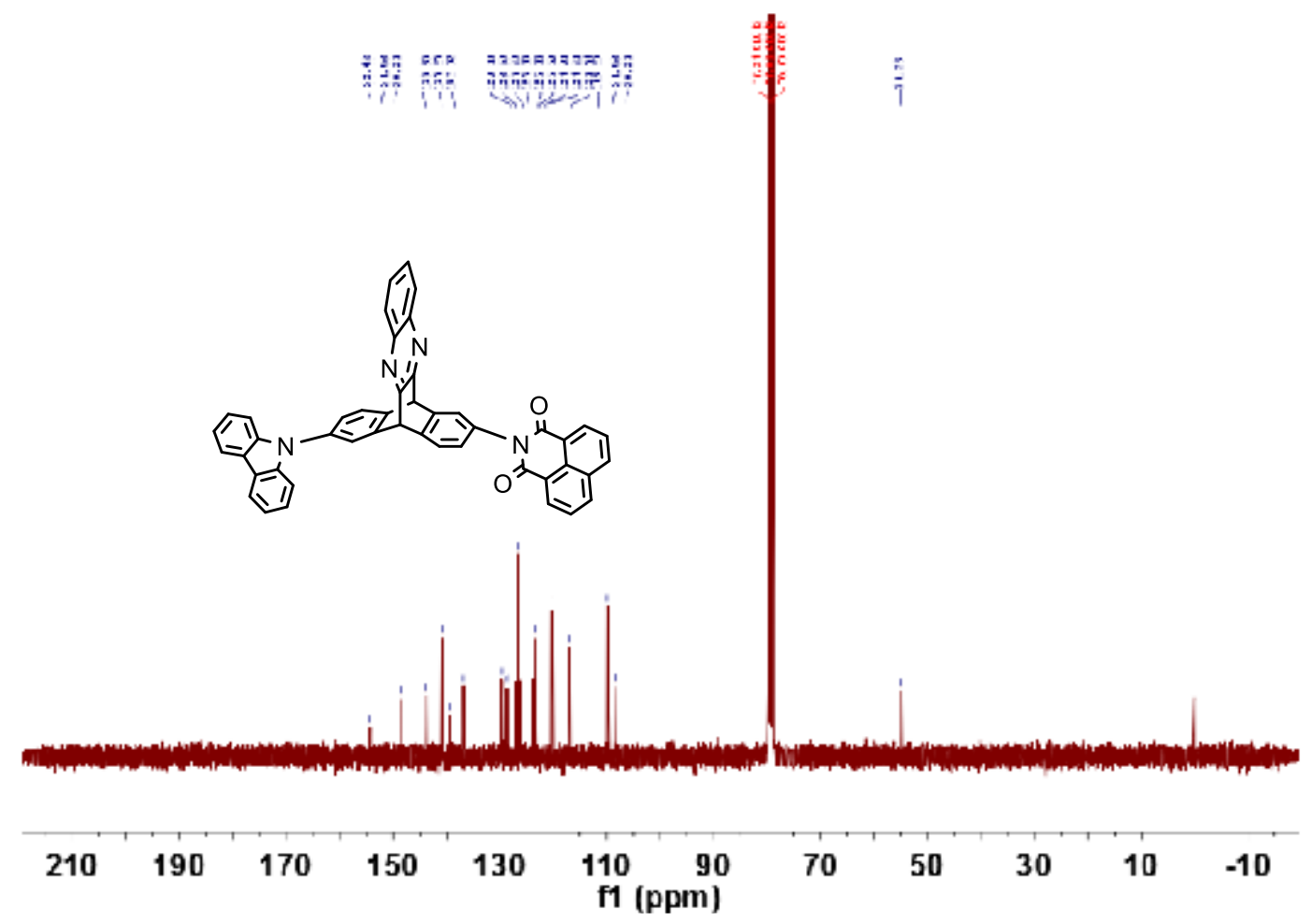

Figure S20. ${ }^{13} \mathrm{C}$ NMR $\left(101 \mathrm{MHz}, \mathrm{CDCl}_{3}\right)$ spectrum of $\mathbf{C z}-\mathbf{N p a}$. 


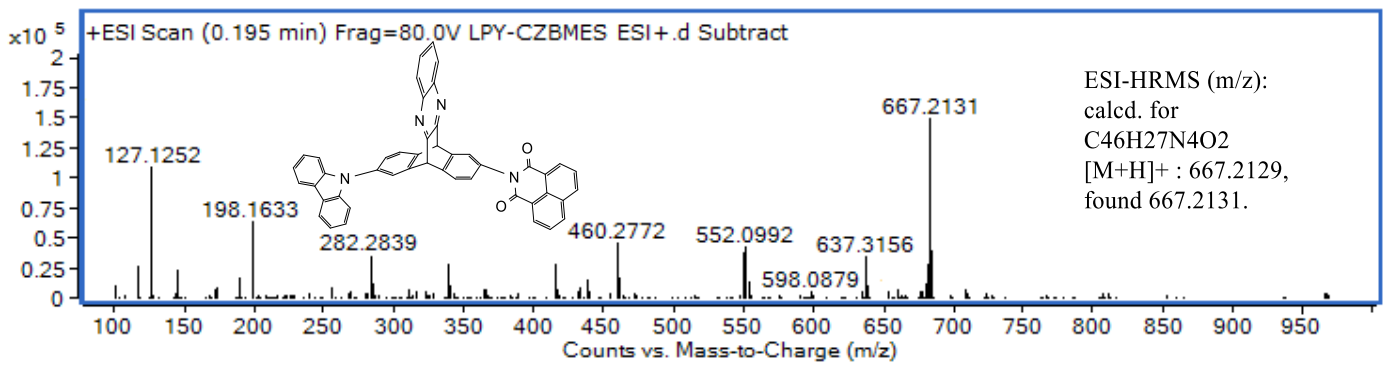

Figure S21. ESI-HRMS spectrum for Cz-Npa.

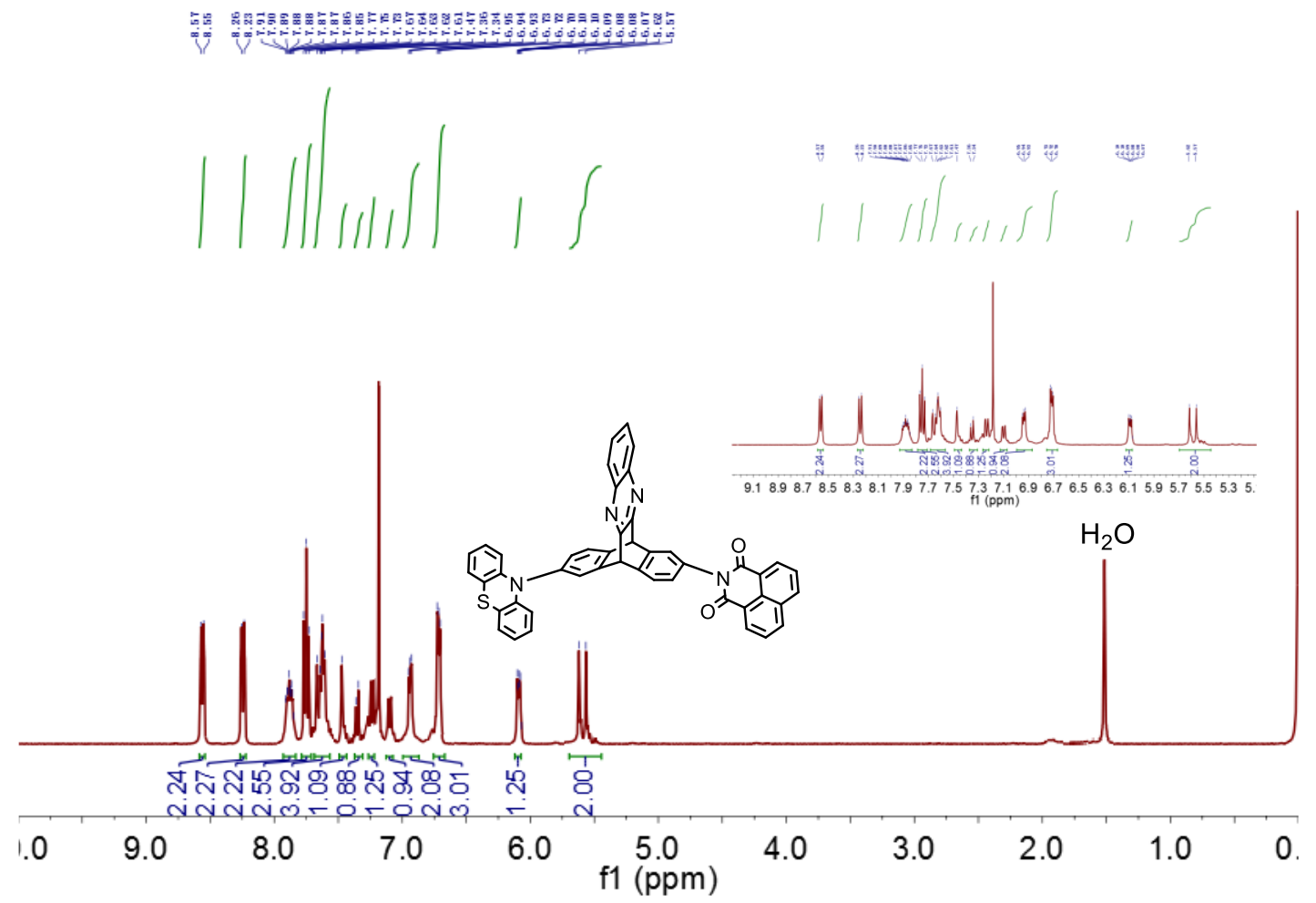

Figure S22. ${ }^{1} \mathrm{H}$ NMR $\left(400 \mathrm{MHz}, \mathrm{CDCl}_{3}\right)$ spectrum of Pz-Npa. 


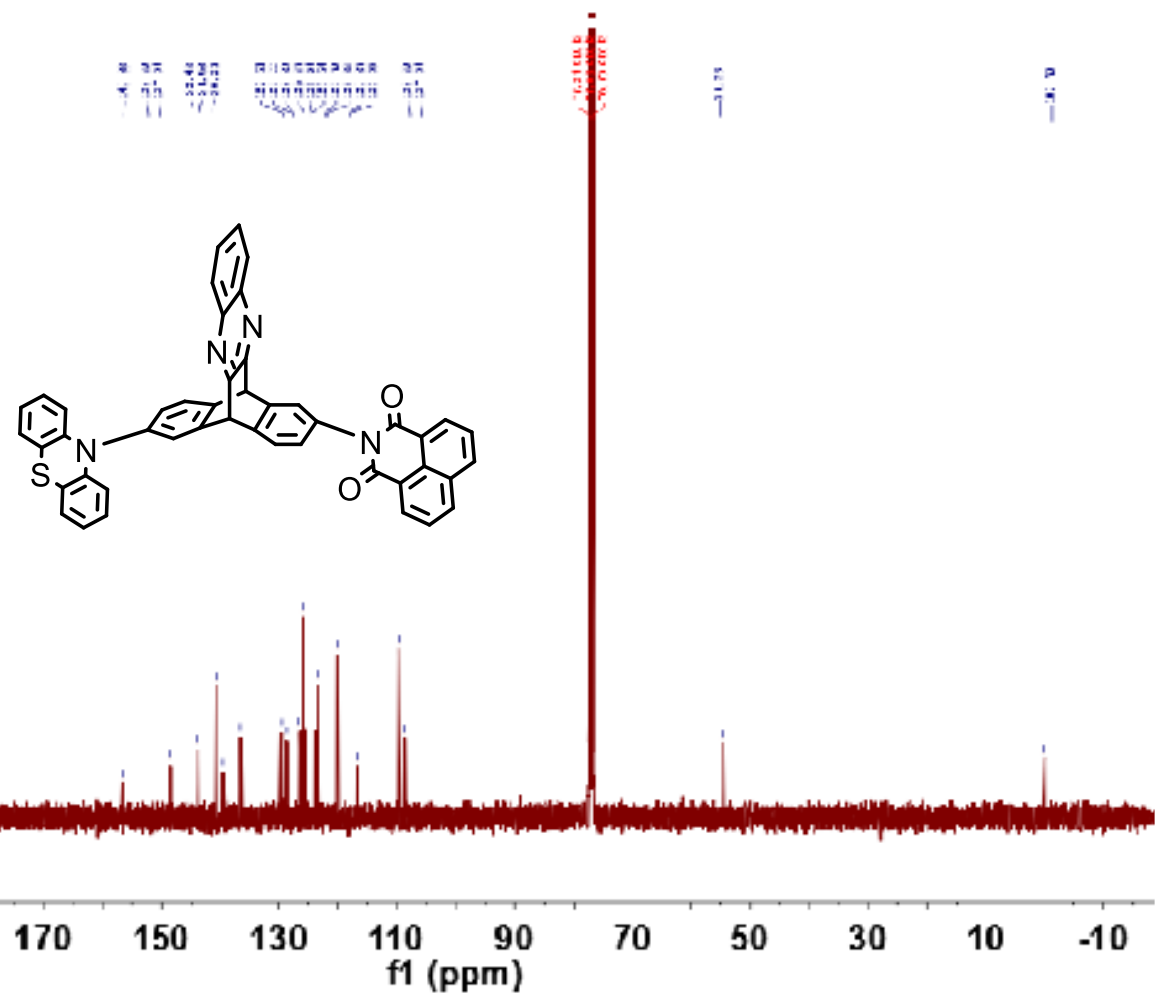

Figure S23. ${ }^{13} \mathrm{C}$ NMR $\left(101 \mathrm{MHz}, \mathrm{CDCl}_{3}\right)$ spectrum of Pz-Npa.

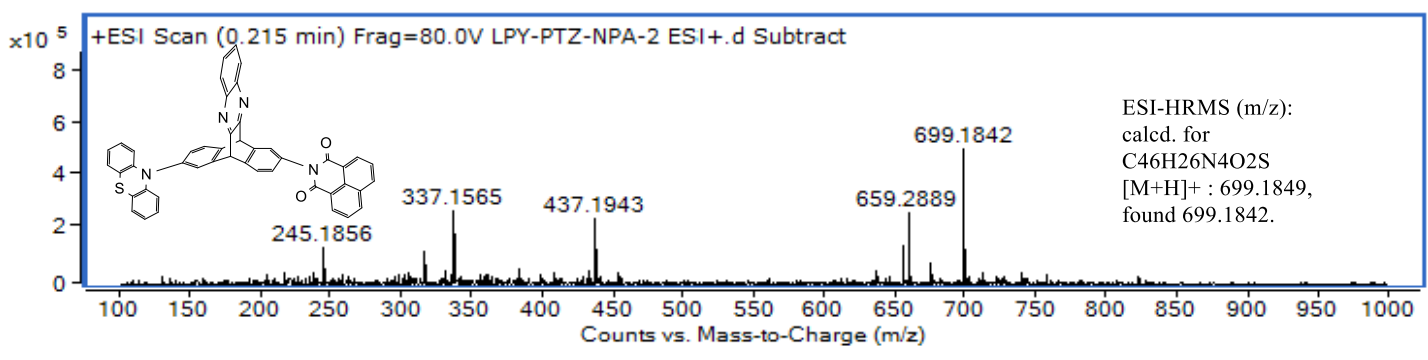

Figure S24. ESI-HRMS spectrum for Pz-Npa. 


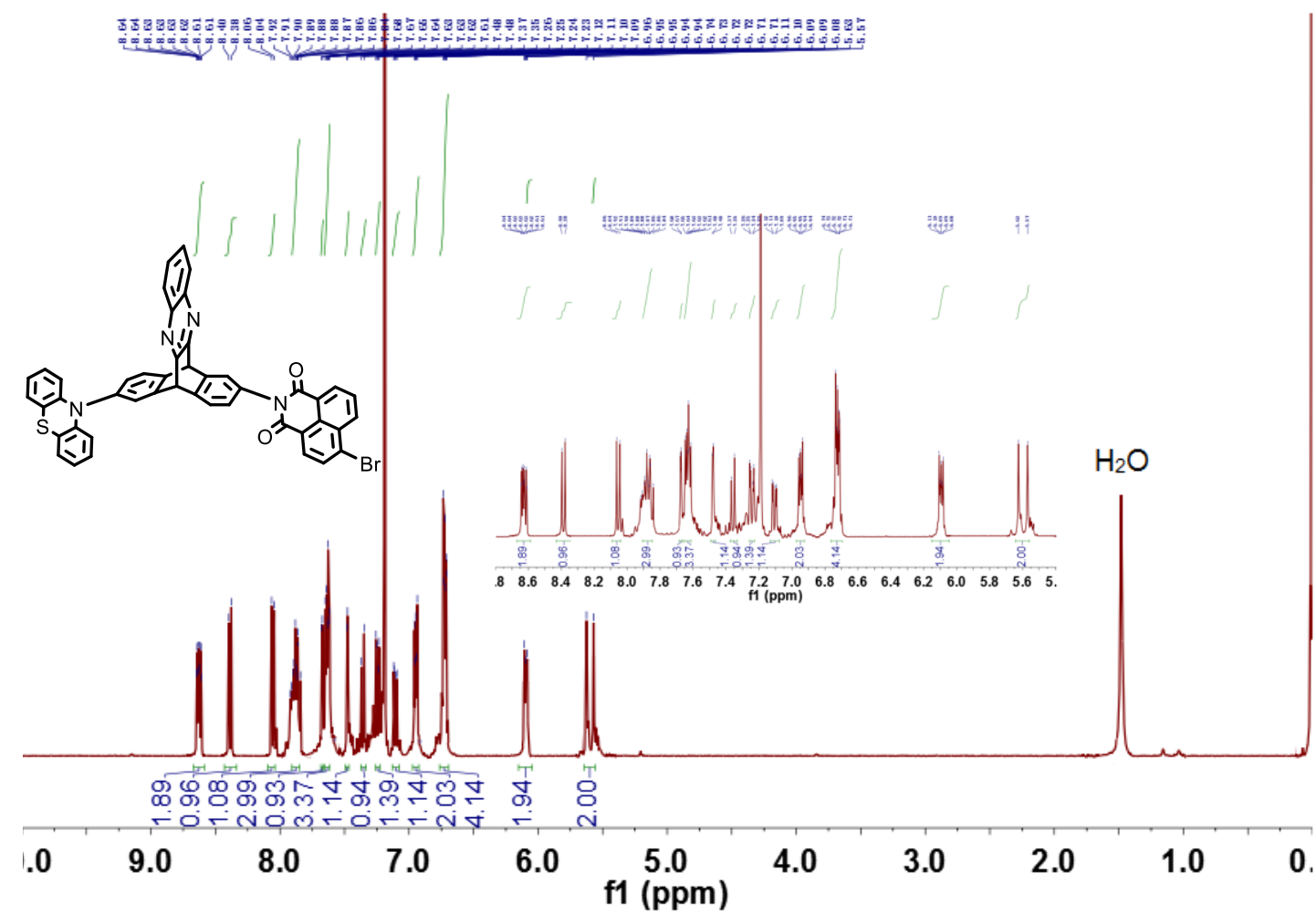

Figure S25. ${ }^{1} \mathrm{H}$ NMR $\left(400 \mathrm{MHz}, \mathrm{CDCl}_{3}\right)$ spectrum of Pz-NpaBr.

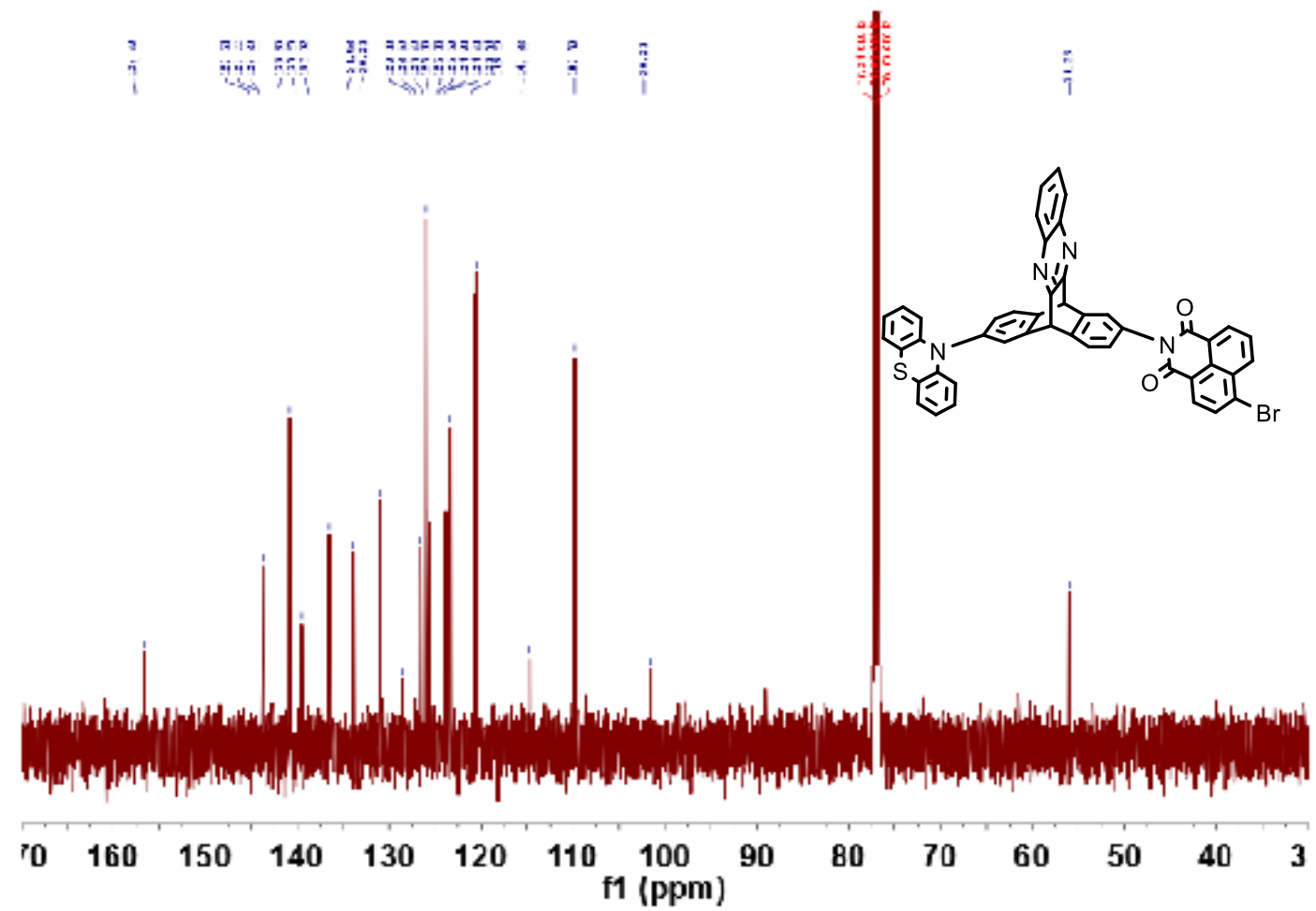

Figure S26. ${ }^{13} \mathrm{C}$ NMR $\left(101 \mathrm{MHz}, \mathrm{CDCl}_{3}\right)$ spectrum of Pz-NpaBr. 


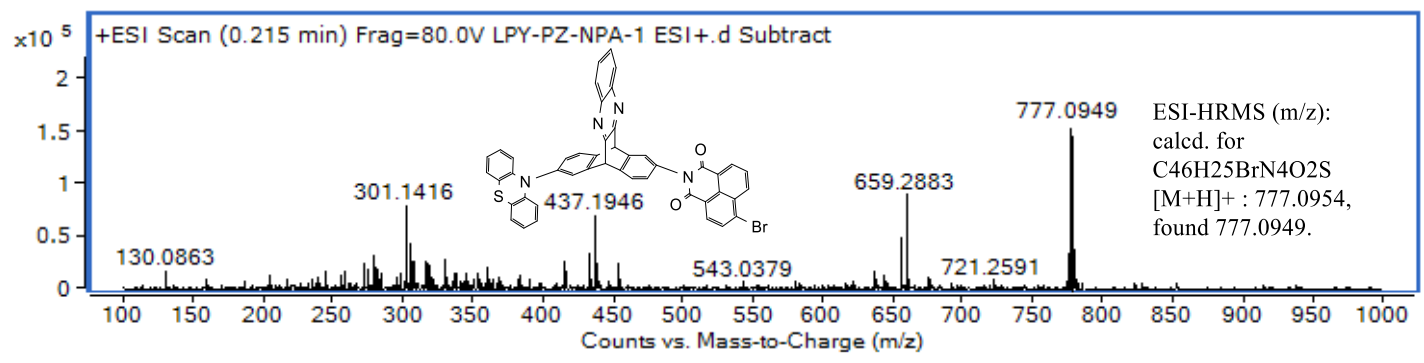

Figure S27. ESI-HRMS spectrum for Pz-NpaBr.

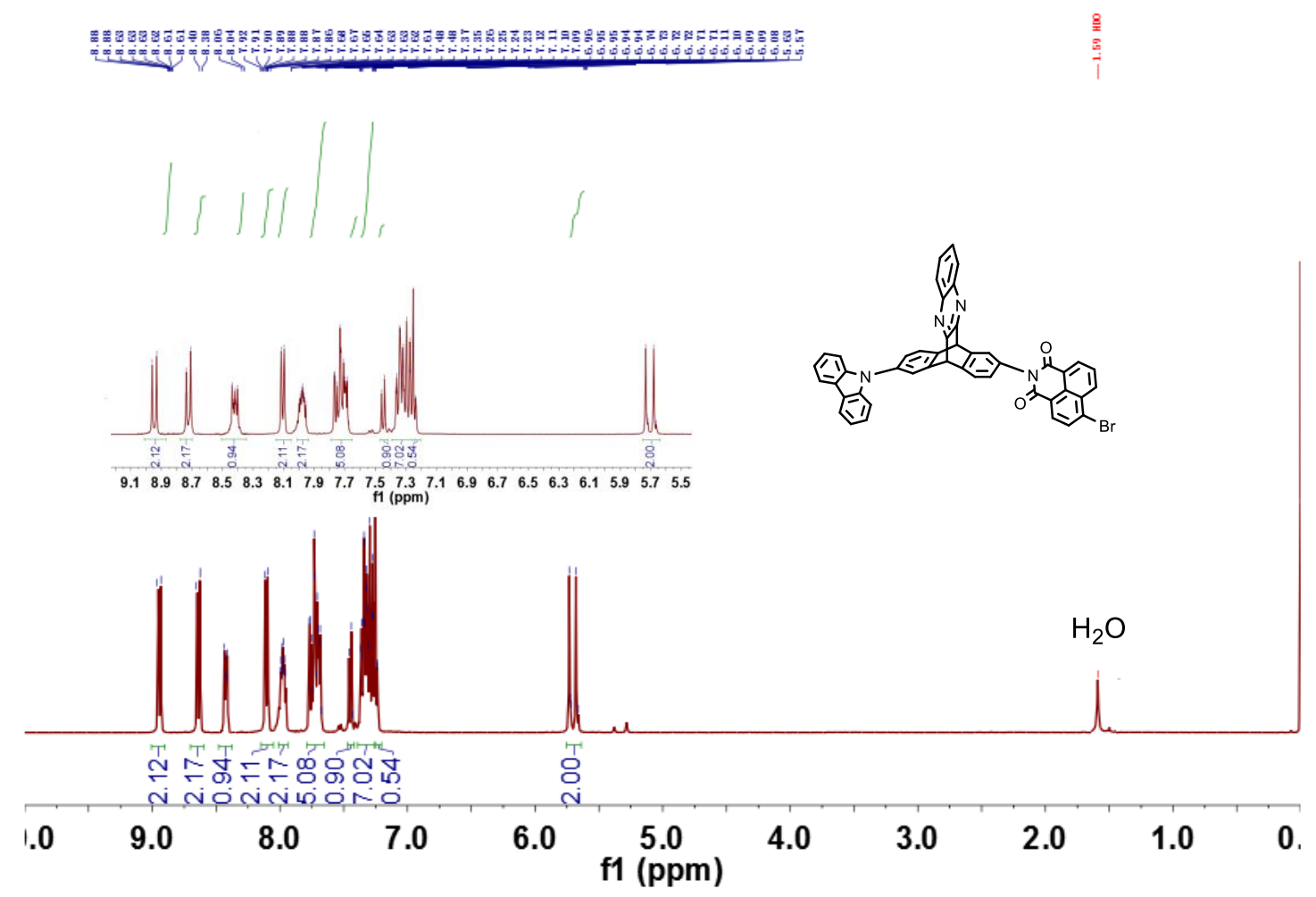

Figure S28. ${ }^{1} \mathrm{H}$ NMR (400 MHz, $\left.\mathrm{CDCl}_{3}\right)$ spectrum of $\mathbf{C z - N p a B r}$. 

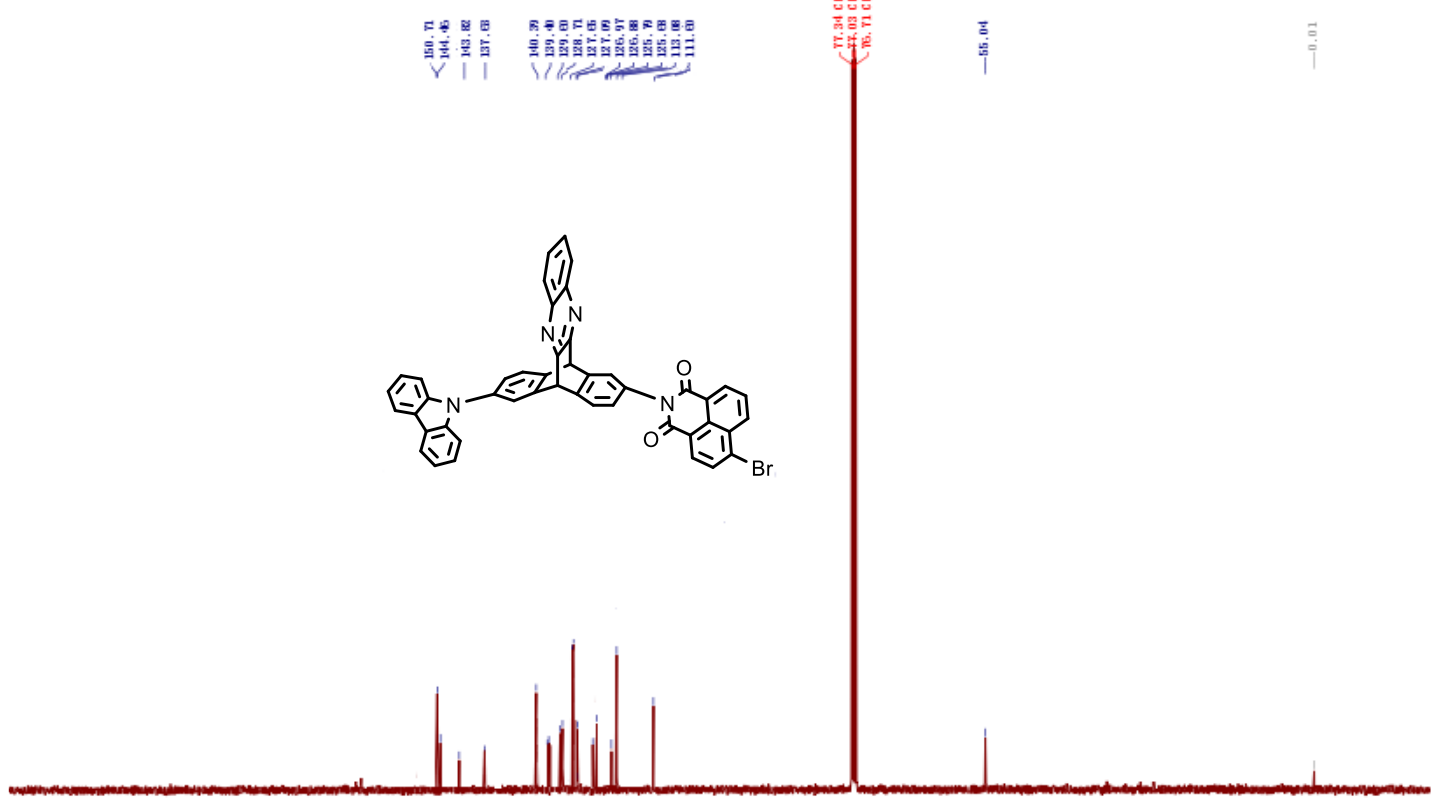

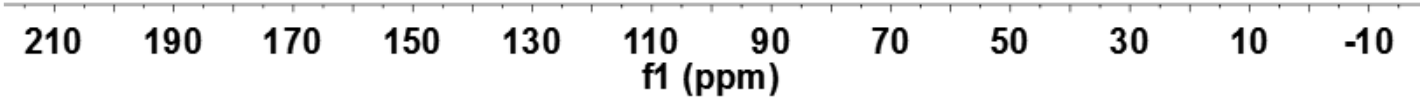

Figure S29. ${ }^{13} \mathrm{C}$ NMR $\left(101 \mathrm{MHz}, \mathrm{CDCl}_{3}\right)$ spectrum of $\mathbf{C z - N p a B r}$.

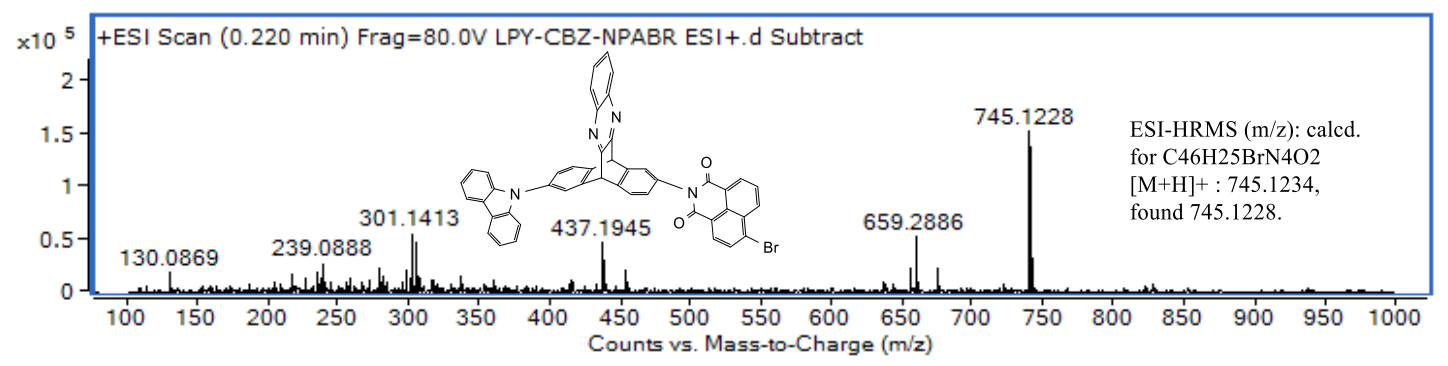

Figure S30. ESI-HRMS spectrum for $\mathbf{C z}-\mathbf{N p a B r}$. 


\section{XRD Measurements}
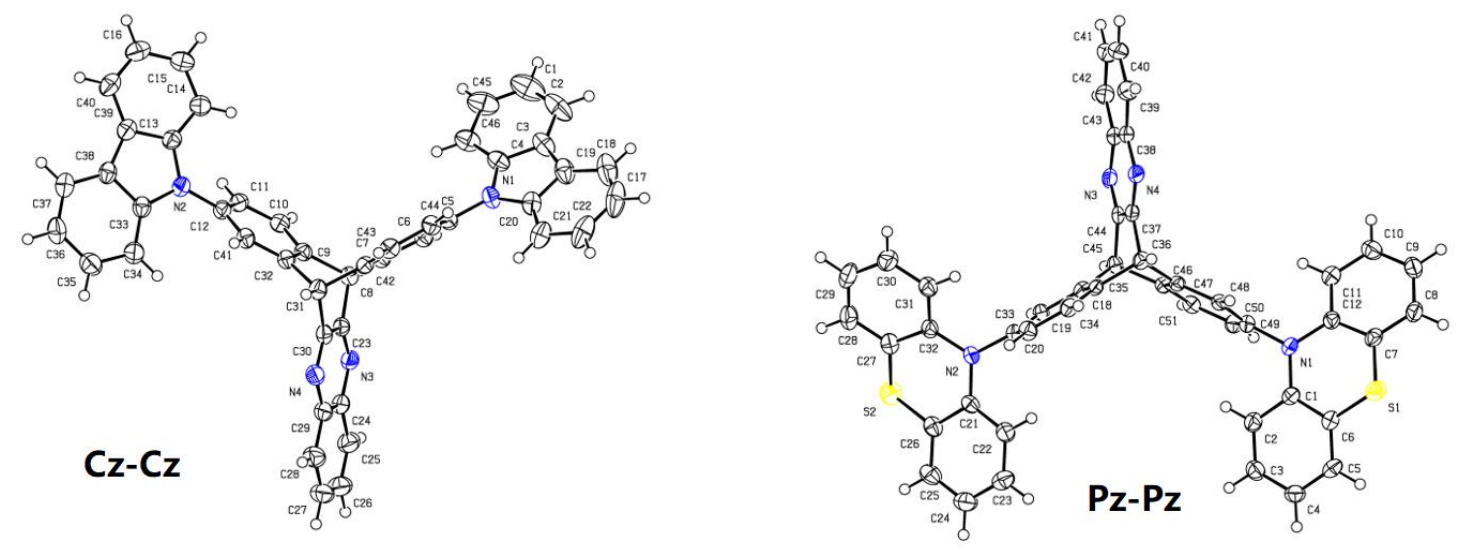

Figure S31. Single crystal $x$-ray structures of $\mathbf{C z}-\mathbf{C z}$ and $\mathbf{P z}-\mathbf{P z}$ (50\% thermal ellipsoids).

Table S1. Crystallographic data and refinements for $\mathbf{P z}-\mathbf{P z}$ and $\mathbf{C z}-\mathbf{C z}$.

\begin{tabular}{|c|c|c|}
\hline Identification code & $\mathbf{P z}-\mathbf{P z}$ & $\mathbf{C z}-\mathbf{C z}$ \\
\hline Empirical formula & $\mathrm{C}_{46} \mathrm{H}_{28} \mathrm{~N}_{4} \mathrm{~S}_{2}$ & $\mathrm{C}_{46} \mathrm{H}_{28} \mathrm{~N}_{4}$ \\
\hline Formula weight & 700.84 & 636.72 \\
\hline Temperature/K & 179.99 & 179.99 \\
\hline Crystal system & monoclinic & monoclinic \\
\hline Space group & $\mathrm{P} 2_{1} / \mathrm{c}$ & $\mathrm{P} 2_{1} / \mathrm{n}$ \\
\hline & $\mathrm{a}=11.3781(4) \AA$ & $\mathrm{a}=10.8827(4) \AA$ \\
& $\mathrm{b}=11.4044(4) \AA$ & $\mathrm{b}=25.7626(9) \AA$ \\
& $\mathrm{c}=28.9791(11) \AA$ & $\mathrm{c}=13.4803(5) \AA$ \\
& $\alpha=90^{\circ}$ & $\alpha=90^{\circ}$ \\
\hline Unit cell & $\beta=96.8230(10)^{\circ}$ & $\beta=98.6170(10)^{\circ}$ \\
& $\gamma=90^{\circ}$ & $\gamma=90^{\circ}$ \\
\hline Volume/ $\AA^{3}$ & $3733.7(2)$ & $3736.8(2)$ \\
\hline $\mathrm{Z}$ & 4 & 4 \\
\hline$\rho_{\text {calcg/cm }}{ }^{3}$ & 1.247 & 1.132 \\
\hline$\mu / \mathrm{mm}^{-1}$ & 0.181 & 0.067 \\
\hline $\mathrm{F}(000)$ & 1456.0 & 1328.0 \\
\hline Crystal size/mm & $0.26 \times 0.16 \times 0.08$ & $0.29 \times 0.21 \times 0.1$ \\
\hline & & \\
\hline & & \\
\hline
\end{tabular}




\begin{tabular}{|c|c|c|}
\hline Radiation & $\operatorname{MoK} \alpha(\lambda=0.71073)$ & $\operatorname{MoK} \alpha(\lambda=0.71073)$ \\
\hline $\begin{array}{c}2 \theta \text { range for data } \\
\text { collection }^{\circ}\end{array}$ & $4.312-55.13$ & $4.398-55.156$ \\
\hline Index ranges & $\begin{array}{c}-13 \leq \mathrm{h} \leq 14,-14 \leq \mathrm{k} \leq 14,-37 \leq 1 \leq \\
37\end{array}$ & $\begin{array}{c}-12 \leq \mathrm{h} \leq 14,-33 \leq \mathrm{k} \leq 33,-16 \leq 1 \leq \\
17\end{array}$ \\
\hline Reflections collected & 52098 & 53192 \\
\hline Independent reflections & $8609\left[\mathrm{R}_{\mathrm{int}}=0.0815, \mathrm{R}_{\text {sigma }}=0.0645\right]$ & $8611\left[\mathrm{R}_{\text {int }}=0.0725, \mathrm{R}_{\text {sigma }}=0.0615\right]$ \\
\hline $\begin{array}{l}\text { Data/restraints/ } \\
\text { parameters }\end{array}$ & $8609 / 0 / 469$ & $8611 / 0 / 451$ \\
\hline Goodness-of-fit on $\mathrm{F}^{2}$ & 1.043 & 1.038 \\
\hline $\begin{array}{l}\text { Final } \mathrm{R} \text { indexes }[\mathrm{I}>=2 \sigma \\
(\mathrm{I})]\end{array}$ & $\begin{aligned} \mathrm{R}_{1} & =0.0504 \\
\mathrm{wR}_{2} & =0.1233\end{aligned}$ & $\begin{aligned} \mathrm{R}_{1} & =0.0496 \\
\mathrm{wR}_{2} & =0.1171\end{aligned}$ \\
\hline $\begin{array}{l}\text { Final } \mathrm{R} \text { indexes [all } \\
\text { data] }\end{array}$ & $\mathrm{R}_{1}=0.0955, \mathrm{wR}_{2}=0.1403$ & $\mathrm{R}_{1}=0.0926, \mathrm{wR}_{2}=0.1327$ \\
\hline $\begin{array}{c}\text { Largest diff. } \\
\text { peak/hole }\left(\mathrm{e} . \AA^{-3}\right)\end{array}$ & $0.32 /-0.30$ & $0.18 /-0.23$ \\
\hline
\end{tabular}

Table S2. Significant dihedral angles for $\mathbf{P z}-\mathbf{P z}$ and $\mathbf{C z}-\mathbf{C z}$.

\begin{tabular}{cccc}
\hline & Plane A & Plane B & Angle $^{\circ}$ \\
\hline C8-C7-C42-C31 & C30-C23-C31-C8 & 119.92 \\
Cz-Cz & C30-C23-C31-C8 & C9-C32-C8-C31 & 120.27 \\
& C9-C32-C8-C31 & C8-C7-C42-C31 & 119.81 \\
& C11-C10-C9-C32-C41-C12 & C33-C38-C39-C13-N2 & 49.85 \\
C43-C44-C5-C6-C7-C42 & C3-C4-N1-C20-C19 & 46.94 \\
\hline C44-C37-C36-C45 & C35-C36-C45-18 & 119.92 \\
& C35-C36-C45-18 & C36-C45-C46-C47 & 121.07 \\
& C36-C45-C46-C47 & C44-C37-C36-C45 & 119.81 \\
& C1-C6-C5-C4-C3-C2 & C10-C9-C8-C7-C11-C12 & 151.11 \\
& C29-C30-C31-C32-C27-C28 & C23-C24-C25-C26-C21-C22 & 150.55 \\
C23-C24-C25-C26-C21-C22 & C33-C34-C35-C18-C19-C20 & 71.30 \\
& C29-C30-C31-C32-C27-C28 & C33-C34-C35-C18-C19-C20 & 79.42 \\
C10-C9-C8-C7-C11-C12 & C51-C46-C47-C48-C49-C50 & 82.01 \\
& C1-C6-C5-C4-C3-C2 & C51-C46-C47-C48-C49-C50 & 69.13 \\
\hline
\end{tabular}




\section{Electrochemistry Examination}
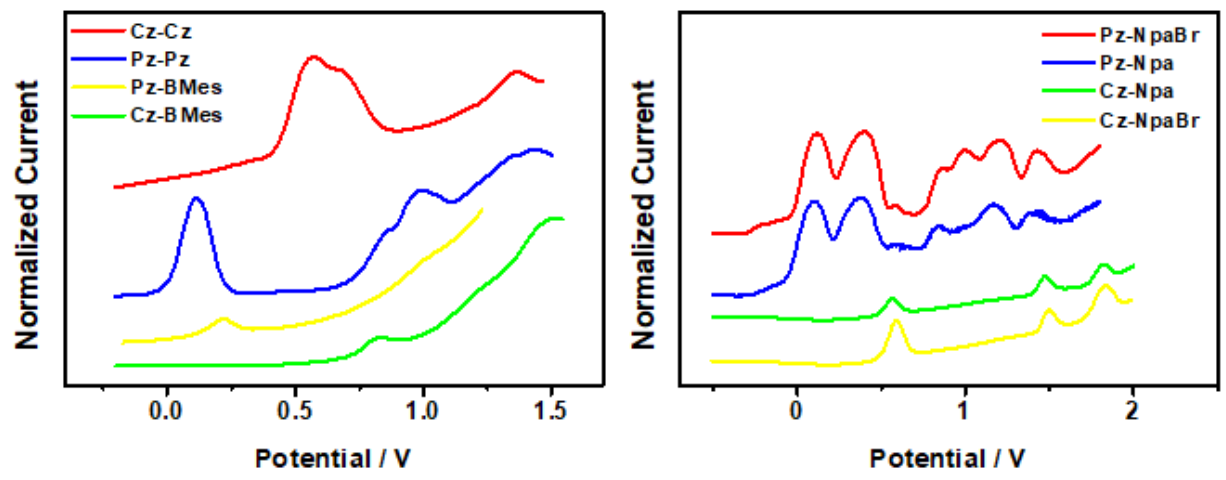

Figure S32. DPV scans of oxidation curves recorded in $\mathrm{CH}_{2} \mathrm{Cl}_{2}$ for triptycene derivatives with $\left[\mathrm{NBu}_{4}\right] \mathrm{PF}_{6}$ as supporting electrolyte $(c=0.1 \mathrm{M})$ at $v=100 \mathrm{mV} / \mathrm{s}, v s$ $\mathrm{Fc} / \mathrm{Fc}^{+}$.

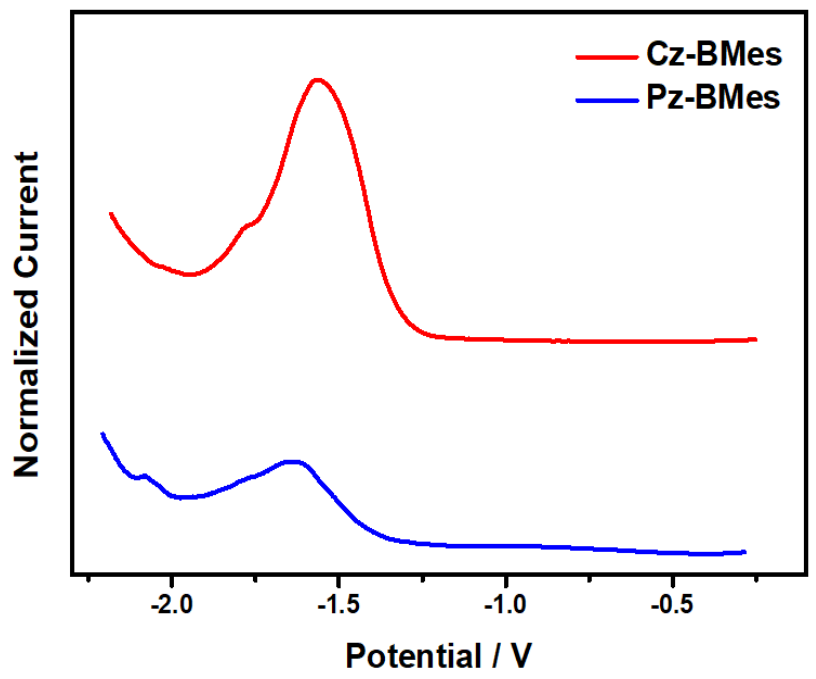

Figure S33. DPV scans of reduction curves recorded in THF for Cz-BMes and PzBMes with $\left[\mathrm{NBu}_{4}\right] \mathrm{PF}_{6}$ as supporting electrolyte $(c=0.1 \mathrm{M})$ at $v=100 \mathrm{mV} / \mathrm{s}, v s \mathrm{Fc} / \mathrm{Fc}^{+}$. 
Table S3. Differential pulse voltammetry (DPV) data and comparison with DFTcalculated results of triptycene derivatives.

\begin{tabular}{lcccccc}
\hline & $\begin{array}{l}E_{\text {оx }}{ }^{a} \\
(\mathrm{~V})\end{array}$ & $\begin{array}{l}E_{\mathrm{HOMO}^{b}} \\
(\mathrm{eV})\end{array}$ & $\begin{array}{l}E_{\mathrm{HOMO}^{c}} \\
(\mathrm{eV})\end{array}$ & $\begin{array}{l}E_{\mathrm{LUMO}^{c}} \\
(\mathrm{eV})\end{array}$ & $\begin{array}{l}E_{\mathrm{g}}{ }^{c} \\
(\mathrm{eV})\end{array}$ & $\begin{array}{l}E_{\mathrm{g}}{ }^{d} \\
(\mathrm{eV})\end{array}$ \\
\hline Cz-Cz & 0.58 & -5.38 & -5.39 & -1.88 & 3.51 & 3.53 \\
Cz-BMes & 0.83 & -5.63 & -5.35 & -1.85 & 3.50 & 3.45 \\
Cz-Npa & 0.56 & -5.36 & -5.27 & -2.48 & 2.79 & 2.90 \\
Cz-NpaBr & 0.59 & -5.39 & -5.30 & -2.68 & 2.62 & 2.68 \\
Pz-Pz & 0.12 & -4.92 & -5.01 & -1.92 & 3.09 & 3.33 \\
Pz-BMes & 0.22 & -5.02 & -4.95 & -1.86 & 3.09 & 3.45 \\
Pz-Npa & 0.10 & -4.90 & -4.87 & -2.50 & 2.37 & 2.65 \\
Pz-NpaBr & 0.12 & -4.92 & -4.90 & -2.70 & 2.20 & 2.44 \\
\hline
\end{tabular}

${ }^{a}$ First oxidation data from DPV recorded in $\mathrm{CH}_{2} \mathrm{Cl}_{2}\left(c=1.0 \times 10^{-5} \mathrm{M}\right)$ with $\left[\mathrm{NBu}_{4}\right] \mathrm{PF}_{6}$ as electrolyte. ${ }^{b} E_{\mathrm{HOMO}}=-4.8-E_{\mathrm{Ox}} ;{ }^{c}$ Obtained by DFT calculations (B3LYP, 6-31G*). ${ }^{d}$ Calculated from the experimental absorption onset.

\section{Optical Property Examination}

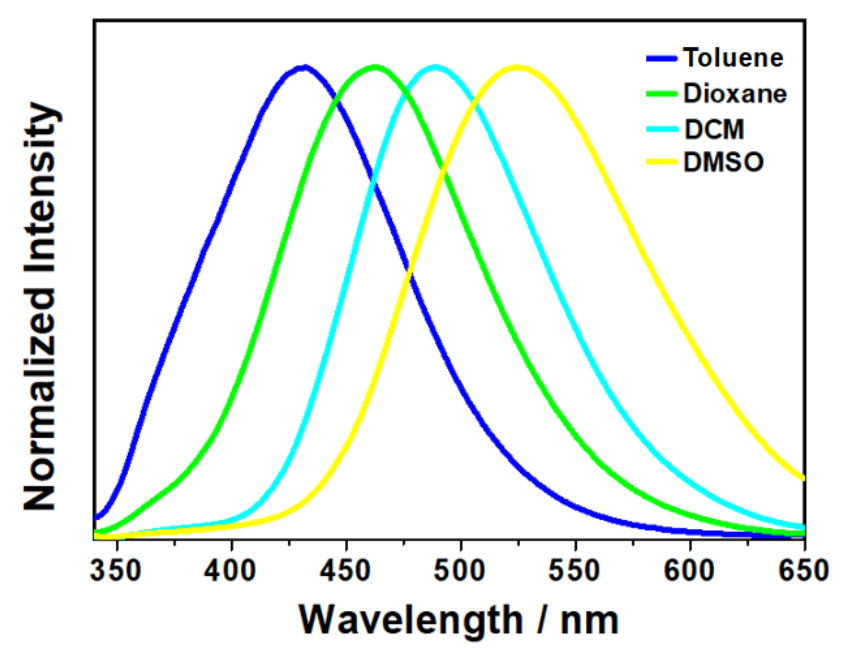

Figure S34. Solvatochromic emissions of Cz-BMes $\left(c=1.0 \times 10^{-5} \mathrm{M}\right)$ in different solvents of polarity. 

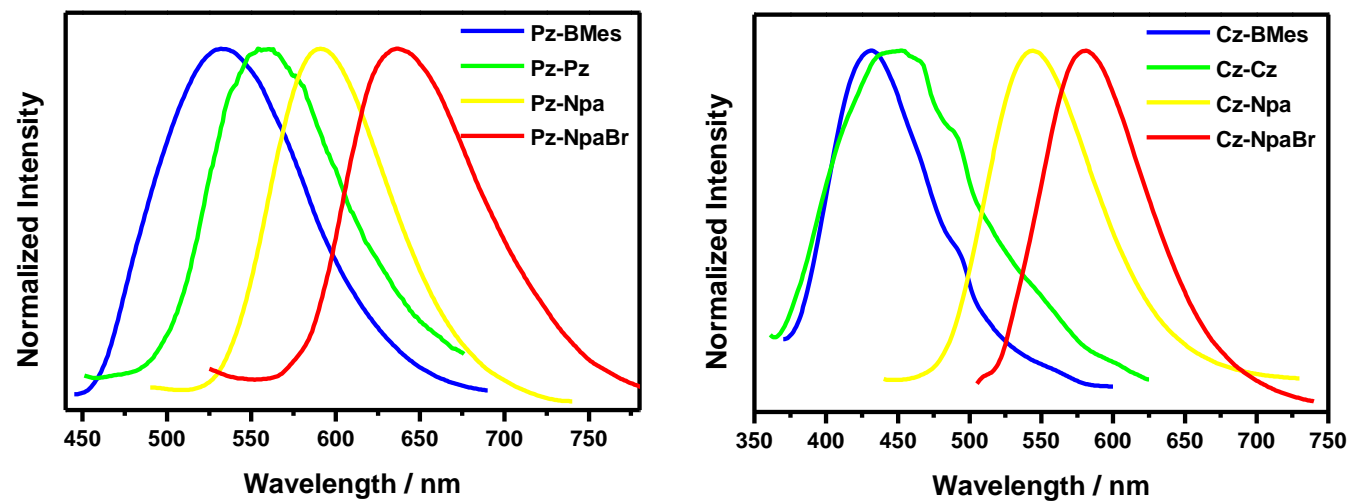

Figure S35. Emission spectra in thin film state.
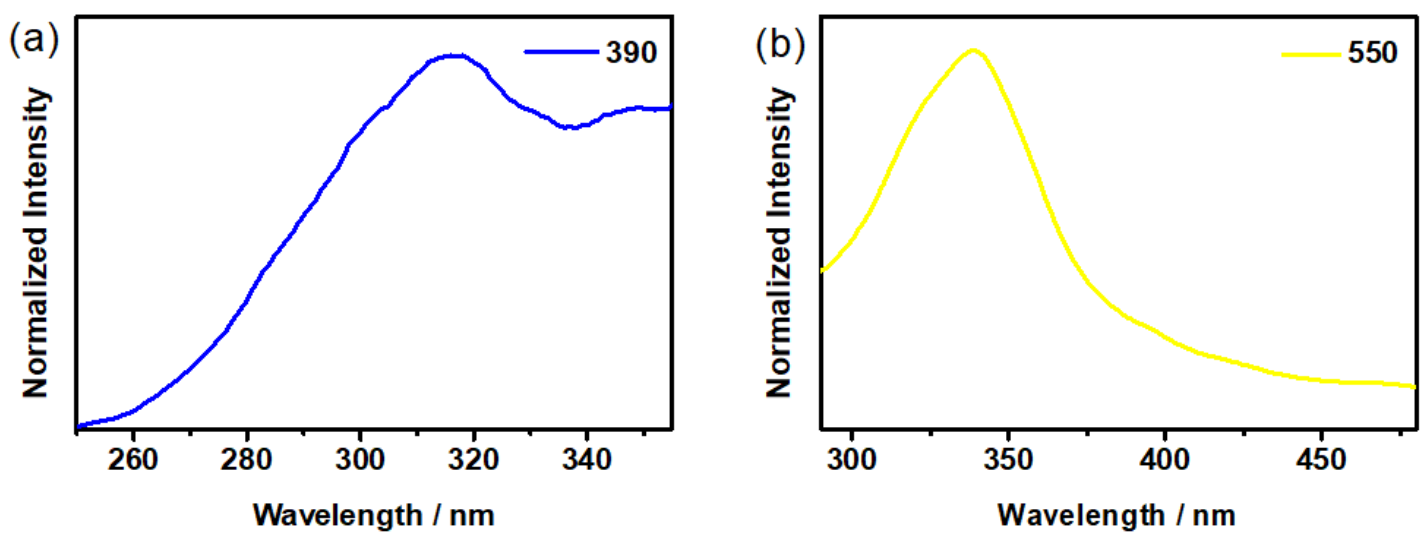

Figure S36. Excitation spectra of Pz-Pz in $\mathrm{H}_{2} \mathrm{O} / \mathrm{THF}\left(f_{\mathrm{w}}=90 \%\right)$ at $\lambda_{\mathrm{em}}=390 \mathrm{~nm}$ (a) and $\lambda_{\mathrm{em}}=550 \mathrm{~nm}(\mathrm{~b})$. 


\section{DFT and TD-DFT Computations}

DFT and TD-DFT calculations were performed using the Gaussian 09 suite of programs. ${ }^{[1]}$ Geometry optimizations and vertical excitations of all compounds were obtained at the B3LYP/6-31G* and B3LYP/6-311G** level of theory. ${ }^{[2,3]}$
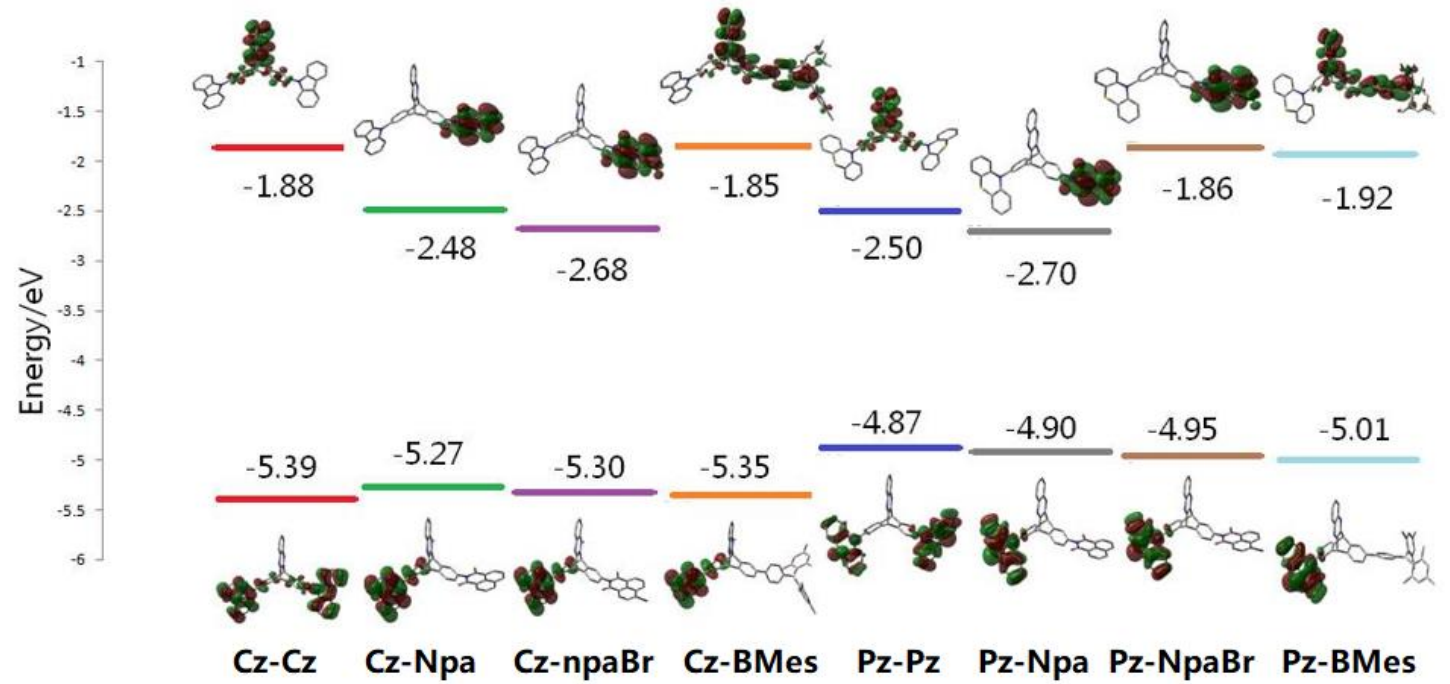

Figure S37. DFT optimized structures and energy levels of HOMOs and LUMOs (B3LYP, 6-31G*).

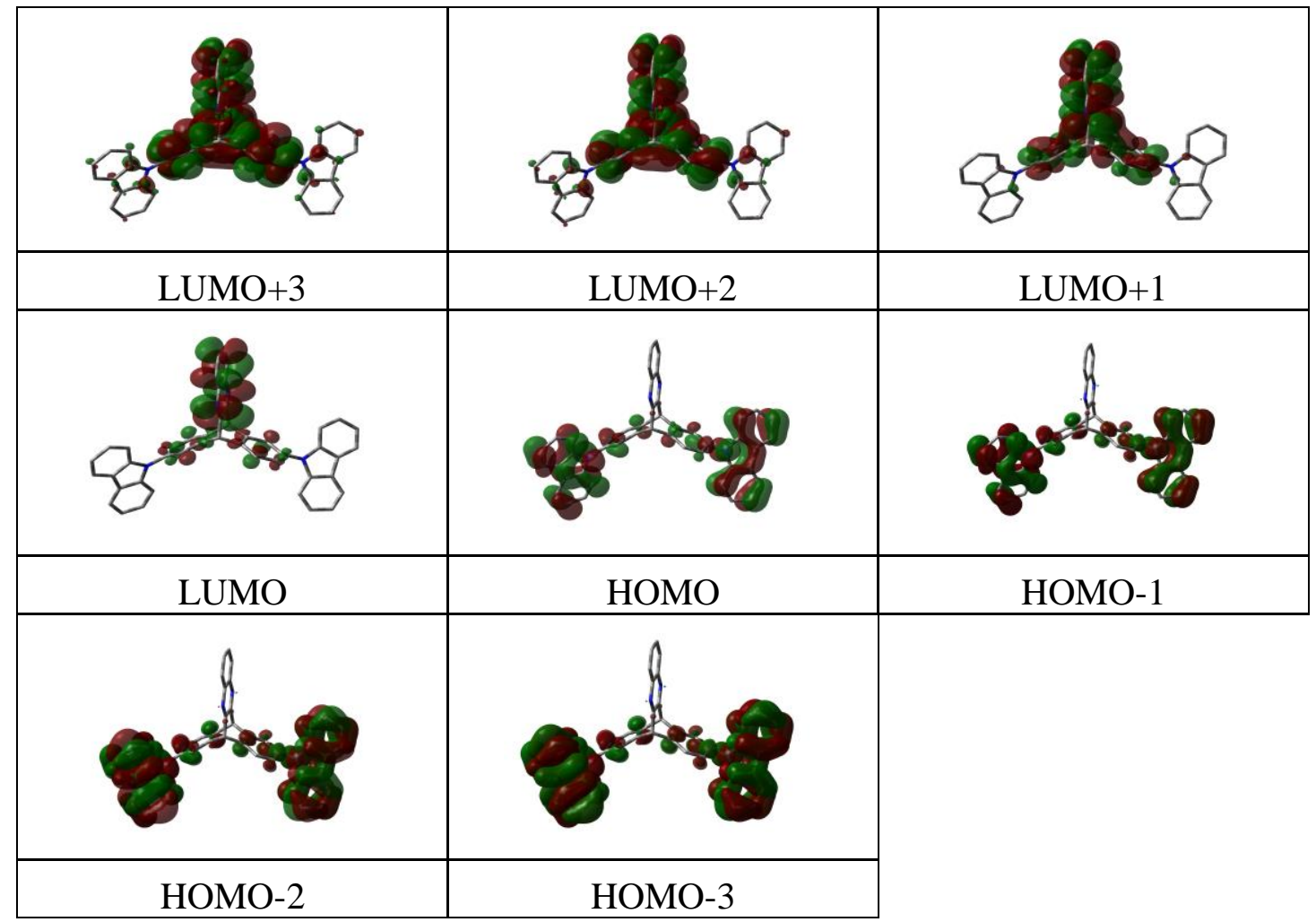

Figure S38. Primary orbitals contributing to the DFT calculated transitions of $\mathbf{C z}-\mathbf{C z}$ (iso $=0.02, \mathrm{~B} 3 \mathrm{LYP} / 6-31 \mathrm{G}^{*}$ ). 


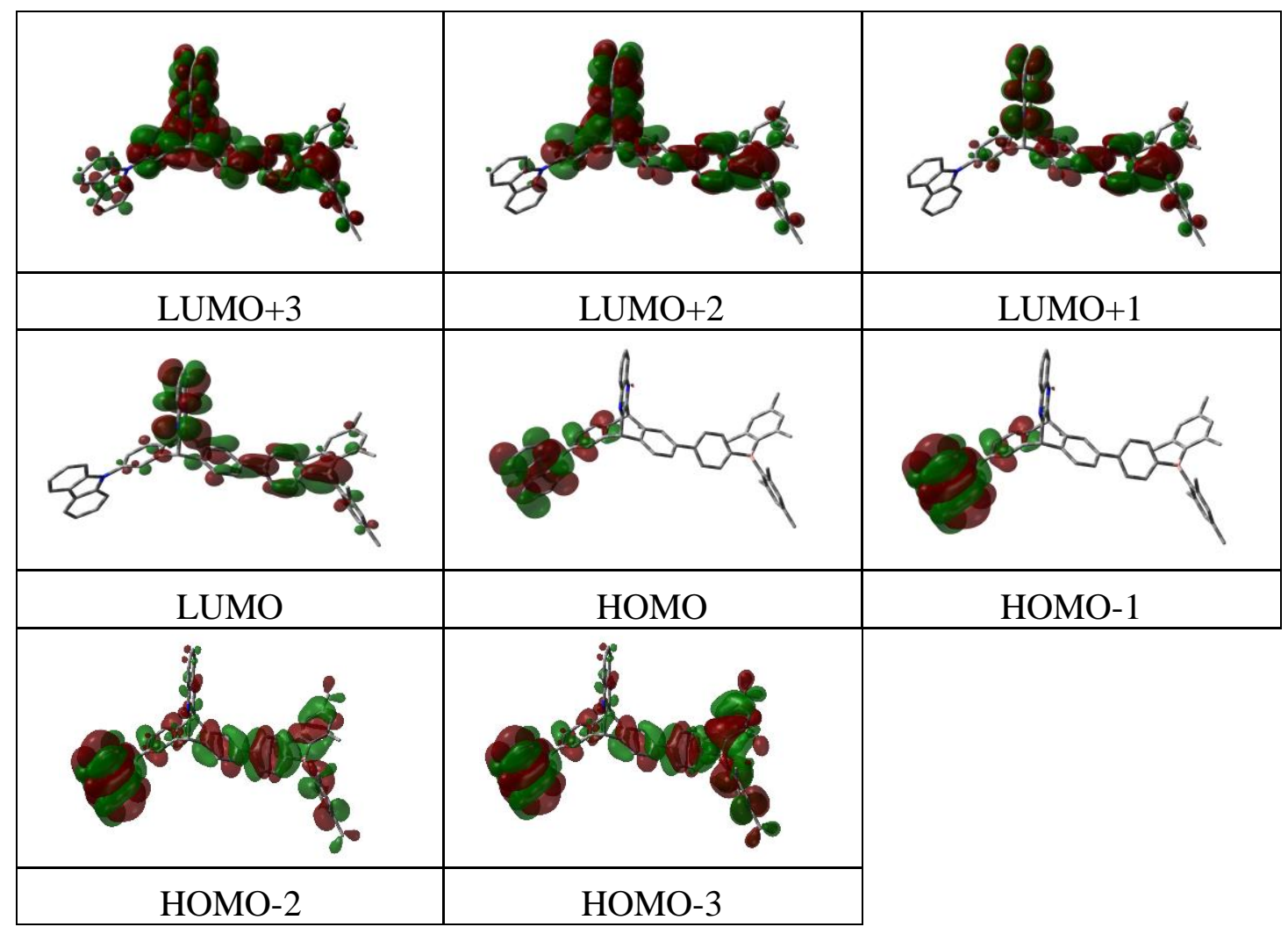

Figure S39. Primary orbitals contributing to the DFT calculated transitions of CzBMes (iso $=0.02, \mathrm{~B} 3 \mathrm{LYP} / 6-31 \mathrm{G}^{*}$ ).

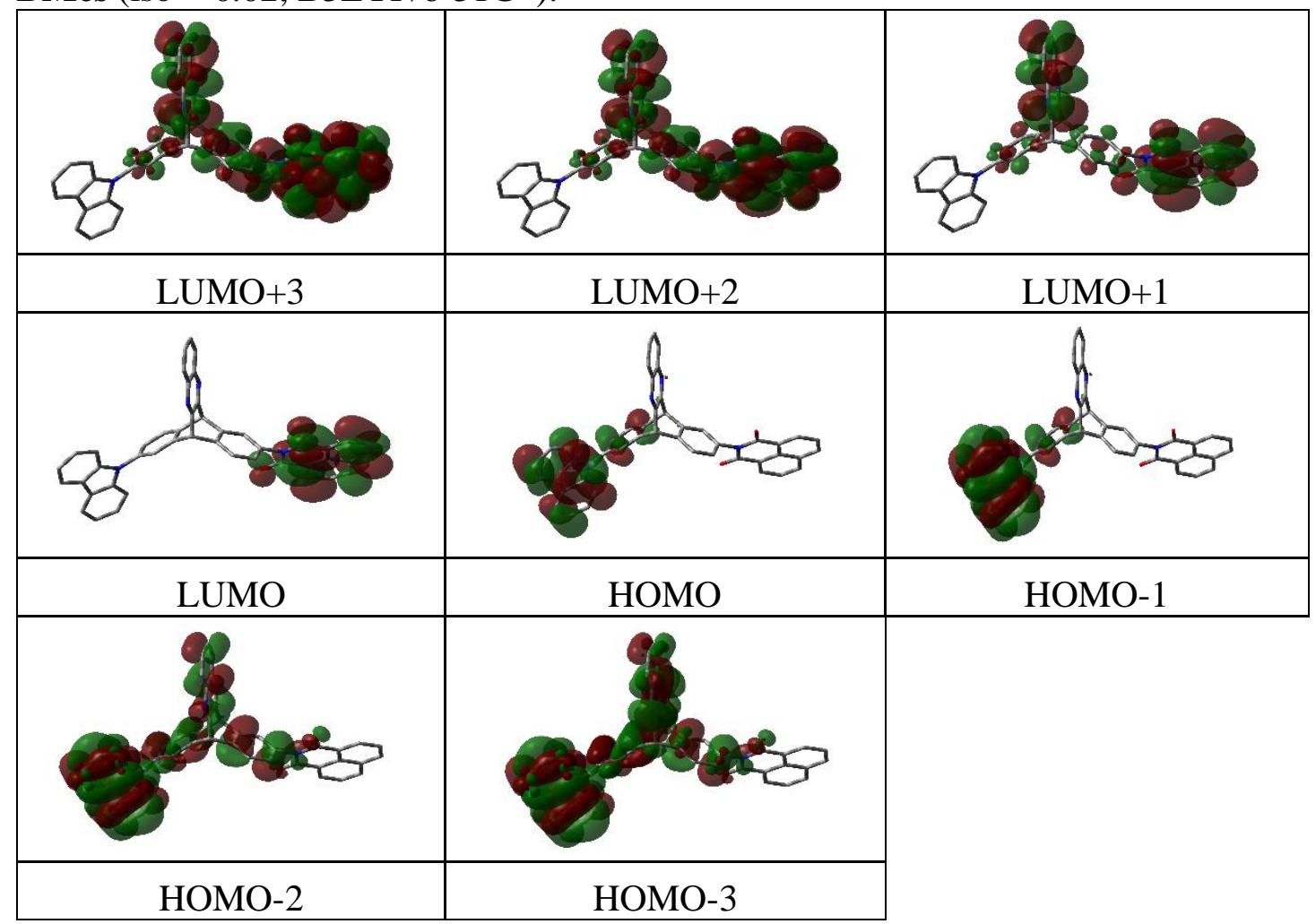

Figure S40. Primary orbitals contributing to the DFT calculated transitions of $\mathbf{C z}-\mathbf{N p a}$ (iso $=0.02, \mathrm{~B} 3 \mathrm{LYP} / 6-31 \mathrm{G}^{*}$ ). 


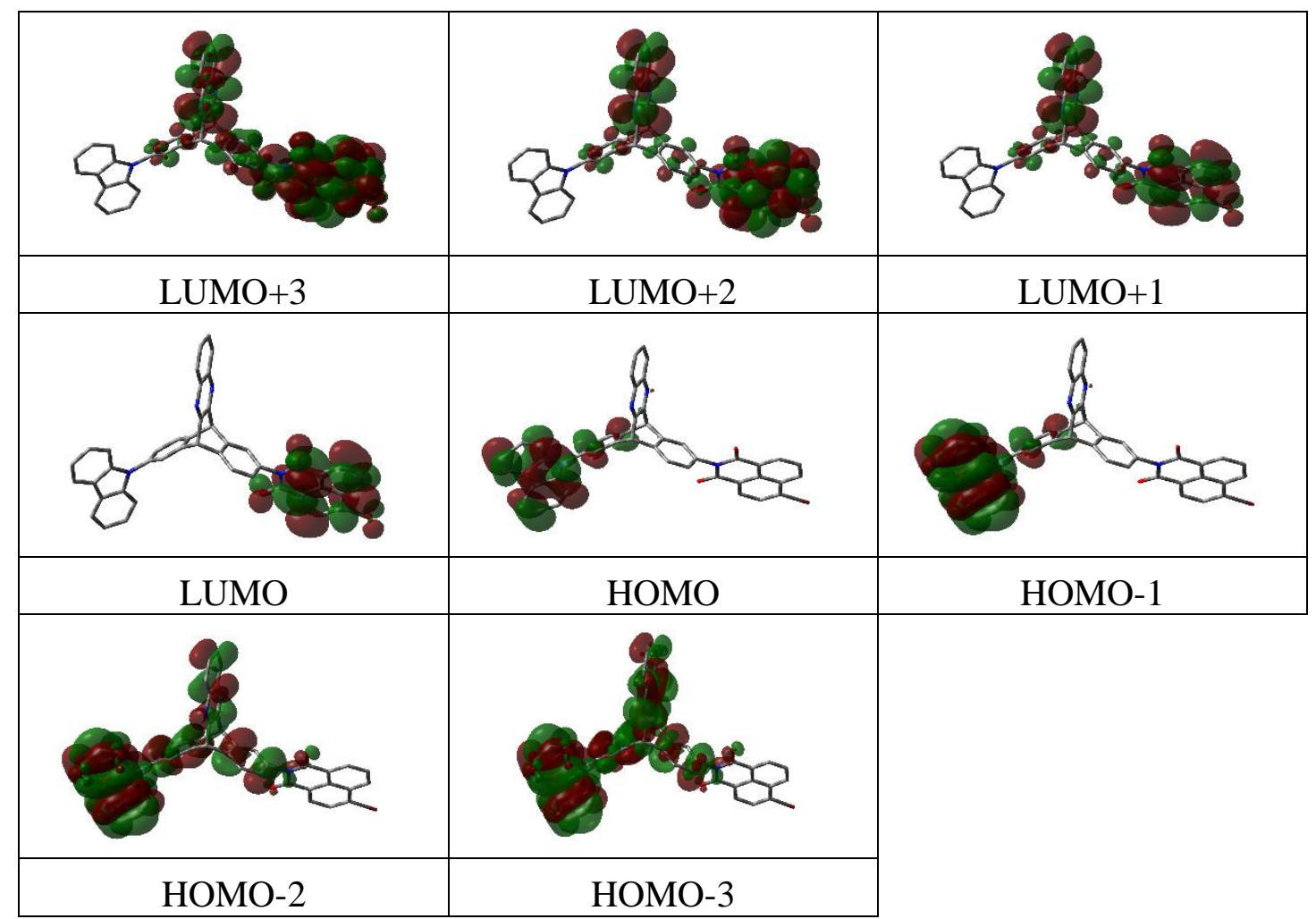

Figure S41. Primary orbitals contributing to the DFT calculated transitions of $\mathbf{C z}-$ NpaBr (iso $=0.02$, B3LYP/6-31G*).

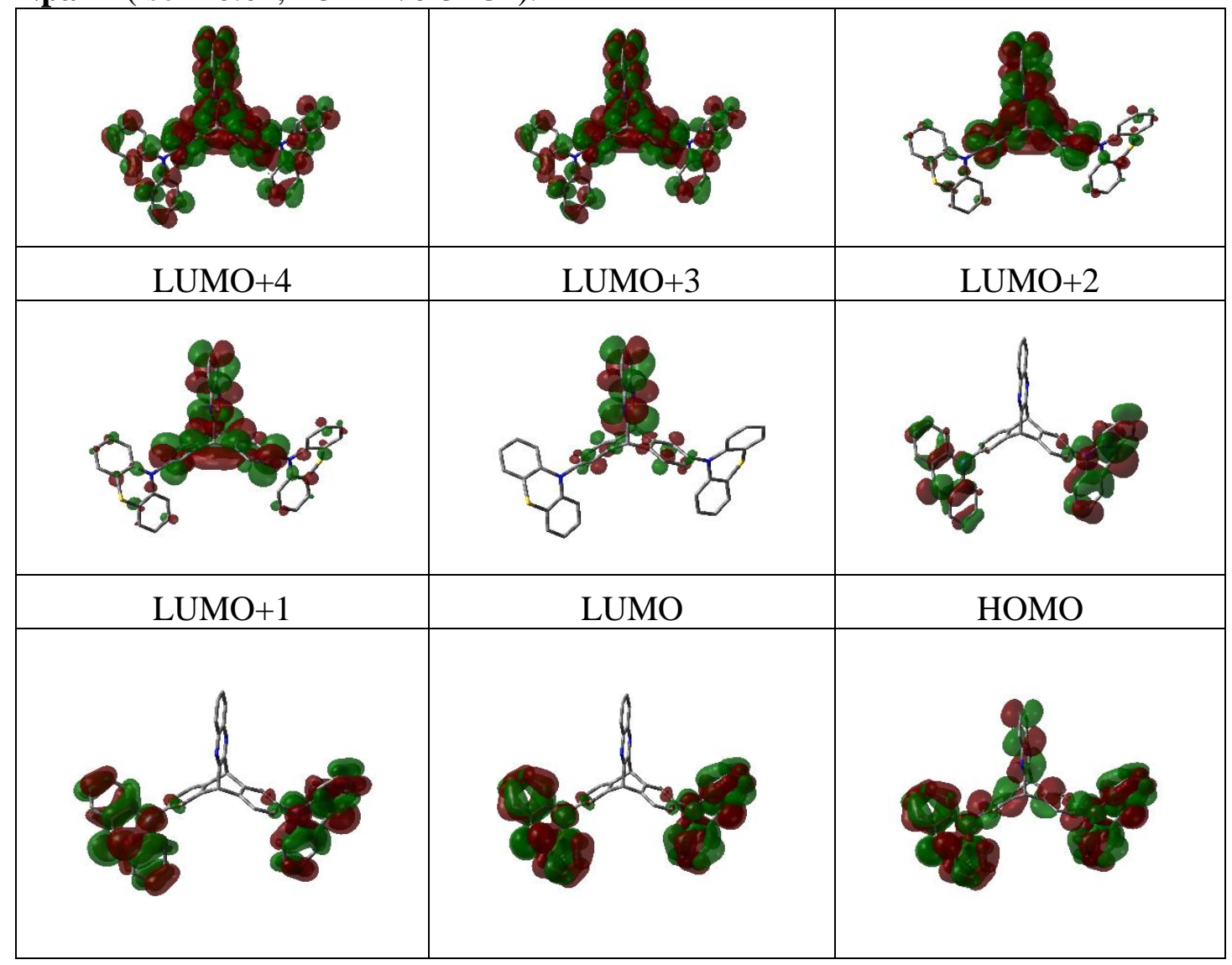


HOMO-1

HOMO-2

HOMO-3

Figure S42. Primary orbitals contributing to the DFT calculated transitions of Pz-Pz (iso $=0.02, \mathrm{~B} 3 \mathrm{LYP} / 6-31 \mathrm{G}^{*}$ ).

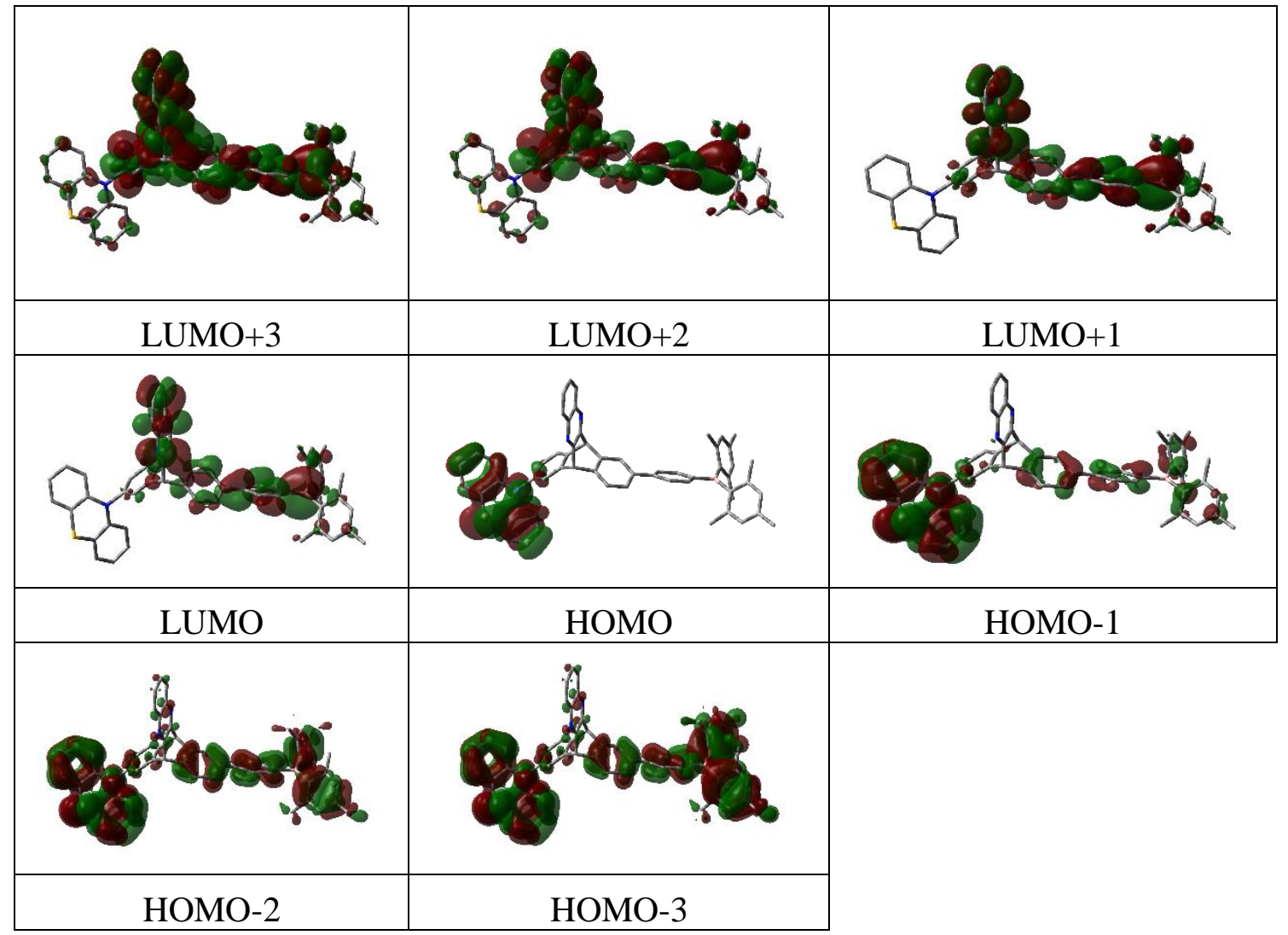

Figure S43. Primary orbitals contributing to the DFT calculated transitions of Pz-BMes (iso $\left.=0.02, \mathrm{~B} 3 \mathrm{LYP} / 6-31 \mathrm{G}^{*}\right)$.

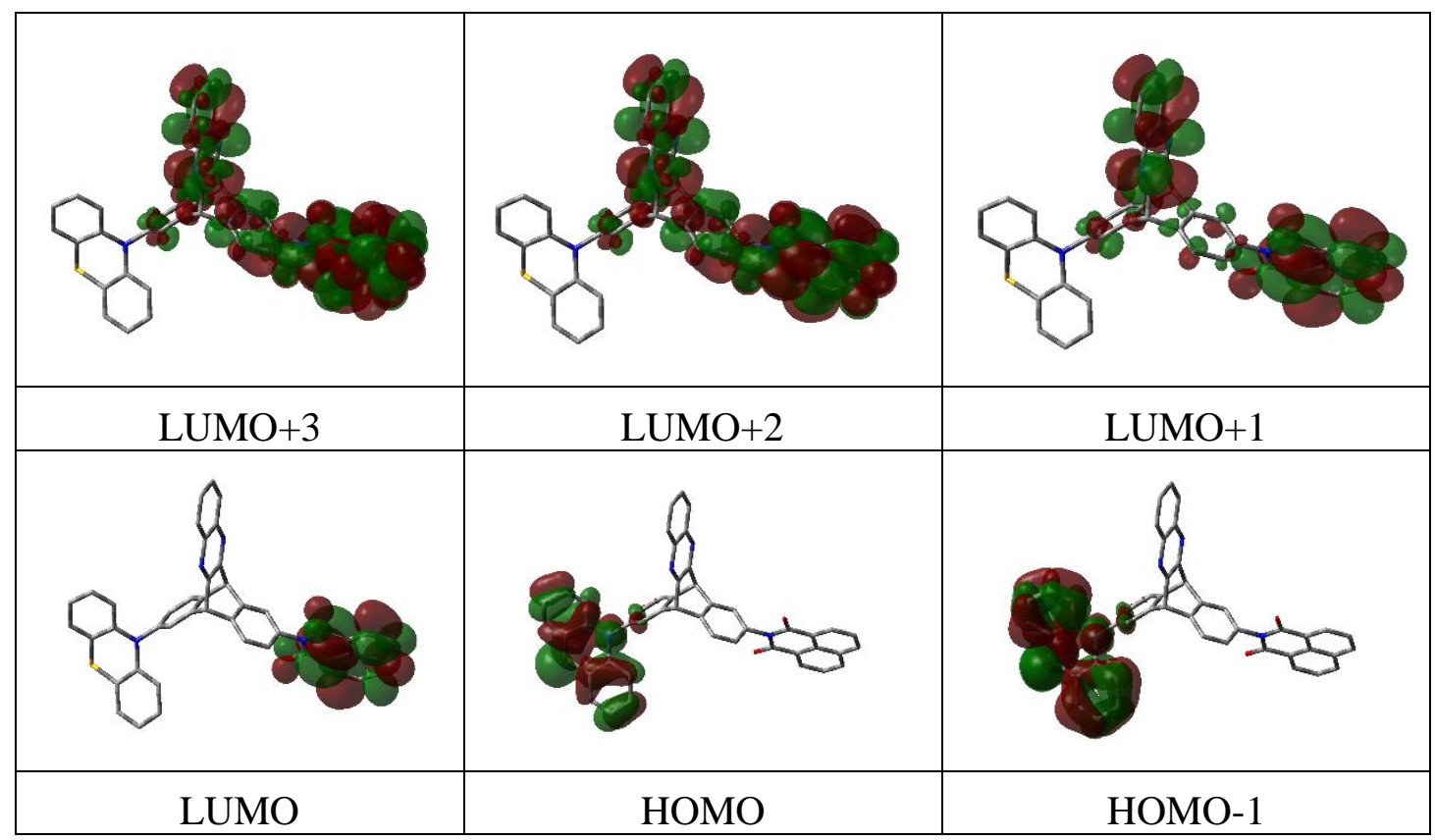




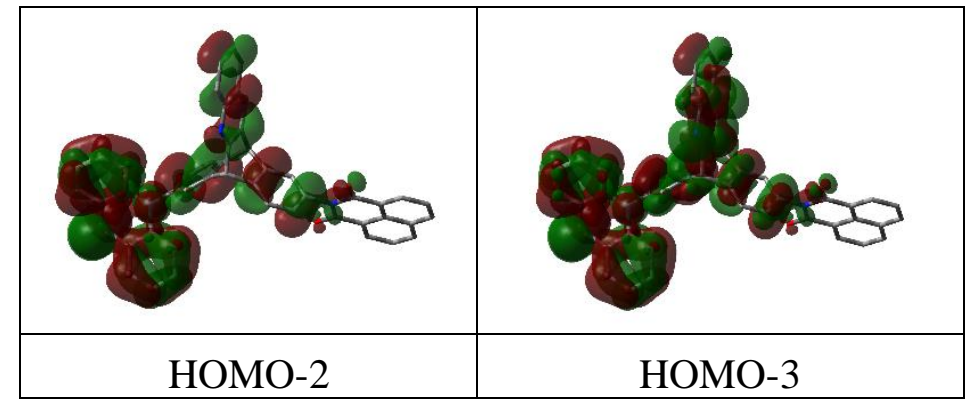

Figure S44. Primary orbitals contributing to the DFT calculated transitions of Pz-Npa (iso $\left.=0.02, \mathrm{~B} 3 \mathrm{LYP} / 6-31 \mathrm{G}^{*}\right)$.

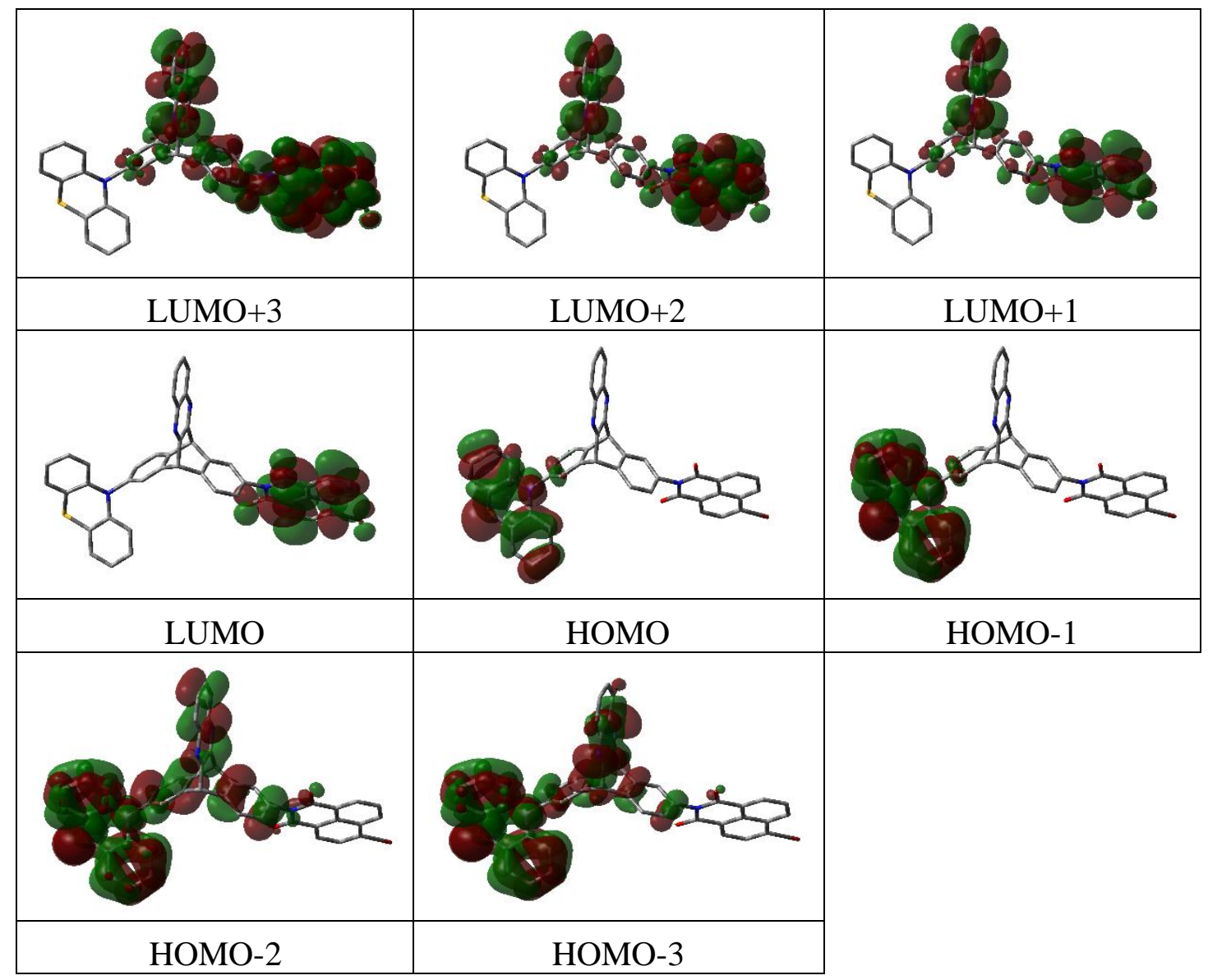

Figure S45. Primary orbitals contributing to the DFT calculated transitions of PzNpaBr (iso $=0.02$, B3LYP/6-31G*).

Table S4. Summary of the DFT and TD-DFT calculations.

\begin{tabular}{cccccccc}
\hline$E_{\mathrm{HOMO}^{a}}$ & $E_{\mathrm{LUMO}^{a}}$ & $E_{\mathrm{gap}}{ }^{b}$ & $E_{\mathrm{TD}^{-\mathrm{DFT}}}{ }^{c}$ & $E_{\mathrm{gap}(\mathrm{opt})^{d}}$ & $E_{\mathrm{HOMO}^{e}}$ & $E_{\mathrm{LUMO}^{e}}$ & $E_{\mathrm{gap}^{f}}$ \\
$(\mathrm{eV})$ & $(\mathrm{eV})$ & $(\mathrm{eV})$ & $(\mathrm{eV} / \mathrm{nm})$ & $(\mathrm{eV})$ & $(\mathrm{eV})$ & $(\mathrm{eV})$ & $(\mathrm{eV})$ \\
\hline
\end{tabular}




\begin{tabular}{lcccccccc} 
Cz-Cz & -5.39 & -1.88 & 3.51 & $3.10 / 400$ & 3.53 & -6.68 & -0.62 & 6.06 \\
Cz-Npa & -5.27 & -2.48 & 2.79 & $2.54 / 488$ & 2.90 & -6.56 & -1.28 & 5.28 \\
Cz-NpaBr & -5.30 & -2.68 & 2.62 & $2.37 / 522$ & 2.68 & -6.59 & -1.50 & 5.09 \\
Cz-BMes & -5.35 & -1.85 & 3.50 & $3.12 / 397$ & 3.45 & -6.64 & -0.62 & 6.02 \\
Pz-Npa & -4.87 & -2.50 & 2.37 & $2.11 / 586$ & 2.65 & -6.23 & -1.30 & 4.93 \\
Pz-NpaBr & -4.90 & -2.70 & 2.20 & $1.95 / 637$ & 2.44 & -6.26 & -1.52 & 4.74 \\
Pz-BMes & -4.95 & -1.86 & 3.09 & $2.73 / 453$ & 3.45 & -6.31 & -0.64 & 5.67 \\
Pz-Pz & -5.01 & -1.92 & 3.09 & $2.71 / 458$ & 3.33 & -6.37 & -0.64 & 5.73 \\
\hline
\end{tabular}

${ }^{a}$ Obtained by DFT calculation (B3LYP, 6-31G*). ${ }^{b}$ HOMO-LUMO energy gap: $\mathrm{E}_{\text {gap }}=\mathrm{E}_{\mathrm{LUMO}}-\mathrm{E}_{\text {Hомо }}$.

${ }^{c}$ Vertical excitation of the lowest transition $\left(\mathrm{S}_{0} \rightarrow \mathrm{S}_{1}\right)$ calculated by TD-DFT (B3LYP, 6-311G**). d

Egap(opt): optical energy gap determined by onset of the absorption spectra. ${ }^{e}$ Energy levels determined by DFT calculations (CAM-B3LYP, 6-31G*). ${ }^{f}$ HOMO-LUMO energy gap: $\mathrm{E}_{\text {gap }}=\mathrm{E}_{\mathrm{LUMO}}-\mathrm{E}_{\text {Hомо }}$ (CAM-B3LYP, 6-31G*).

Table S5. Results of TD-DFT calculations (B3LYP, 6-311G**) for Cz-Cz.

\begin{tabular}{|c|c|c|c|c|}
\hline Compound & Transition & $\lambda, \mathrm{nm}(\mathrm{eV})$ & $\begin{array}{c}\text { Oscillator } \\
\text { Strength, } \\
f\end{array}$ & Orbital Contributions \\
\hline \multirow{6}{*}{$\mathbf{C z}-\mathbf{C z}$} & $\mathrm{S}_{0} \rightarrow \mathrm{S}_{1}$ & $401(3.10)$ & 0.0338 & $\mathrm{HOMO} \rightarrow$ LUMO $(98 \%)$ \\
\hline & $\mathrm{S}_{1} \rightarrow \mathrm{S}_{2}$ & $398(3.12)$ & 0.0516 & HOMO- $1 \rightarrow$ LUMO (99\%) \\
\hline & $\mathrm{S}_{1} \rightarrow \mathrm{S}_{3}$ & $350(3.54)$ & 0.0000 & HOMO-2 $\rightarrow$ LUMO (100\%) \\
\hline & $\mathrm{S}_{1} \rightarrow \mathrm{S}_{4}$ & $350(3.54)$ & 0.0000 & HOMO-3 $\rightarrow$ LUMO (30\%) \\
\hline & $\mathrm{S}_{1} \rightarrow \mathrm{S}_{5}$ & $341(3.63)$ & 0.0048 & $\begin{array}{l}\text { HOMO-6 } \rightarrow \text { LUMO }(28 \%) \\
\text { HOMO-5 } \rightarrow \text { LUMO }(65 \%)\end{array}$ \\
\hline & $\mathrm{S}_{0} \rightarrow \mathrm{S}_{6}$ & $319(3.88)$ & 0.2015 & $\begin{array}{l}\mathrm{HOMO}-1 \rightarrow \mathrm{LUMO}+1(27 \%) \\
\mathrm{HOMO} \rightarrow \mathrm{LUMO}+2(66 \%)\end{array}$ \\
\hline
\end{tabular}




\begin{tabular}{|c|c|c|c|l|}
\hline $\mathrm{S}_{0} \rightarrow \mathrm{S}_{7}$ & $318(3.90)$ & 0.0396 & $\begin{array}{l}\text { HOMO-1 } \rightarrow \text { LUMO+2 (54\%) } \\
\text { HOMO } \rightarrow \text { LUMO+1 (32\%) }\end{array}$ \\
\hline & $314(3.95)$ & 0.1170 & $\begin{array}{l}\text { HOMO-1 } \rightarrow \text { LUMO+3 (23\%) } \\
\text { HOMO-1 } \rightarrow \text { LUMO+5 (20\%) } \\
\text { HOMO } \rightarrow \text { LUMO+4 (46\%) }\end{array}$ \\
\hline $\mathrm{S}_{0} \rightarrow \mathrm{S}_{8}$ & $313(3.96)$ & 0.0637 & $\begin{array}{l}\text { HOMO-1 } \rightarrow \text { LUMO+4 (39\%) } \\
\text { HOMO } \rightarrow \text { LUMO+3 (25\%) }\end{array}$ \\
\hline $\mathrm{S}_{0} \rightarrow \mathrm{S}_{9}$ & $308(4.03)$ & 0.0544 & $\begin{array}{l}\text { HOMO-1 } \rightarrow \text { LUMO+3 (31\%) } \\
\text { HOMO-1 } \rightarrow \mathrm{LUMO}+5(45 \%)\end{array}$ \\
\hline
\end{tabular}

Table S6. Results of TD-DFT calculations (B3LYP, 6-311G**) for Cz-Npa.

\begin{tabular}{|c|c|c|c|c|}
\hline Compound & Transition & $\lambda, \mathbf{n m}(\mathrm{eV})$ & $\begin{array}{c}\text { Oscillator } \\
\text { Strength, } \\
f\end{array}$ & Orbital Contributions \\
\hline \multirow{9}{*}{ Cz-Npa } & $\mathrm{S}_{0} \rightarrow \mathrm{S}_{1}$ & $488(2.54)$ & 0.0000 & HOMO $\rightarrow$ LUMO (100\%) \\
\hline & $\mathrm{S}_{0} \rightarrow \mathrm{S}_{2}$ & $421(2.94)$ & 0.0000 & HOMO- $1 \rightarrow$ LUMO (100\%) \\
\hline & $\mathrm{S}_{0} \rightarrow \mathrm{S}_{3}$ & $393(3.16)$ & 0.0462 & $\mathrm{HOMO} \rightarrow$ LUMO+1 (98\%) \\
\hline & $\mathrm{S}_{0} \rightarrow \mathrm{S}_{4}$ & $378(3.28)$ & 0.0018 & HOMO-2 $\rightarrow$ LUMO $(91 \%)$ \\
\hline & $\mathrm{S}_{0} \rightarrow \mathrm{S}_{5}$ & $344(3.60)$ & 0.0000 & HOMO-1 $\rightarrow$ LUMO+1 (100\%) \\
\hline & $\mathrm{S}_{0} \rightarrow \mathrm{S}_{6}$ & $343(3.61)$ & 0.0230 & HOMO-3 $\rightarrow$ LUMO+1 (81\%) \\
\hline & $\mathrm{S}_{0} \rightarrow \mathrm{S}_{7}$ & $341(3.64)$ & 0.0055 & HOMO-3 $\rightarrow$ LUMO $(41 \%)$ \\
\hline & $\mathrm{S}_{0} \rightarrow \mathrm{S}_{8}$ & $339(3.66)$ & 0.0112 & HOMO-3 $\rightarrow$ LUMO $(55.3 \%)$ \\
\hline & $\mathrm{S}_{0} \rightarrow \mathrm{S}_{9}$ & $333(3.73)$ & 0.1089 & $\begin{array}{l}\text { HOMO-5 } \rightarrow \text { LUMO }(31 \%) \\
\text { HOMO-4 } \rightarrow \text { LUMO }(51 \%)\end{array}$ \\
\hline
\end{tabular}




\begin{tabular}{|l|l|l|l|l|}
\hline & $\mathrm{S}_{0} \rightarrow \mathrm{S}_{10}$ & $332(3.73)$ & 0.1591 & $\begin{array}{l}\text { HOMO-5 } \rightarrow \text { LUMO (60\%) } \\
\text { HOMO-4 } \rightarrow \text { LUMO (40\%) }\end{array}$ \\
\hline
\end{tabular}

Table S7. Results of TD-DFT calculations (B3LYP, 6-311G**) for Cz-Npa.

\begin{tabular}{|c|c|c|c|c|}
\hline Compound & Transition & $\lambda, \mathrm{nm}(\mathrm{eV})$ & $\begin{array}{c}\text { Oscillator } \\
\text { Strength, } \\
f\end{array}$ & Orbital Contributions \\
\hline \multirow{10}{*}{ Cz-NpaBr } & $\mathrm{S}_{0} \rightarrow \mathrm{S}_{1}$ & $523(2.37)$ & 0.0000 & $\mathrm{HOMO} \rightarrow$ LUMO $(100 \%)$ \\
\hline & $\mathrm{S}_{0} \rightarrow \mathrm{S}_{2}$ & $447(2.77)$ & 0.0000 & HOMO- $1 \rightarrow$ LUMO $(100 \%)$ \\
\hline & $\mathrm{S}_{0} \rightarrow \mathrm{S}_{3}$ & $394(3.14)$ & 0.0457 & $\mathrm{HOMO} \rightarrow \mathrm{LUMO}+1(98 \%)$ \\
\hline & $\mathrm{S}_{0} \rightarrow \mathrm{S}_{4}$ & $390(3.18)$ & 0.0029 & HOMO-2 $\rightarrow$ LUMO (92\%) \\
\hline & $\mathrm{S}_{0} \rightarrow \mathrm{S}_{5}$ & $354(3.50)$ & 0.0004 & HOMO-1 $\rightarrow$ LUMO+1 (95\%) \\
\hline & $\mathrm{S}_{0} \rightarrow \mathrm{S}_{6}$ & $349(3.56)$ & 0.1001 & $\begin{array}{l}\text { HOMO-5 } \rightarrow \text { LUMO }+1(25 \%) \\
\text { HOMO- } \rightarrow \text { LUMO+1 }(52 \%)\end{array}$ \\
\hline & $\mathrm{S}_{0} \rightarrow \mathrm{S}_{7}$ & $347(3.57)$ & 0.2204 & $\begin{array}{l}\text { HOMO-5 } \rightarrow \text { LUMO }(61 \%) \\
\text { HOMO-4 } \rightarrow \text { LUMO }(36 \%)\end{array}$ \\
\hline & $\mathrm{S}_{0} \rightarrow \mathrm{S}_{8}$ & $346(3.59)$ & 0.0000 & HOMO- $1 \rightarrow$ LUMO+1 (100\%) \\
\hline & $\mathrm{S}_{0} \rightarrow \mathrm{S}_{9}$ & $345(3.60)$ & 0.0389 & $\begin{array}{l}\text { HOMO-11 } \rightarrow \text { LUMO }(31 \%) \\
\text { HOMO-7 } \rightarrow \text { LUMO }(29 \%)\end{array}$ \\
\hline & $\mathrm{S}_{0} \rightarrow \mathrm{S}_{10}$ & $343(3.62)$ & 0.0020 & HOMO-3 $\rightarrow$ LUMO +1(85\%) \\
\hline
\end{tabular}

Table S8. Results of TD-DFT calculations (B3LYP, 6-311G**) for Cz-BMes. 


\begin{tabular}{|c|c|c|c|c|}
\hline Compound & Transition & $\lambda, \mathbf{n m}(\mathrm{eV})$ & $\begin{array}{c}\text { Oscillator } \\
\text { Strength, } \\
f \\
\end{array}$ & Orbital Contributions \\
\hline \multirow{10}{*}{ Cz-BMes } & $\mathrm{S}_{0} \rightarrow \mathrm{S}_{1}$ & $397(3.12)$ & 0.0437 & $\mathrm{HOMO} \rightarrow$ LUMO $(89 \%)$ \\
\hline & $\mathrm{S}_{0} \rightarrow \mathrm{S}_{2}$ & $371(3.35)$ & 0.0032 & $\mathrm{HOMO} \rightarrow \mathrm{LUMO}+1(88 \%)$ \\
\hline & $\mathrm{S}_{0} \rightarrow \mathrm{S}_{3}$ & $354(3.50)$ & 0.0685 & $\begin{array}{l}\text { HOMO-3 } \rightarrow \text { LUMO }(48 \%) \\
\text { HOMO-3 } \rightarrow \text { LUMO+1 }(49 \%)\end{array}$ \\
\hline & $\mathrm{S}_{0} \rightarrow \mathrm{S}_{4}$ & $351(3.53)$ & 0.4491 & HOMO-2 $\rightarrow$ LUMO $(63 \%)$ \\
\hline & $\mathrm{S}_{0} \rightarrow \mathrm{S}_{5}$ & $348(3.56)$ & 0.0006 & HOMO-1 $\rightarrow$ LUMO (92\%) \\
\hline & $\mathrm{S}_{0} \rightarrow \mathrm{S}_{6}$ & $345(3.60)$ & 0.0427 & $\begin{array}{l}\text { HOMO-7 } \rightarrow \text { LUMO }(24 \%) \\
\text { HOMO-2 } \rightarrow \text { LUMO+1 }(31 \%)\end{array}$ \\
\hline & $\mathrm{S}_{0} \rightarrow \mathrm{S}_{7}$ & $339(3.66)$ & 0.0408 & $\begin{array}{l}\text { HOMO-4 } \rightarrow \text { LUMO }(47 \%) \\
\text { HOMO-4 } \rightarrow \text { LUMO+1 }(43 \%)\end{array}$ \\
\hline & $\mathrm{S}_{0} \rightarrow \mathrm{S}_{8}$ & $331(3.75)$ & 0.0082 & $\begin{array}{l}\text { HOMO-5 } \rightarrow \text { LUMO }(49 \%) \\
\text { HOMO-5 } \rightarrow \text { LUMO+1 }(45 \%)\end{array}$ \\
\hline & $\mathrm{S}_{0} \rightarrow \mathrm{S}_{9}$ & $330(3.76)$ & 0.0000 & HOMO- $1 \rightarrow$ LUMO+1 $(92 \%)$ \\
\hline & $\mathrm{S}_{0} \rightarrow \mathrm{S}_{10}$ & $327(3.80)$ & 0.1190 & $\begin{array}{l}\text { HOMO }-6 \rightarrow \text { LUMO }(25 \%) \\
\text { HOMO-2 } \rightarrow \text { LUMO+1 }(20 \%)\end{array}$ \\
\hline
\end{tabular}

Table S9. Results of TD-DFT calculations (B3LYP, 6-311G**) for Pz-Npa.

\begin{tabular}{|c|c|c|c|l|}
\hline Compound & Transition & $\lambda, \mathbf{n m}(\mathbf{e V})$ & $\begin{array}{c}\text { Oscillator } \\
\text { Strength, } \\
f\end{array}$ & Orbital Contributions \\
\hline \multirow{2}{*}{ Pz-Npa } & $\mathrm{S}_{0} \rightarrow \mathrm{S}_{1}$ & $587(2.11)$ & 0.0000 & HOMO $\rightarrow$ LUMO (100\%) \\
\cline { 2 - 5 } & $\mathrm{S}_{0} \rightarrow \mathrm{S}_{2}$ & $455(2.73)$ & 0.0016 & HOMO $\rightarrow$ LUMO+1 (99\%) \\
\hline
\end{tabular}




\begin{tabular}{|c|c|c|c|l|}
\hline $\mathrm{S}_{0} \rightarrow \mathrm{S}_{3}$ & $390(3.18)$ & 0.0000 & HOMO-1 $\rightarrow$ LUMO (99\%) \\
\cline { 2 - 5 } & $\mathrm{S}_{0} \rightarrow \mathrm{S}_{4}$ & $377(3.29)$ & 0.0016 & HOMO-2 $\rightarrow$ LUMO (90\%) \\
\cline { 2 - 5 } & $\mathrm{S}_{0} \rightarrow \mathrm{S}_{5}$ & $360(3.44)$ & 0.0001 & $\begin{array}{l}\text { HOMO } \rightarrow \text { LUMO+5 (43\%) } \\
\text { HOMO } \rightarrow \text { LUMO+6 (44\%) }\end{array}$ \\
\hline $\mathrm{S}_{0} \rightarrow \mathrm{S}_{6}$ & $352(3.52)$ & 0.0005 & $\begin{array}{l}\text { HOMO } \rightarrow \text { LUMO+4 (31\%) } \\
\text { HOMO } \rightarrow \text { LUMO+6 (27\%) }\end{array}$ \\
\hline $\mathrm{S}_{0} \rightarrow \mathrm{S}_{7}$ & $344(3.60)$ & 0.0186 & HOMO-3 $\rightarrow$ LUMO+1 (86\%) \\
\cline { 2 - 5 } & $343(3.62)$ & 0.0013 & $\begin{array}{l}\text { HOMO } \rightarrow \text { LUMO+5 (49\%) } \\
\text { HOMO } \rightarrow \text { LUMO+7 (45\%) }\end{array}$ \\
\hline $\mathrm{S}_{0} \rightarrow \mathrm{S}_{8}$ & $34.67)$ & 0.0200 & $\begin{array}{l}\text { HOMO-8 } \rightarrow \text { LUMO (23\%) } \\
\text { HOMO-3 } \rightarrow \text { LUMO (31\%) }\end{array}$ \\
\hline $\mathrm{S}_{0} \rightarrow \mathrm{S}_{9}$ & $342(3.62)$ & 0.0020 & HOMO-3 $\rightarrow$ LUMO (62\%) \\
\hline
\end{tabular}

Table S10. Results of TD-DFT calculations (B3LYP, 6-311G**) for Pz-NpaBr.

\begin{tabular}{|c|l|l|l|l|}
\hline Compound & Transition & $\lambda, \mathbf{n m}(\mathbf{e V})$ & $\begin{array}{c}\text { Oscillator } \\
\text { Strength, } \\
\boldsymbol{f}\end{array}$ & \multicolumn{1}{|c|}{ Orbital Contributions } \\
\hline \multirow{4}{*}{ Pz-NpaBr } & $\mathrm{S}_{0} \rightarrow \mathrm{S}_{1}$ & $637(1.95)$ & 0.0000 & HOMO $\rightarrow$ LUMO (100\%) \\
\cline { 2 - 5 } & $\mathrm{S}_{0} \rightarrow \mathrm{S}_{2}$ & $457(2.72)$ & 0.0017 & HOMO $\rightarrow$ LUMO+1 (99\%) \\
\cline { 2 - 5 } & $\mathrm{S}_{0} \rightarrow \mathrm{S}_{3}$ & $412(3.01)$ & 0.0000 & HOMO-1 $\rightarrow$ LUMO (100\%) \\
\cline { 2 - 5 } & & & & \\
& $\mathrm{S}_{0} \rightarrow \mathrm{S}_{4}$ & $389(3.18)$ & 0.0026 & HOMO-2 $\rightarrow$ LUMO (91\%) \\
\hline
\end{tabular}




\begin{tabular}{|c|l|l|l|l|}
\hline $\mathrm{S}_{0} \rightarrow \mathrm{S}_{5}$ & $361(3.43)$ & 0.0001 & $\begin{array}{l}\text { HOMO } \rightarrow \text { LUMO+4 (45\%) } \\
\text { HOMO } \rightarrow \text { LUMO+5 (39\%) }\end{array}$ \\
\cline { 2 - 5 } & $\mathrm{S}_{0} \rightarrow \mathrm{S}_{6}$ & $356(3.48)$ & 0.0007 & HOMO-3 $\rightarrow$ LUMO (90\%) \\
\cline { 2 - 5 } & $\mathrm{S}_{0} \rightarrow \mathrm{S}_{7}$ & $354(3.50)$ & 0.0010 & $\begin{array}{l}\text { HOMO } \rightarrow \text { LUMO+3 (43\%) } \\
\text { HOMO } \rightarrow \text { LUMO+6 (27\%) }\end{array}$ \\
\hline $\mathrm{S}_{0} \rightarrow \mathrm{S}_{8}$ & $352(3.52)$ & 0.0000 & HOMO $\rightarrow$ LUMO+2 (96\%) \\
\cline { 2 - 5 } & $\mathrm{S}_{0} \rightarrow \mathrm{S}_{9}$ & $348(3.56)$ & 0.2594 & HOMO-5 $\rightarrow$ LUMO (66\%) \\
\cline { 2 - 5 } & $347(3.57)$ & 0.0002 & HOMO $\rightarrow$ LUMO+3 (47\%) \\
\hline
\end{tabular}

Table S11. Results of TD-DFT calculations (B3LYP, 6-311G**) for Pz-BMes.

\begin{tabular}{|c|c|c|c|c|}
\hline Compound & Transition & $\lambda, \mathbf{n m}(\mathrm{eV})$ & $\begin{array}{c}\text { Oscillator } \\
\text { Strength, } \\
f\end{array}$ & Orbital Contributions \\
\hline \multirow{5}{*}{ Pz-BMes } & $\mathrm{S}_{0} \rightarrow \mathrm{S}_{1}$ & $453(2.73)$ & 0.0017 & $\mathrm{HOMO} \rightarrow \mathrm{LUMO}(83 \%)$ \\
\hline & $\mathrm{S}_{0} \rightarrow \mathrm{S}_{2}$ & $427(2.90)$ & 0.0012 & $\mathrm{HOMO} \rightarrow \mathrm{LUMO}+1(82 \%)$ \\
\hline & $\mathrm{S}_{0} \rightarrow \mathrm{S}_{3}$ & $369(3.36)$ & 0.0001 & $\mathrm{HOMO} \rightarrow \mathrm{LUMO}+2(85 \%)$ \\
\hline & $\mathrm{S}_{0} \rightarrow \mathrm{S}_{4}$ & $358(3.46)$ & 0.0711 & $\begin{array}{l}\text { HOMO-3 } \rightarrow \text { LUMO }(23 \%) \\
\text { HOMO-3 } \rightarrow \text { LUMO+1 }(20 \%) \\
\text { HOMO-2 } \rightarrow \text { LUMO }(28 \%) \\
\text { HOMO-2 } \rightarrow \text { LUMO }+1(21 \%)\end{array}$ \\
\hline & $\mathrm{S}_{0} \rightarrow \mathrm{S}_{5}$ & $352(3.53)$ & 0.4308 & HOMO- $1 \rightarrow$ LUMO (41\%) \\
\hline
\end{tabular}




\begin{tabular}{|l|l|l|l|l|}
\hline & $\mathrm{S}_{0} \rightarrow \mathrm{S}_{6}$ & $346(3.59)$ & 0.0054 & HOMO-7 $\rightarrow$ LUMO (27\%) \\
\hline
\end{tabular}

Table S12. Results of TD-DFT calculations (B3LYP, 6-311G**) for Pz-Pz.

\begin{tabular}{|c|c|c|c|c|}
\hline Compound & Transition & $\lambda, \mathrm{nm}(\mathrm{eV})$ & $\begin{array}{c}\text { Oscillator } \\
\text { Strength, } \\
f\end{array}$ & Orbital Contributions \\
\hline \multirow{6}{*}{$\mathbf{P z}-\mathbf{P z}$} & $\mathrm{S}_{0} \rightarrow \mathrm{S}_{1}$ & $458(2.71)$ & 0.0016 & $\mathrm{HOMO} \rightarrow$ LUMO $(98 \%)$ \\
\hline & $\mathrm{S}_{0} \rightarrow \mathrm{S}_{2}$ & $458(2.71)$ & 0.0011 & HOMO- $1 \rightarrow$ LUMO (98\%) \\
\hline & $\mathrm{S}_{0} \rightarrow \mathrm{S}_{3}$ & $374(3.32)$ & 0.0006 & $\mathrm{HOMO} \rightarrow \mathrm{LUMO}+1(71 \%)$ \\
\hline & $\mathrm{S}_{0} \rightarrow \mathrm{S}_{4}$ & $374(3.32)$ & 0.0001 & HOMO- $1 \rightarrow$ LUMO+1 (71\%) \\
\hline & $\mathrm{S}_{0} \rightarrow \mathrm{S}_{5}$ & $346(3.58)$ & 0.0001 & $\mathrm{HOMO} \rightarrow \mathrm{LUMO}+3(81 \%)$ \\
\hline & $\mathrm{S}_{0} \rightarrow \mathrm{S}_{6}$ & $346(3.58)$ & 0.0000 & HOMO $-1 \rightarrow$ LUMO $+3(80 \%)$ \\
\hline
\end{tabular}

Table S13. Coordinates $(\AA)$ for the optimized structure (B3LYP, 6-31G*) of Cz-Cz.

\begin{tabular}{|r|r|r|r|r|r|r|r|}
\hline Atom & $\mathrm{X}$ & $\mathrm{Y}$ & $\mathrm{Z}$ & Atom & $\mathrm{X}$ & $\mathrm{Y}$ & $\mathrm{Z}$ \\
\hline $\mathrm{C}$ & -0.50051 & 1.226432 & 1.208267 & $\mathrm{C}$ & 8.139517 & 0.154786 & -1.32011 \\
\hline $\mathrm{C}$ & -1.40205 & 0.537746 & 0.180548 & $\mathrm{C}$ & 7.995488 & -1.15351 & -0.86768 \\
\hline $\mathrm{C}$ & -0.86154 & 0.535017 & -1.11777 & $\mathrm{C}$ & 6.774122 & -1.55324 & -0.3128 \\
\hline $\mathrm{C}$ & 0.500508 & 1.226432 & -1.20827 & $\mathrm{C}$ & 5.707716 & -0.62231 & -0.2279 \\
\hline $\mathrm{C}$ & -0.27644 & 2.633533 & 0.663653 & $\mathrm{C}$ & 5.850461 & 0.696605 & -0.66755 \\
\hline $\mathrm{C}$ & 0.276437 & 2.633533 & -0.66365 & $\mathrm{C}$ & 6.29472 & -2.79473 & 0.258195 \\
\hline $\mathrm{C}$ & 1.402052 & 0.537747 & -0.18055 & $\mathrm{C}$ & 4.956767 & -2.56887 & 0.670669 \\
\hline $\mathrm{C}$ & 0.861545 & 0.535018 & 1.117767 & $\mathrm{C}$ & 6.868859 & -4.05817 & 0.441236 \\
\hline $\mathrm{C}$ & 1.564265 & -0.05547 & 2.161562 & $\mathrm{C}$ & 6.109951 & -5.07098 & 1.020411 \\
\hline $\mathrm{C}$ & 2.811211 & -0.63838 & 1.914339 & $\mathrm{C}$ & 4.780985 & -4.83339 & 1.411065 \\
\hline $\mathrm{C}$ & 3.345138 & -0.64345 & 0.620693 & $\mathrm{C}$ & 4.185379 & -3.58526 & 1.240812 \\
\hline
\end{tabular}




\begin{tabular}{|r|r|r|r|r|r|r|r|}
\hline $\mathrm{C}$ & 2.632211 & -0.05213 & -0.43645 & $\mathrm{H}$ & -0.91787 & 1.24479 & 2.21631 \\
\hline $\mathrm{C}$ & -1.56426 & -0.05547 & -2.16156 & $\mathrm{H}$ & 0.917872 & 1.24479 & -2.21631 \\
\hline $\mathrm{C}$ & -2.81121 & -0.63838 & -1.91434 & $\mathrm{H}$ & 1.157628 & -0.05389 & 3.169488 \\
\hline $\mathrm{C}$ & -3.34514 & -0.64345 & -0.62069 & $\mathrm{H}$ & 3.380535 & -1.07988 & 2.725922 \\
\hline $\mathrm{C}$ & -2.63221 & -0.05213 & 0.436454 & $\mathrm{H}$ & 3.044588 & -0.07226 & -1.44082 \\
\hline $\mathrm{N}$ & -0.55251 & 3.725896 & 1.322684 & $\mathrm{H}$ & -1.15763 & -0.05389 & -3.16949 \\
\hline $\mathrm{C}$ & -0.27622 & 4.900162 & 0.659556 & $\mathrm{H}$ & -3.38053 & -1.07988 & -2.72592 \\
\hline $\mathrm{N}$ & 0.552507 & 3.725896 & -1.32268 & $\mathrm{H}$ & -3.04459 & -0.07226 & 1.440819 \\
\hline $\mathrm{C}$ & 0.276223 & 4.900162 & -0.65956 & $\mathrm{H}$ & -0.963 & 6.106292 & 2.299927 \\
\hline $\mathrm{C}$ & -0.54412 & 6.132831 & 1.298599 & $\mathrm{H}$ & -0.48069 & 8.266827 & 1.14632 \\
\hline $\mathrm{C}$ & -0.27353 & 7.321664 & 0.652216 & $\mathrm{H}$ & 0.480691 & 8.266827 & -1.14632 \\
\hline $\mathrm{C}$ & 0.273523 & 7.321664 & -0.65222 & $\mathrm{H}$ & 0.963 & 6.106292 & -2.29993 \\
\hline $\mathrm{C}$ & 0.544116 & 6.132831 & -1.2986 & $\mathrm{H}$ & -7.21093 & 2.088156 & 1.565386 \\
\hline $\mathrm{N}$ & -4.60631 & -1.24603 & -0.3719 & $\mathrm{H}$ & -9.08225 & 0.475919 & 1.753826 \\
\hline $\mathrm{C}$ & -7.07651 & 1.068223 & 1.215469 & $\mathrm{H}$ & -8.82187 & -1.85588 & 0.939635 \\
\hline $\mathrm{C}$ & -8.13952 & 0.154787 & 1.320101 & $\mathrm{H}$ & -5.03886 & 1.411387 & 0.580427 \\
\hline $\mathrm{C}$ & -7.99549 & -1.15351 & 0.86768 & $\mathrm{H}$ & -7.89333 & -4.24593 & -0.13031 \\
\hline $\mathrm{C}$ & -6.77412 & -1.55324 & 0.312795 & $\mathrm{H}$ & -6.54519 & -6.05508 & -1.16895 \\
\hline $\mathrm{C}$ & -5.70772 & -0.62231 & 0.2279 & $\mathrm{H}$ & -4.20129 & -5.63906 & -1.85345 \\
\hline $\mathrm{C}$ & -5.85046 & 0.696605 & 0.667551 & $\mathrm{H}$ & -3.15573 & -3.41315 & -1.53638 \\
\hline $\mathrm{C}$ & -6.29472 & -2.79473 & -0.2582 & $\mathrm{H}$ & 7.210929 & 2.088156 & -1.56539 \\
\hline $\mathrm{C}$ & -4.95677 & -2.56888 & -0.67067 & $\mathrm{H}$ & 9.082247 & 0.475918 & -1.75383 \\
\hline $\mathrm{C}$ & -6.86886 & -4.05817 & -0.44124 & $\mathrm{H}$ & 8.821865 & -1.85588 & -0.93964 \\
\hline $\mathrm{C}$ & -6.10995 & -5.07098 & -1.02041 & $\mathrm{H}$ & 5.038862 & 1.411388 & -0.58043 \\
\hline $\mathrm{C}$ & -4.78098 & -4.83339 & -1.41106 & $\mathrm{H}$ & 7.893326 & -4.24593 & 0.130302 \\
\hline $\mathrm{C}$ & -4.18538 & -3.58526 & -1.24081 & $\mathrm{H}$ & 6.545194 & -6.05508 & 1.168948 \\
\hline $\mathrm{C}$ & 4.606309 & -1.24603 & 0.371902 & $\mathrm{H}$ & 4.201287 & -5.63906 & 1.853456 \\
\hline $\mathrm{C}$ & 7.076506 & 1.068223 & -1.21547 & $\mathrm{H}$ & 3.155734 & -3.41315 & 1.536388 \\
\hline
\end{tabular}

Table S14. Coordinates ( $⿱$ ) for the optimized structure (B3LYP, 6-31G*) of Cz-Npa.

\begin{tabular}{|r|r|r|r|r|r|r|r|}
\hline Atom & $\mathrm{X}$ & $\mathrm{Y}$ & $\mathrm{Z}$ & Atom & $\mathrm{X}$ & $\mathrm{Y}$ & $\mathrm{Z}$ \\
\hline $\mathrm{C}$ & 1.081787 & 1.39785 & -1.09925 & $\mathrm{C}$ & -9.11193 & -0.00398 & -0.94593 \\
\hline $\mathrm{C}$ & 1.905162 & 0.508149 & -0.16494 & $\mathrm{C}$ & -9.33581 & -1.21021 & -0.31391 \\
\hline $\mathrm{C}$ & 1.369424 & 0.419806 & 1.132446 & $\mathrm{C}$ & -8.25135 & -2.00365 & 0.144121 \\
\hline $\mathrm{C}$ & 0.090379 & 1.239446 & 1.315653 & $\mathrm{C}$ & -6.92099 & -1.52606 & -0.06325 \\
\hline $\mathrm{C}$ & 1.014407 & 2.75214 & -0.40021 & $\mathrm{C}$ & -6.71466 & -0.28455 & -0.71545 \\
\hline $\mathrm{C}$ & 0.467178 & 2.665306 & 0.927104 & $\mathrm{C}$ & -8.42993 & -3.25033 & 0.799172 \\
\hline $\mathrm{C}$ & -0.88119 & 0.7695 & 0.230595 & $\mathrm{C}$ & -7.34373 & -3.98641 & 1.227023 \\
\hline $\mathrm{C}$ & -0.34761 & 0.852295 & -1.06732 & $\mathrm{C}$ & -6.03251 & -3.50962 & 1.020564 \\
\hline $\mathrm{C}$ & -1.11017 & 0.463909 & -2.16229 & $\mathrm{C}$ & -5.82018 & -2.29817 & 0.38586 \\
\hline $\mathrm{C}$ & -2.40793 & -0.01632 & -1.96176 & $\mathrm{C}$ & -4.42971 & -1.81969 & 0.184515 \\
\hline
\end{tabular}




\begin{tabular}{|r|r|r|r|r|r|r|r|}
\hline $\mathrm{C}$ & -2.93279 & -0.08937 & -0.67333 & $\mathrm{C}$ & -5.34162 & 0.231702 & -0.94151 \\
\hline $\mathrm{C}$ & -2.17401 & 0.307559 & 0.432804 & $\mathrm{O}$ & -5.12061 & 1.29349 & -1.49987 \\
\hline $\mathrm{C}$ & 2.00689 & -0.3529 & 2.095996 & $\mathrm{O}$ & -3.4549 & -2.45002 & 0.560779 \\
\hline $\mathrm{C}$ & 3.184993 & -1.03265 & 1.770665 & $\mathrm{H}$ & 1.497854 & 1.482463 & -2.10445 \\
\hline $\mathrm{C}$ & 3.714007 & -0.94969 & 0.477925 & $\mathrm{H}$ & -0.32133 & 1.191039 & 2.324991 \\
\hline $\mathrm{C}$ & 3.06601 & -0.17538 & -0.49926 & $\mathrm{H}$ & -0.70499 & 0.530461 & -3.16836 \\
\hline $\mathrm{C}$ & 1.411647 & 3.873301 & -0.93756 & $\mathrm{H}$ & -3.01548 & -0.32342 & -2.80631 \\
\hline $\mathrm{C}$ & 1.268499 & 4.989804 & -0.14506 & $\mathrm{H}$ & -2.5954 & 0.243695 & 1.431037 \\
\hline $\mathrm{N}$ & 0.316871 & 3.701303 & 1.706757 & $\mathrm{H}$ & 1.602821 & -0.42076 & 3.102631 \\
\hline $\mathrm{N}$ & 4.90755 & -1.64641 & 0.149215 & $\mathrm{H}$ & 3.704857 & -1.6196 & 2.521112 \\
\hline $\mathrm{C}$ & 0.720727 & 4.904201 & 1.172931 & $\mathrm{H}$ & 3.473252 & -0.12819 & -1.50481 \\
\hline $\mathrm{C}$ & 1.672655 & 6.248401 & -0.64716 & $\mathrm{H}$ & 2.085152 & 6.286688 & -1.65079 \\
\hline $\mathrm{C}$ & 1.537038 & 7.380861 & 0.129738 & $\mathrm{H}$ & 1.848163 & 8.346239 & -0.25979 \\
\hline $\mathrm{C}$ & 0.993843 & 7.296465 & 1.432972 & $\mathrm{H}$ & 0.893372 & 8.19793 & 2.031123 \\
\hline $\mathrm{C}$ & 0.592407 & 6.080422 & 1.947294 & $\mathrm{H}$ & 0.17302 & 5.989309 & 2.944633 \\
\hline $\mathrm{C}$ & 7.6221 & 0.578694 & -1.136 & $\mathrm{H}$ & 7.864276 & 1.614511 & -1.35805 \\
\hline $\mathrm{C}$ & 8.588658 & -0.41916 & -1.34978 & $\mathrm{H}$ & 9.565392 & -0.14556 & -1.73864 \\
\hline $\mathrm{C}$ & 8.306057 & -1.75139 & -1.06255 & $\mathrm{H}$ & 9.057767 & -2.52075 & -1.21928 \\
\hline $\mathrm{C}$ & 7.042524 & -2.08881 & -0.56338 & $\mathrm{H}$ & 5.619788 & 1.048332 & -0.46973 \\
\hline $\mathrm{C}$ & 6.074912 & -1.07066 & -0.36637 & $\mathrm{H}$ & 7.874686 & -4.91681 & -0.45115 \\
\hline $\mathrm{C}$ & 6.356734 & 0.270565 & -0.64084 & $\mathrm{H}$ & 6.33271 & -6.69017 & 0.353602 \\
\hline $\mathrm{C}$ & 6.430901 & -3.33478 & -0.15048 & $\mathrm{H}$ & 4.033331 & -6.1207 & 1.078132 \\
\hline $\mathrm{C}$ & 5.116846 & -3.02412 & 0.283608 & $\mathrm{H}$ & 3.225839 & -3.77616 & 1.034466 \\
\hline $\mathrm{C}$ & 6.870051 & -4.66368 & -0.12224 & $\mathrm{H}$ & -7.60766 & 1.409016 & -1.64396 \\
\hline $\mathrm{C}$ & 6.002784 & -5.65553 & 0.326285 & $\mathrm{H}$ & -9.9503 & 0.593605 & -1.2914 \\
\hline $\mathrm{C}$ & 4.699175 & -5.33126 & 0.740001 & $\mathrm{H}$ & -10.3504 & -1.56894 & -0.15855 \\
\hline $\mathrm{C}$ & 4.236876 & -4.017 & 0.723002 & $\mathrm{H}$ & -9.44038 & -3.61836 & 0.959416 \\
\hline $\mathrm{N}$ & -4.27904 & -0.57653 & -0.47261 & $\mathrm{H}$ & -7.49405 & -4.93886 & 1.726601 \\
\hline & -7.79637 & 0.462357 & -1.14857 & $\mathrm{H}$ & -5.17062 & -4.07867 & 1.352982 \\
\hline & & & & & & \\
\hline
\end{tabular}

Table S15. Coordinates $(\AA)$ for the optimized structure (B3LYP, 6-31G*) of CzNpaBr.

\begin{tabular}{|r|r|r|r|r|r|r|r|}
\hline Atom & $\mathrm{X}$ & $\mathrm{Y}$ & $\mathrm{Z}$ & Atom & $\mathrm{X}$ & $\mathrm{Y}$ & $\mathrm{Z}$ \\
\hline $\mathrm{C}$ & 2.274082 & 1.482989 & -1.09224 & $\mathrm{C}$ & -8.00671 & 1.66189 & -1.09212 \\
\hline $\mathrm{C}$ & 2.927051 & 0.460398 & -0.15864 & $\mathrm{C}$ & -8.43273 & 0.456951 & -0.57292 \\
\hline $\mathrm{C}$ & 2.346012 & 0.427766 & 1.121672 & $\mathrm{C}$ & -7.49891 & -0.53771 & -0.18281 \\
\hline $\mathrm{C}$ & 1.198484 & 1.425411 & 1.290915 & $\mathrm{C}$ & -6.10204 & -0.26157 & -0.34006 \\
\hline $\mathrm{C}$ & 2.38459 & 2.818612 & -0.36348 & $\mathrm{C}$ & -5.69307 & 0.985629 & -0.87832 \\
\hline $\mathrm{C}$ & 1.79295 & 2.787131 & 0.946844 & $\mathrm{C}$ & -7.85139 & -1.80629 & 0.360674 \\
\hline $\mathrm{C}$ & 0.201183 & 1.125853 & 0.169331 & $\mathrm{C}$ & -6.89884 & -2.73558 & 0.72288 \\
\hline $\mathrm{C}$ & 0.779354 & 1.155611 & -1.11191 & $\mathrm{C}$ & -5.5325 & -2.44066 & 0.560753 \\
\hline
\end{tabular}




\begin{tabular}{|c|c|c|c|c|c|c|c|}
\hline $\mathrm{C}$ & 0.00177 & 0.906853 & -2.23656 & $\mathrm{C}$ & -5.13309 & -1.22444 & 0.038848 \\
\hline $\mathrm{C}$ & -1.35796 & 0.619214 & -2.08288 & $\mathrm{C}$ & -3.68249 & -0.94967 & -0.11302 \\
\hline $\mathrm{C}$ & -1.92448 & 0.595316 & -0.81078 & $\mathrm{C}$ & -4.25184 & 1.301127 & -1.05541 \\
\hline $\mathrm{C}$ & -1.15053 & 0.852844 & 0.325287 & $\mathrm{O}$ & -3.86407 & 2.360593 & -1.51828 \\
\hline $\mathrm{C}$ & 2.828317 & -0.45403 & 2.081657 & $\mathrm{O}$ & -2.82413 & -1.75352 & 0.212158 \\
\hline $\mathrm{C}$ & 3.895857 & -1.30126 & 1.769011 & $\mathrm{H}$ & 2.727868 & 1.525551 & -2.08365 \\
\hline $\mathrm{C}$ & 4.468068 & -1.27518 & 0.492342 & $\mathrm{H}$ & 0.753926 & 1.418207 & 2.287359 \\
\hline $\mathrm{C}$ & 3.977605 & -0.38778 & -0.48075 & $\mathrm{H}$ & 0.44284 & 0.933299 & -3.22926 \\
\hline $\mathrm{N}$ & 2.952835 & 3.88093 & -0.86573 & $\mathrm{H}$ & -1.97905 & 0.421403 & -2.95025 \\
\hline $\mathrm{C}$ & 2.949789 & 4.991156 & -0.05189 & $\mathrm{H}$ & -1.60766 & 0.830636 & 1.309727 \\
\hline $\mathrm{N}$ & 1.77332 & 3.818271 & 1.746833 & $\mathrm{H}$ & 2.389032 & -0.48008 & 3.075413 \\
\hline $\mathrm{N}$ & 5.543793 & -2.14708 & 0.176442 & $\mathrm{H}$ & 4.296531 & -1.97822 & 2.516684 \\
\hline $\mathrm{C}$ & 2.361505 & 4.959899 & 1.251156 & $\mathrm{H}$ & 4.41591 & -0.38347 & -1.47429 \\
\hline $\mathrm{C}$ & 3.541789 & 6.188474 & -0.51622 & $\mathrm{H}$ & 3.981094 & 6.186737 & -1.50913 \\
\hline $\mathrm{C}$ & 3.549226 & 7.313422 & 0.283047 & $\mathrm{H}$ & 4.004407 & 8.231734 & -0.0775 \\
\hline $\mathrm{C}$ & 2.967346 & 7.282475 & 1.571918 & $\mathrm{H}$ & 2.981767 & 8.177341 & 2.187942 \\
\hline $\mathrm{C}$ & 2.383611 & 6.126826 & 2.04947 & $\mathrm{H}$ & 1.930199 & 6.07754 & 3.034772 \\
\hline $\mathrm{C}$ & 8.610684 & -0.36656 & -1.00521 & $\mathrm{H}$ & 9.020825 & 0.620301 & -1.20197 \\
\hline $\mathrm{C}$ & 9.41004 & -1.50315 & -1.21675 & $\mathrm{H}$ & 10.42722 & -1.38399 & -1.57895 \\
\hline $\mathrm{C}$ & 8.910935 & -2.77702 & -0.9611 & $\mathrm{H}$ & 9.53371 & -3.65436 & -1.11606 \\
\hline $\mathrm{C}$ & 7.597801 & -2.91515 & -0.49631 & $\mathrm{H}$ & 6.693262 & 0.407547 & -0.37365 \\
\hline $\mathrm{C}$ & 6.800905 & -1.75808 & -0.30158 & $\mathrm{H}$ & 7.96386 & -5.84177 & -0.41934 \\
\hline $\mathrm{C}$ & 7.30046 & -0.47545 & -0.54387 & $\mathrm{H}$ & 6.138489 & -7.35672 & 0.31632 \\
\hline $\mathrm{C}$ & 6.78478 & -4.05324 & -0.12112 & $\mathrm{H}$ & 3.941858 & -6.43729 & 0.99917 \\
\hline $\mathrm{C}$ & 5.526784 & -3.54274 & 0.289238 & $\mathrm{H}$ & 3.521303 & -3.99331 & 0.982818 \\
\hline $\mathrm{C}$ & 7.004754 & -5.43563 & -0.10839 & $\mathrm{H}$ & -6.28639 & 2.874439 & -1.65559 \\
\hline $\mathrm{C}$ & 5.978916 & -6.2823 & 0.301298 & $\mathrm{H}$ & -8.73616 & 2.411071 & -1.38468 \\
\hline $\mathrm{C}$ & 4.733744 & -5.75973 & 0.691425 & $\mathrm{H}$ & -9.49152 & 0.254305 & -0.45585 \\
\hline $\mathrm{C}$ & 4.488341 & -4.38826 & 0.689349 & $\mathrm{H}$ & -7.2044 & -3.69163 & 1.132649 \\
\hline $\mathrm{N}$ & -3.33292 & 0.304579 & -0.65776 & $\mathrm{H}$ & -4.77422 & -3.16308 & 0.843558 \\
\hline $\mathrm{C}$ & -6.63273 & 1.930772 & -1.24759 & $\mathrm{Br}$ & -9.68865 & -2.26316 & 0.608086 \\
\hline
\end{tabular}

Table S16. Coordinates $(\AA ̊)$ for the optimized structure (B3LYP, 6-31G*) of Cz-BMes.

\begin{tabular}{|r|r|r|r|r|r|r|r|}
\hline Atom & $\mathrm{X}$ & $\mathrm{Y}$ & $\mathrm{Z}$ & Atom & $\mathrm{X}$ & $\mathrm{Y}$ & $\mathrm{Z}$ \\
\hline $\mathrm{C}$ & 3.491876 & 1.188285 & -1.3916 & $\mathrm{H}$ & 7.444213 & -7.1239 & 1.84443 \\
\hline $\mathrm{C}$ & 4.157343 & 0.390348 & -0.26775 & $\mathrm{H}$ & 5.260145 & -6.08428 & 2.377695 \\
\hline $\mathrm{C}$ & 3.592531 & 0.636046 & 0.996661 & $\mathrm{H}$ & 4.814747 & -3.70471 & 1.840154 \\
\hline $\mathrm{C}$ & 2.447013 & 1.649638 & 0.961458 & $\mathrm{C}$ & -2.18679 & 0.172213 & -0.63411 \\
\hline $\mathrm{C}$ & 3.613834 & 2.649097 & -0.96905 & $\mathrm{C}$ & -2.8464 & -0.92755 & -1.21178 \\
\hline $\mathrm{C}$ & 3.038357 & 2.90286 & 0.324565 & $\mathrm{C}$ & -2.94401 & 1.049089 & 0.162995 \\
\hline $\mathrm{C}$ & 1.432676 & 1.123338 & -0.05773 & $\mathrm{C}$ & -4.20851 & -1.12724 & -1.0142 \\
\hline
\end{tabular}




\begin{tabular}{|c|c|c|c|c|c|c|c|}
\hline $\mathrm{C}$ & 1.997299 & 0.86976 & -1.32003 & $\mathrm{H}$ & -2.27842 & -1.63964 & -1.80448 \\
\hline $\mathrm{C}$ & 1.199651 & 0.383128 & -2.34894 & $\mathrm{C}$ & -4.30094 & 0.829466 & 0.374892 \\
\hline $\mathrm{C}$ & -0.15876 & 0.150523 & -2.11651 & $\mathrm{H}$ & -2.46668 & 1.923543 & 0.597061 \\
\hline $\mathrm{C}$ & -0.73957 & 0.406045 & -0.86374 & $\mathrm{C}$ & -4.98183 & -0.25893 & -0.21294 \\
\hline $\mathrm{C}$ & 0.082355 & 0.900161 & 0.169525 & $\mathrm{H}$ & -4.69 & -1.98201 & -1.48163 \\
\hline $\mathrm{C}$ & 4.092856 & -0.01445 & 2.118534 & $\mathrm{H}$ & -4.85713 & 1.525018 & 0.997526 \\
\hline $\mathrm{C}$ & 5.161355 & -0.90677 & 1.983457 & $\mathrm{~B}$ & -6.5232 & -0.4816 & 0.005024 \\
\hline $\mathrm{C}$ & 5.716873 & -1.15795 & 0.723866 & $\mathrm{C}$ & -7.22522 & 0.208004 & 1.248591 \\
\hline $\mathrm{C}$ & 5.207527 & -0.50563 & -0.41195 & $\mathrm{C}$ & -8.28578 & 1.139539 & 1.052276 \\
\hline $\mathrm{N}$ & 4.178044 & 3.57616 & -1.6943 & $\mathrm{C}$ & -6.80467 & -0.03681 & 2.580008 \\
\hline $\mathrm{C}$ & 4.188573 & 4.83528 & -1.1377 & $\mathrm{C}$ & -8.86124 & 1.790781 & 2.143311 \\
\hline $\mathrm{N}$ & 3.030007 & 4.082306 & 0.883654 & $\mathrm{C}$ & -7.42127 & 0.626897 & 3.650809 \\
\hline $\mathrm{C}$ & 3.61574 & 5.087765 & 0.147944 & $\mathrm{C}$ & -8.44653 & 1.548312 & 3.458322 \\
\hline $\mathrm{C}$ & 4.779899 & 5.901314 & -1.85425 & $\mathrm{H}$ & -9.65841 & 2.510607 & 1.964118 \\
\hline $\mathrm{C}$ & 4.802121 & 7.17129 & -1.31467 & $\mathrm{H}$ & -7.08528 & 0.410341 & 4.664035 \\
\hline $\mathrm{C}$ & 4.235516 & 7.421039 & -0.04296 & $\mathrm{C}$ & -7.32179 & -1.38048 & -1.02831 \\
\hline $\mathrm{C}$ & 3.652111 & 6.398505 & 0.677171 & $\mathrm{C}$ & -8.0107 & -2.54799 & -0.5875 \\
\hline $\mathrm{N}$ & 6.795103 & -2.07209 & 0.588294 & $\mathrm{C}$ & -7.35144 & -1.08578 & -2.41494 \\
\hline $\mathrm{C}$ & 9.811261 & -0.58561 & -1.04561 & $\mathrm{C}$ & -8.66379 & -3.36907 & -1.50649 \\
\hline $\mathrm{C}$ & 10.61413 & -1.73912 & -1.02827 & $\mathrm{C}$ & -8.03351 & -1.92973 & -3.30391 \\
\hline $\mathrm{C}$ & 10.13378 & -2.92744 & -0.48585 & $\mathrm{C}$ & -8.69195 & -3.07856 & -2.87577 \\
\hline $\mathrm{C}$ & 8.835792 & -2.96329 & 0.037024 & $\mathrm{H}$ & -9.16768 & -4.26432 & -1.14538 \\
\hline $\mathrm{C}$ & 8.034438 & -1.79387 & -0.00166 & $\mathrm{H}$ & -8.04803 & -1.67266 & -4.36225 \\
\hline $\mathrm{C}$ & 8.515923 & -0.5938 & -0.53241 & $\mathrm{C}$ & -6.73964 & 0.168582 & -3.0106 \\
\hline $\mathrm{C}$ & 8.04444 & -3.99389 & 0.676216 & $\mathrm{H}$ & -6.16316 & -0.06666 & -3.91325 \\
\hline $\mathrm{C}$ & 6.794149 & -3.40966 & 1.00324 & $\mathrm{H}$ & -7.52629 & 0.873708 & -3.31082 \\
\hline $\mathrm{C}$ & 8.278561 & -5.33928 & 0.983564 & $\mathrm{H}$ & -6.0753 & 0.691361 & -2.32147 \\
\hline $\mathrm{C}$ & 7.273706 & -6.07936 & 1.599726 & $\mathrm{C}$ & -8.03229 & -2.96304 & 0.870158 \\
\hline $\mathrm{C}$ & 6.035653 & -5.48796 & 1.90452 & $\mathrm{H}$ & -8.60338 & -3.8879 & 0.999802 \\
\hline $\mathrm{C}$ & 5.776608 & -4.1509 & 1.610244 & $\mathrm{H}$ & -7.02354 & -3.1463 & 1.259845 \\
\hline $\mathrm{H}$ & 3.932301 & 1.013534 & -2.37455 & $\mathrm{H}$ & -8.48585 & -2.19479 & 1.504465 \\
\hline $\mathrm{H}$ & 2.016456 & 1.859926 & 1.941891 & $\mathrm{C}$ & -8.8007 & 1.486516 & -0.33054 \\
\hline $\mathrm{H}$ & 1.624313 & 0.19266 & -3.33139 & $\mathrm{H}$ & -8.01285 & 1.905822 & -0.96812 \\
\hline $\mathrm{H}$ & -0.78417 & -0.20565 & -2.92975 & $\mathrm{H}$ & -9.19839 & 0.608236 & -0.84916 \\
\hline $\mathrm{H}$ & -0.34011 & 1.083092 & 1.153835 & $\mathrm{H}$ & -9.60002 & 2.232042 & -0.26929 \\
\hline $\mathrm{H}$ & 3.668456 & 0.177024 & 3.100668 & $\mathrm{C}$ & -5.74702 & -1.06408 & 2.938138 \\
\hline $\mathrm{H}$ & 5.576634 & -1.40113 & 2.855801 & $\mathrm{H}$ & -6.20912 & -1.9472 & 3.399294 \\
\hline $\mathrm{H}$ & 5.633188 & -0.71566 & -1.38859 & $\mathrm{H}$ & -5.17337 & -1.40639 & 2.075628 \\
\hline $\mathrm{H}$ & 5.207589 & 5.683872 & -2.82825 & $\mathrm{H}$ & -5.03762 & -0.65853 & 3.669339 \\
\hline $\mathrm{H}$ & 5.257397 & 7.987815 & -1.86826 & $\mathrm{C}$ & -9.09086 & 2.268875 & 4.618943 \\
\hline $\mathrm{H}$ & 4.261489 & 8.426814 & 0.367164 & $\mathrm{H}$ & -10.1786 & 2.126118 & 4.624828 \\
\hline $\mathrm{H}$ & 3.210867 & 6.564447 & 1.655251 & $\mathrm{H}$ & -8.70078 & 1.912476 & 5.577655 \\
\hline
\end{tabular}




\begin{tabular}{|r|r|r|r|r|r|r|r|}
\hline $\mathrm{H}$ & 10.20705 & 0.335494 & -1.46484 & $\mathrm{H}$ & -8.91133 & 3.350521 & 4.567496 \\
\hline $\mathrm{H}$ & 11.61937 & -1.69944 & -1.43805 & $\mathrm{C}$ & -9.4152 & -3.98403 & -3.84448 \\
\hline $\mathrm{H}$ & 10.75914 & -3.81622 & -0.46358 & $\mathrm{H}$ & -9.00053 & -4.99993 & -3.82863 \\
\hline $\mathrm{H}$ & 7.907206 & 0.304327 & -0.54068 & $\mathrm{H}$ & -10.4797 & -4.07032 & -3.5924 \\
\hline $\mathrm{H}$ & 9.232339 & -5.79996 & 0.739521 & $\mathrm{H}$ & -9.34435 & -3.61043 & -4.87096 \\
\hline
\end{tabular}

Table S17. Coordinates $(\AA)$ for the optimized structure (B3LYP, 6-31G*) of Pz-Npa.

\begin{tabular}{|c|c|c|c|c|c|c|c|}
\hline Atom & $X$ & $\mathrm{Y}$ & $\mathrm{Z}$ & Atom & $X$ & $\mathrm{Y}$ & $\mathrm{Z}$ \\
\hline $\mathrm{C}$ & 0.817773 & 1.405615 & -1.20073 & $\mathrm{C}$ & -9.35527 & -0.06193 & -0.72828 \\
\hline $\mathrm{C}$ & 1.662243 & 0.595384 & -0.21401 & $\mathrm{C}$ & -9.55324 & -1.23395 & -0.02708 \\
\hline $\mathrm{C}$ & 1.148501 & 0.60443 & 1.095795 & $\mathrm{C}$ & -8.45119 & -1.99419 & 0.445059 \\
\hline $\mathrm{C}$ & -0.13738 & 1.422349 & 1.233898 & $\mathrm{C}$ & -7.13031 & -1.5194 & 0.178839 \\
\hline $\mathrm{C}$ & 0.744951 & 2.808711 & -0.60643 & $\mathrm{C}$ & -6.95076 & -0.31309 & -0.54374 \\
\hline $\mathrm{C}$ & 0.218638 & 2.818022 & 0.731861 & $\mathrm{C}$ & -8.60333 & -3.20517 & 1.170019 \\
\hline $\mathrm{C}$ & -1.11837 & 0.860208 & 0.202257 & $\mathrm{C}$ & -7.50098 & -3.91009 & 1.608852 \\
\hline $\mathrm{C}$ & -0.60484 & 0.85018 & -1.10639 & $\mathrm{C}$ & -6.19917 & -3.4364 & 1.344017 \\
\hline $\mathrm{C}$ & -1.3785 & 0.372362 & -2.15745 & $\mathrm{C}$ & -6.01248 & -2.25915 & 0.640592 \\
\hline $\mathrm{C}$ & -2.66726 & -0.10549 & -1.90121 & $\mathrm{C}$ & -4.63155 & -1.78267 & 0.379064 \\
\hline $\mathrm{C}$ & -3.17165 & -0.08816 & -0.60278 & $\mathrm{C}$ & -5.58813 & 0.199982 & -0.83095 \\
\hline 6 & -2.40188 & 0.399276 & 0.458693 & $\mathrm{O}$ & -5.38832 & 1.231525 & -1.45063 \\
\hline $\mathrm{C}$ & 1.808076 & -0.07992 & 2.109583 & $\mathrm{O}$ & -3.64167 & -2.38509 & 0.761534 \\
\hline $\mathrm{C}$ & 2.983243 & -0.7816 & 1.816102 & $\mathrm{H}$ & 1.219059 & 1.417598 & -2.21542 \\
\hline $\mathrm{C}$ & 3.494737 & -0.79292 & 0.517464 & $\mathrm{H}$ & -0.53433 & 1.446903 & 2.249929 \\
\hline $\mathrm{C}$ & 2.830183 & -0.09699 & -0.50677 & $\mathrm{H}$ & -0.98908 & 0.368309 & -3.17192 \\
\hline $\mathrm{N}$ & 1.120392 & 3.88976 & -1.23415 & $\mathrm{H}$ & -3.28393 & -0.48175 & -2.71053 \\
\hline $\mathrm{C}$ & 0.975974 & 5.061931 & -0.52695 & $\mathrm{H}$ & -2.80833 & 0.405751 & 1.465191 \\
\hline $\mathrm{N}$ & 0.069042 & 3.908618 & 1.433137 & $\mathrm{H}$ & 1.416311 & -0.07343 & 3.123251 \\
\hline $\mathrm{C}$ & 0.450524 & 5.071422 & 0.802954 & $\mathrm{H}$ & 3.512501 & -1.32597 & 2.591869 \\
\hline $\mathrm{C}$ & 1.35617 & 6.28304 & -1.13028 & $\mathrm{H}$ & 3.234977 & -0.10816 & -1.51518 \\
\hline $\mathrm{C}$ & 1.21902 & 7.469764 & -0.43942 & $\mathrm{H}$ & 1.751796 & 6.249541 & -2.14082 \\
\hline $\mathrm{C}$ & 0.698541 & 7.479189 & 0.875784 & $\mathrm{H}$ & 1.511621 & 8.406024 & -0.90662 \\
\hline $\mathrm{C}$ & 0.320634 & 6.301648 & 1.488007 & $\mathrm{H}$ & 0.596983 & 8.422557 & 1.405133 \\
\hline $\mathrm{C}$ & 4.39179 & -5.44468 & -1.36113 & $\mathrm{H}$ & -0.08128 & 6.282063 & 2.496429 \\
\hline $\mathrm{C}$ & 5.595634 & -4.99158 & -0.82127 & $\mathrm{H}$ & 4.322565 & -6.44646 & -1.77456 \\
\hline $\mathrm{C}$ & 5.689258 & -3.71625 & -0.26277 & $\mathrm{H}$ & 6.470599 & -5.63566 & -0.80824 \\
\hline $\mathrm{C}$ & 4.587262 & -2.83856 & -0.28413 & $\mathrm{H}$ & 2.514074 & -2.66063 & -0.8531 \\
\hline $\mathrm{C}$ & 3.384417 & -3.3049 & -0.83792 & $\mathrm{H}$ & 2.337094 & -4.92962 & -1.76534 \\
\hline $\mathrm{C}$ & 3.286816 & -4.59674 & -1.35586 & $\mathrm{H}$ & 9.295755 & -1.37518 & 0.202833 \\
\hline$S$ & 7.179949 & -3.25109 & 0.592808 & $\mathrm{H}$ & 9.333317 & 1.108341 & 0.014498 \\
\hline $\mathrm{C}$ & 7.157379 & -1.50185 & 0.261361 & $\mathrm{H}$ & 7.168737 & 2.354824 & -0.04427 \\
\hline $\mathrm{C}$ & 5.929544 & -0.81361 & 0.19421 & $\mathrm{H}$ & 5.038276 & 1.145212 & 0.043585 \\
\hline
\end{tabular}




\begin{tabular}{|r|r|r|r|r|r|r|r|}
\hline $\mathrm{N}$ & 4.702852 & -1.52861 & 0.255133 & $\mathrm{H}$ & -7.88061 & 1.321895 & -1.53877 \\
\hline $\mathrm{C}$ & 8.367024 & -0.81225 & 0.169352 & $\mathrm{H}$ & -10.2069 & 0.510402 & -1.08391 \\
\hline $\mathrm{C}$ & 8.386667 & 0.578673 & 0.065231 & $\mathrm{H}$ & -10.5606 & -1.5908 & 0.173066 \\
\hline $\mathrm{C}$ & 7.178629 & 1.271039 & 0.031843 & $\mathrm{H}$ & -9.60648 & -3.57078 & 1.375302 \\
\hline $\mathrm{C}$ & 5.964502 & 0.585322 & 0.080948 & $\mathrm{H}$ & -7.63119 & -4.83537 & 2.1622 \\
\hline $\mathrm{N}$ & -4.50831 & -0.57433 & -0.34493 & $\mathrm{H}$ & -5.32482 & -3.98118 & 1.684511 \\
\hline $\mathrm{C}$ & -8.04906 & 0.401885 & -0.98885 & & & & \\
\hline
\end{tabular}

Table S18. Coordinates (Å) for the optimized structure (B3LYP, 6-31G*) of Pz-NpaBr.

\begin{tabular}{|c|c|c|c|c|c|c|c|}
\hline Atom & $\mathrm{X}$ & $Y$ & $\mathrm{Z}$ & Atom & $X$ & $Y$ & $\mathrm{Z}$ \\
\hline $\mathrm{C}$ & -1.99171 & 1.536519 & 1.198757 & $\mathrm{C}$ & 8.290227 & 1.584773 & 1.011141 \\
\hline $\mathrm{C}$ & -2.67043 & 0.580615 & 0.214261 & $\mathrm{C}$ & 8.688922 & 0.396749 & 0.434407 \\
\hline $\mathrm{C}$ & -2.11286 & 0.623552 & -1.07677 & $\mathrm{C}$ & 7.733623 & -0.56685 & 0.019198 \\
\hline $\mathrm{C}$ & -0.95385 & 1.614936 & -1.20044 & $\mathrm{C}$ & 6.34399 & -0.27738 & 0.212191 \\
\hline $\mathrm{C}$ & -2.09652 & 2.916119 & 0.556825 & $\mathrm{C}$ & 5.963283 & 0.951951 & 0.809362 \\
\hline $\mathrm{C}$ & -1.52562 & 2.9596 & -0.76211 & $\mathrm{C}$ & 8.057781 & -1.81636 & -0.58314 \\
\hline $\mathrm{C}$ & 0.056291 & 1.232599 & -0.11568 & $\mathrm{C}$ & 7.085323 & -2.71566 & -0.9675 \\
\hline $\mathrm{C}$ & -0.50124 & 1.191344 & 1.174444 & $\mathrm{C}$ & 5.726539 & -2.40828 & -0.76913 \\
\hline $\mathrm{C}$ & 0.290512 & 0.866847 & 2.269491 & $\mathrm{C}$ & 5.354381 & -1.20958 & -0.18966 \\
\hline $\mathrm{C}$ & 1.643699 & 0.572353 & 2.076325 & $\mathrm{C}$ & 3.911129 & -0.92117 & 0.000318 \\
\hline $\mathrm{C}$ & 2.189331 & 0.616328 & 0.795679 & $\mathrm{C}$ & 4.530345 & 1.280387 & 1.025113 \\
\hline $\mathrm{C}$ & 1.401411 & 0.9515 & -0.31011 & $\mathrm{O}$ & 4.165692 & 2.324543 & 1.538807 \\
\hline $\mathrm{C}$ & -2.62489 & -0.18267 & -2.0863 & $\mathrm{O}$ & 3.034583 & -1.69843 & -0.34054 \\
\hline $\mathrm{C}$ & -3.69466 & -1.04081 & -1.80666 & $\mathrm{H}$ & -2.43017 & 1.522172 & 2.197873 \\
\hline $\mathrm{C}$ & -4.2488 & -1.0869 & -0.52632 & $\mathrm{H}$ & -0.52466 & 1.665234 & -2.20231 \\
\hline $\mathrm{C}$ & -3.73393 & -0.26804 & 0.493283 & $\mathrm{H}$ & -0.13449 & 0.840308 & 3.269201 \\
\hline $\mathrm{N}$ & -2.64443 & 3.951308 & 1.132937 & $\mathrm{H}$ & 2.276138 & 0.315479 & 2.919741 \\
\hline $\mathrm{C}$ & -2.64026 & 5.109866 & 0.389775 & $\mathrm{H}$ & 1.842727 & 0.982435 & -1.30151 \\
\hline $\mathrm{N}$ & -1.50591 & 4.038141 & -1.49691 & $\mathrm{H}$ & -2.19915 & -0.15046 & -3.08569 \\
\hline $\mathrm{C}$ & -2.07229 & 5.153237 & -0.92204 & $\mathrm{H}$ & -4.10828 & -1.68145 & -2.57913 \\
\hline $\mathrm{C}$ & -3.21109 & 6.282307 & 0.93677 & $\mathrm{H}$ & -4.17128 & -0.30772 & 1.487273 \\
\hline $\mathrm{C}$ & -3.21743 & 7.454544 & 0.208736 & $\mathrm{H}$ & -3.63531 & 6.223741 & 1.934478 \\
\hline $\mathrm{C}$ & -2.65564 & 7.497459 & -1.08877 & $\mathrm{H}$ & -3.65622 & 8.353613 & 0.632534 \\
\hline $\mathrm{C}$ & -2.09266 & 6.367634 & -1.64622 & $\mathrm{H}$ & -2.66908 & 8.428978 & -1.64782 \\
\hline $\mathrm{C}$ & -4.5268 & -5.76587 & 1.475897 & $\mathrm{H}$ & -1.6548 & 6.374684 & -2.6397 \\
\hline $\mathrm{C}$ & -5.76002 & -5.51237 & 0.875156 & $\mathrm{H}$ & -4.32768 & -6.73483 & 1.923954 \\
\hline $\mathrm{C}$ & -6.01744 & -4.28042 & 0.272414 & $\mathrm{H}$ & -6.52845 & -6.28006 & 0.849397 \\
\hline $\mathrm{C}$ & -5.05942 & -3.24794 & 0.307804 & $\mathrm{H}$ & -3.06266 & -2.74624 & 0.952008 \\
\hline $\mathrm{C}$ & -3.82611 & -3.51393 & 0.923512 & $\mathrm{H}$ & -2.59084 & -4.93904 & 1.943511 \\
\hline $\mathrm{C}$ & -3.56061 & -4.76264 & 1.486482 & $\mathrm{H}$ & -9.90748 & -2.51576 & -0.39435 \\
\hline$S$ & -7.52286 & -4.06392 & -0.65348 & $\mathrm{H}$ & -10.3199 & -0.06173 & -0.27991 \\
\hline
\end{tabular}




\begin{tabular}{|r|r|r|r|r|r|r|r|}
\hline $\mathrm{C}$ & -7.77337 & -2.32325 & -0.37386 & $\mathrm{H}$ & -8.36774 & 1.49454 & -0.17971 \\
\hline $\mathrm{C}$ & -6.66476 & -1.4584 & -0.28279 & $\mathrm{H}$ & -6.07947 & 0.613966 & -0.15674 \\
\hline $\mathrm{N}$ & -5.34471 & -1.98505 & -0.2789 & $\mathrm{H}$ & 6.598391 & 2.797367 & 1.654941 \\
\hline $\mathrm{C}$ & -9.07452 & -1.81987 & -0.34467 & $\mathrm{H}$ & 9.035897 & 2.310138 & 1.322203 \\
\hline $\mathrm{C}$ & -9.30395 & -0.44539 & -0.28158 & $\mathrm{H}$ & 9.742346 & 0.183911 & 0.29035 \\
\hline $\mathrm{C}$ & -8.21399 & 0.419941 & -0.22511 & $\mathrm{H}$ & 7.369405 & -3.65772 & -1.42258 \\
\hline $\mathrm{C}$ & -6.91071 & -0.07809 & -0.21132 & $\mathrm{H}$ & 4.952866 & -3.10728 & -1.06867 \\
\hline $\mathrm{N}$ & 3.590351 & 0.314395 & 0.602558 & $\mathrm{Br}$ & 9.883401 & -2.28855 & -0.88172 \\
\hline $\mathrm{C}$ & 6.923346 & 1.866876 & 1.201609 & & & & \\
\hline
\end{tabular}

Table S19. Coordinates ( $\AA$ ) for the optimized structure (B3LYP, 6-31G*) of Pz-BMes.

\begin{tabular}{|r|r|r|r|r|r|r|r|}
\hline Atom & $\mathrm{X}$ & $\mathrm{Y}$ & $\mathrm{Z}$ & Atom & $\mathrm{X}$ & $\mathrm{Y}$ & $\mathrm{Z}$ \\
\hline $\mathrm{C}$ & 3.181283 & 1.443823 & -1.398692 & $\mathrm{H}$ & 11.52839 & -0.26651 & 1.294951 \\
\hline $\mathrm{C}$ & 3.874659 & 0.557045 & -0.36161 & $\mathrm{H}$ & 9.556434 & 0.957438 & 2.218989 \\
\hline $\mathrm{C}$ & 3.327447 & 0.678814 & 0.92835 & $\mathrm{H}$ & 7.279117 & 0.183407 & 1.738098 \\
\hline $\mathrm{C}$ & 2.164561 & 1.670054 & 1.000685 & $\mathrm{C}$ & -2.47028 & 0.274766 & -0.65549 \\
\hline $\mathrm{C}$ & 3.284005 & 2.862393 & -0.84697 & $\mathrm{C}$ & -3.44334 & 0.634634 & -1.6047 \\
\hline $\mathrm{C}$ & 2.725163 & 2.986606 & 0.472311 & $\mathrm{C}$ & -2.90496 & -0.35312 & 0.525992 \\
\hline $\mathrm{C}$ & 1.14557 & 1.214423 & -0.04754 & $\mathrm{C}$ & -4.79084 & 0.371645 & -1.38087 \\
\hline $\mathrm{C}$ & 1.692341 & 1.096693 & -1.3373 & $\mathrm{H}$ & -3.13893 & 1.146479 & -2.51367 \\
\hline $\mathrm{C}$ & 0.88181 & 0.711777 & -2.39879 & $\mathrm{C}$ & -4.25659 & -0.58884 & 0.753302 \\
\hline $\mathrm{C}$ & -0.47053 & 0.44201 & -2.17068 & $\mathrm{H}$ & -2.17183 & -0.67389 & 1.261332 \\
\hline $\mathrm{C}$ & -1.03184 & 0.554826 & -0.88836 & $\mathrm{C}$ & -5.24503 & -0.23681 & -0.19093 \\
\hline $\mathrm{C}$ & -0.1973 & 0.948956 & 0.177366 & $\mathrm{H}$ & -5.51686 & 0.658386 & -2.13696 \\
\hline $\mathrm{C}$ & 3.853882 & -0.05824 & 1.983324 & $\mathrm{H}$ & -4.56021 & -1.06934 & 1.679965 \\
\hline $\mathrm{C}$ & 4.927978 & -0.92447 & 1.749871 & $\mathrm{~B}$ & -6.77205 & -0.49907 & 0.070602 \\
\hline $\mathrm{C}$ & 5.473374 & -1.04611 & 0.467204 & $\mathrm{C}$ & -7.27691 & -0.65509 & 1.565768 \\
\hline $\mathrm{C}$ & 4.944189 & -0.29877 & -0.59457 & $\mathrm{C}$ & -7.02697 & 0.348689 & 2.539166 \\
\hline $\mathrm{N}$ & 3.818375 & 3.862568 & -1.49328 & $\mathrm{C}$ & -7.98166 & -1.81811 & 1.982187 \\
\hline $\mathrm{C}$ & 3.814625 & 5.065833 & -0.82483 & $\mathrm{C}$ & -7.48041 & 0.184215 & 3.853468 \\
\hline $\mathrm{N}$ & 2.705177 & 4.1096 & 1.137243 & $\mathrm{C}$ & -8.39029 & -1.95643 & 3.311214 \\
\hline $\mathrm{C}$ & 3.259463 & 5.189194 & 0.487318 & $\mathrm{C}$ & -8.15894 & -0.96255 & 4.265778 \\
\hline $\mathrm{C}$ & 4.373717 & 6.204036 & -1.45057 & $\mathrm{H}$ & -7.29375 & 0.97766 & 4.575724 \\
\hline $\mathrm{C}$ & 4.381218 & 7.419969 & -0.79812 & $\mathrm{H}$ & -8.91151 & -2.8649 & 3.608543 \\
\hline $\mathrm{C}$ & 3.831799 & 7.5421 & 0.499617 & $\mathrm{C}$ & -7.75525 & -0.57815 & -1.1713 \\
\hline $\mathrm{C}$ & 3.280097 & 6.44711 & 1.132898 & $\mathrm{C}$ & -7.57393 & -1.52921 & -2.20764 \\
\hline $\mathrm{C}$ & 5.805751 & -6.05641 & -0.42393 & $\mathrm{C}$ & -8.8345 & 0.343082 & -1.30297 \\
\hline $\mathrm{C}$ & 7.036357 & -5.50166 & -0.77571 & $\mathrm{C}$ & -8.43392 & -1.54272 & -3.3149 \\
\hline $\mathrm{C}$ & 7.279473 & -4.13924 & -0.59678 & $\mathrm{C}$ & -9.65881 & 0.304946 & -2.42837 \\
\hline $\mathrm{C}$ & 6.308747 & -3.30655 & -0.0057 & $\mathrm{C}$ & -9.48135 & -0.63522 & -3.44955 \\
\hline $\mathrm{C}$ & 5.078661 & -3.88029 & 0.352755 & $\mathrm{H}$ & -8.27537 & -2.28959 & -4.09179 \\
\hline & & & & & &
\end{tabular}




\begin{tabular}{|r|r|r|r|r|r|r|r|}
\hline $\mathrm{C}$ & 4.827799 & -5.23492 & 0.131815 & $\mathrm{H}$ & -10.4643 & 1.032731 & -2.51329 \\
\hline $\mathrm{S}$ & 8.784554 & -3.4351 & -1.23711 & $\mathrm{C}$ & -8.28417 & -2.95694 & 1.028609 \\
\hline $\mathrm{C}$ & 9.012064 & -2.13151 & -0.04547 & $\mathrm{H}$ & -8.80177 & -3.76941 & 1.548578 \\
\hline $\mathrm{C}$ & 7.892328 & -1.47074 & 0.497206 & $\mathrm{H}$ & -8.91678 & -2.63152 & 0.196706 \\
\hline $\mathrm{N}$ & 6.578937 & -1.92686 & 0.201729 & $\mathrm{H}$ & -7.37155 & -3.37892 & 0.590996 \\
\hline $\mathrm{C}$ & 10.30656 & -1.71344 & 0.265649 & $\mathrm{C}$ & -6.28955 & 1.638361 & 2.223366 \\
\hline $\mathrm{C}$ & 10.51768 & -0.59724 & 1.075182 & $\mathrm{H}$ & -6.62175 & 2.441341 & 2.89037 \\
\hline $\mathrm{C}$ & 9.416453 & 0.082824 & 1.589737 & $\mathrm{H}$ & -5.20725 & 1.526077 & 2.360132 \\
\hline $\mathrm{C}$ & 8.119799 & -0.3549 & 1.318012 & $\mathrm{H}$ & -6.44135 & 1.976643 & 1.195462 \\
\hline $\mathrm{H}$ & 3.610728 & 1.367205 & -2.39878 & $\mathrm{C}$ & -8.65432 & -1.11375 & 5.684619 \\
\hline $\mathrm{H}$ & 1.744982 & 1.783033 & 2.001704 & $\mathrm{H}$ & -8.08168 & -0.48905 & 6.378292 \\
\hline $\mathrm{H}$ & 1.295267 & 0.613781 & -3.39937 & $\mathrm{H}$ & -9.70829 & -0.81681 & 5.772255 \\
\hline $\mathrm{H}$ & -1.09429 & 0.115381 & -2.99749 & $\mathrm{H}$ & -8.58374 & -2.15271 & 6.025496 \\
\hline $\mathrm{H}$ & -0.61577 & 1.07299 & 1.172432 & $\mathrm{C}$ & -6.52166 & -2.62073 & -2.15287 \\
\hline $\mathrm{H}$ & 3.437076 & 0.035973 & 2.982739 & $\mathrm{H}$ & -5.742 & -2.42807 & -1.41422 \\
\hline $\mathrm{H}$ & 5.34857 & -1.5084 & 2.563273 & $\mathrm{H}$ & -6.98148 & -3.58633 & -1.90257 \\
\hline $\mathrm{H}$ & 5.38096 & -0.40131 & -1.5837 & $\mathrm{H}$ & -6.03425 & -2.74449 & -3.12706 \\
\hline $\mathrm{H}$ & 4.789261 & 6.084142 & -2.44643 & $\mathrm{C}$ & -9.10091 & 1.419016 & -0.26952 \\
\hline $\mathrm{H}$ & 4.811632 & 8.292356 & -1.28198 & $\mathrm{H}$ & -9.22401 & 1.00139 & 0.734077 \\
\hline $\mathrm{H}$ & 3.845822 & 8.507046 & 0.998765 & $\mathrm{H}$ & -8.27995 & 2.145601 & -0.21723 \\
\hline $\mathrm{H}$ & 2.852005 & 6.514986 & 2.128379 & $\mathrm{H}$ & -10.0091 & 1.977692 & -0.51726 \\
\hline $\mathrm{H}$ & 5.618117 & -7.11366 & -0.58595 & $\mathrm{C}$ & -10.4077 & -0.66981 & -4.64206 \\
\hline $\mathrm{H}$ & 7.814101 & -6.12044 & -1.21473 & $\mathrm{H}$ & -11.3907 & -1.07779 & -4.37156 \\
\hline $\mathrm{H}$ & 4.307079 & -3.26289 & 0.796067 & $\mathrm{H}$ & -10.5789 & 0.334748 & -5.04656 \\
\hline $\mathrm{H}$ & 3.860485 & -5.64417 & 0.409938 & $\mathrm{H}$ & -10.0028 & -1.29337 & -5.44576 \\
\hline $\mathrm{H}$ & 11.14868 & -2.25853 & -0.1517 & & & & \\
\hline
\end{tabular}

Table S20. Coordinates ( $\AA$ ) for the optimized structure (B3LYP, 6-31G*) of Pz-Pz.

\begin{tabular}{|r|r|r|r|r|r|r|r|}
\hline Atom & $\mathrm{X}$ & $\mathrm{Y}$ & $\mathrm{Z}$ & Atom & $\mathrm{X}$ & $\mathrm{Y}$ & $\mathrm{Z}$ \\
\hline $\mathrm{C}$ & -0.4589 & 1.367731 & 1.224806 & $\mathrm{C}$ & 5.768392 & -3.1163 & -0.64995 \\
\hline $\mathrm{C}$ & -1.39282 & 0.6757 & 0.228492 & $\mathrm{C}$ & 4.673628 & -2.52219 & 0.008517 \\
\hline $\mathrm{C}$ & -0.90008 & 0.677652 & -1.08858 & $\mathrm{C}$ & 3.646873 & -3.36143 & 0.468876 \\
\hline $\mathrm{C}$ & 0.458999 & 1.367561 & -1.22476 & $\mathrm{C}$ & 3.712107 & -4.74265 & 0.282141 \\
\hline $\mathrm{C}$ & -0.2526 & 2.774858 & 0.6727 & $\mathrm{~S}$ & 7.006898 & -2.09217 & -1.41722 \\
\hline $\mathrm{C}$ & 0.252809 & 2.774764 & -0.67281 & $\mathrm{C}$ & 7.007857 & -0.73994 & -0.25831 \\
\hline $\mathrm{C}$ & 1.392878 & 0.675581 & -0.22837 & $\mathrm{C}$ & 5.807417 & -0.35039 & 0.367473 \\
\hline $\mathrm{C}$ & 0.900133 & 0.677715 & 1.088701 & $\mathrm{~N}$ & 4.622311 & -1.11235 & 0.180156 \\
\hline $\mathrm{C}$ & 1.639055 & 0.096114 & 2.112799 & $\mathrm{C}$ & 8.184384 & -0.01787 & -0.05444 \\
\hline $\mathrm{C}$ & 2.872515 & -0.49705 & 1.820087 & $\mathrm{C}$ & 8.182648 & 1.136424 & 0.728847 \\
\hline $\mathrm{C}$ & 3.360985 & -0.50563 & 0.508976 & $\mathrm{C}$ & 6.993478 & 1.547085 & 1.326756 \\
\hline $\mathrm{C}$ & 2.617508 & 0.08825 & -0.52102 & $\mathrm{C}$ & 5.822524 & 0.806467 & 1.162222 \\
\hline
\end{tabular}




\begin{tabular}{|r|r|r|r|r|r|r|r|}
\hline $\mathrm{C}$ & -1.63904 & 0.09597 & -2.1126 & $\mathrm{H}$ & -0.84282 & 1.386159 & 2.245944 \\
\hline $\mathrm{C}$ & -2.87252 & -0.49711 & -1.81982 & $\mathrm{H}$ & 0.842924 & 1.385848 & -2.2459 \\
\hline $\mathrm{C}$ & -3.36098 & -0.50555 & -0.50869 & $\mathrm{H}$ & 1.264884 & 0.100633 & 3.133191 \\
\hline $\mathrm{C}$ & -2.61747 & 0.088443 & 0.521217 & $\mathrm{H}$ & 3.460782 & -0.95631 & 2.609066 \\
\hline $\mathrm{N}$ & -0.50407 & 3.867104 & 1.341631 & $\mathrm{H}$ & 3.012645 & 0.078474 & -1.53264 \\
\hline $\mathrm{C}$ & -0.25206 & 5.041151 & 0.668994 & $\mathrm{H}$ & -1.26489 & 0.100373 & -3.133 \\
\hline $\mathrm{N}$ & 0.504371 & 3.866916 & -1.34186 & $\mathrm{H}$ & -3.46082 & -0.95639 & -2.60875 \\
\hline $\mathrm{C}$ & 0.252453 & 5.041058 & -0.66935 & $\mathrm{H}$ & -3.0126 & 0.078791 & 1.532842 \\
\hline $\mathrm{C}$ & -0.49721 & 6.273861 & 1.317187 & $\mathrm{H}$ & -0.88067 & 6.247385 & 2.332578 \\
\hline $\mathrm{C}$ & -0.24988 & 7.462437 & 0.661274 & $\mathrm{H}$ & -0.4395 & 8.407693 & 1.16218 \\
\hline $\mathrm{C}$ & 0.250485 & 7.462344 & -0.66189 & $\mathrm{H}$ & 0.440186 & 8.407531 & -1.16289 \\
\hline $\mathrm{C}$ & 0.497714 & 6.273678 & -1.31767 & $\mathrm{H}$ & 0.881175 & 6.24706 & -2.33306 \\
\hline $\mathrm{C}$ & -4.81495 & -5.32325 & 0.340296 & $\mathrm{H}$ & -4.87496 & -6.39907 & 0.475339 \\
\hline $\mathrm{C}$ & -5.84672 & -4.50185 & 0.794886 & $\mathrm{H}$ & -6.71497 & -4.93002 & 1.288111 \\
\hline $\mathrm{C}$ & -5.76865 & -3.11608 & 0.649937 & $\mathrm{H}$ & -2.78535 & -2.93332 & -0.96597 \\
\hline $\mathrm{C}$ & -4.67384 & -2.52206 & -0.00853 & $\mathrm{H}$ & -2.89579 & -5.36238 & -0.64184 \\
\hline $\mathrm{C}$ & -3.64731 & -3.36139 & -0.4692 & $\mathrm{H}$ & -9.09844 & -0.35628 & 0.534329 \\
\hline $\mathrm{C}$ & -3.71282 & -4.74264 & -0.28277 & $\mathrm{H}$ & -9.09826 & 1.704292 & -0.86486 \\
\hline $\mathrm{S}$ & -7.0068 & -2.09178 & 1.41756 & $\mathrm{H}$ & -6.96807 & 2.444891 & -1.93832 \\
\hline $\mathrm{C}$ & -7.00783 & -0.73958 & 0.258605 & $\mathrm{H}$ & -4.91146 & 1.137921 & -1.64553 \\
\hline $\mathrm{C}$ & -5.80737 & -0.35012 & -0.36719 & $\mathrm{H}$ & 4.873978 & -6.39914 & -0.47626 \\
\hline $\mathrm{N}$ & -4.6223 & -1.11216 & -0.17975 & $\mathrm{H}$ & 6.714356 & -4.93029 & -1.2885 \\
\hline $\mathrm{C}$ & -8.18433 & -0.01749 & 0.054684 & $\mathrm{H}$ & 2.784962 & -2.93328 & 0.965668 \\
\hline $\mathrm{C}$ & -8.18253 & 1.136774 & -0.72864 & $\mathrm{H}$ & 2.89492 & -5.3623 & 0.641013 \\
\hline $\mathrm{C}$ & -6.99332 & 1.54738 & -1.32653 & $\mathrm{H}$ & 9.098473 & -0.3567 & -0.5341 \\
\hline $\mathrm{C}$ & -5.8224 & 0.806729 & -1.16196 & $\mathrm{H}$ & 9.098418 & 1.703901 & 0.865016 \\
\hline $\mathrm{C}$ & 4.814173 & -5.32335 & -0.34095 & $\mathrm{H}$ & 6.968255 & 2.444604 & 1.938539 \\
\hline $\mathrm{C}$ & 5.846169 & -4.50205 & -0.79523 & $\mathrm{H}$ & 4.911613 & 1.1377 & 1.645819 \\
\hline & & & & & & & \\
\hline
\end{tabular}

\section{References:}

[1] M. J. Frisch, G. W. Trucks, H. B. Schlegel, G. E. Scuseria, M. A. Robb, J. R. Cheeseman, G. Scalmani, V. Barone, B. Mennucci, G. A. H. Petersson, M. Nakatsuji, X. Caricato, H. P. F. Li, A. Hratchian, J. Izmaylov, G. Bloino, J. L. Zheng, M. Sonnenberg, M. Hada, K. Ehara, R. Toyota, J. Fukuda, M. Hasegawa, T. Ishida, Y. Nakajima, O. Honda, H. Kitao, T. Nakai, J. A. Vreven, J. E. Montgomery Jr., F. Peralta, M. Ogliaro, J. J. Bearpark, E. Heyd, K. N. Brothers, V. N. Kudin, T. Staroverov, R. Keith, J. Kobayashi, K. Normand, A. Raghavachari, J. C. Rendell, S. S. Burant, J. Iyengar, M. Tomasi, N. Cossi, J. M. Rega, M. Millam, J. E. Klene, J. B. Knox, V. Cross, C. Bakken, J. Adamo, R. Jaramillo, R. E. Gomperts, O. Stratmann, A. J. Yazyev, R. 
Austin, C. Cammi, J. W. Pomelli, R. L. Ochterski, K. Martin, V. G. Morokuma, G. A. Zakrzewski, P. Voth, J. J. Salvador, S. Dannenberg, A. D. Dapprich, O. Daniels, J. B. Farkas, J. V. Foresman, J. Ortiz, J. Cioslowski, D. J. Fox, Gaussian 09 Revision C.01, 2010.

[2] (a) C. Lee, W. Yang, R. G. Parr, Development of the Colle-Salvetti correlationenergy formula into a functional of the electron density. Phys. Rev. B, 1988, 37, 785789; (b) A. D. Becke, A new mixing of Hartree-Fock and local density-functional theories. J. Chem. Phys. 1993, 98, 1372-1377; (c) A. D. Becke, Density-functional thermochemistry. III. The role of exact exchange. J. Chem. Phys. 1993, 98, 5648-5652. [3] (a) R. Ditchfield, W. J. Hehre, J. A. Pople, Self-Consistent Molecular-Orbital Methods. IX. An Extended Gaussian-Type Basis for Molecular-Orbital Studies of Organic Molecules. J. Chem. Phys. 1971, 54, 724-728; (b) A. D. McLean, G. S. Chandler, Contracted Gaussian basis sets for molecular calculations. I. Second row atoms, $Z=11-18$. J. Chem. Phys. 1980, 72, 5639-5648. 